COMMUNICATIONS IN

ANALYSIS AND GEOMETRY

Volume 11, Number 1, 121-180, 2003

\title{
Rigidity and Vanishing Theorems in $K$-Theory
}

\author{
Kefeng LiU, ${ }^{1}$ Xiaonan Ma, ${ }^{2}$ and Weiping Zhang ${ }^{3}$
}

In this paper, we first establish a $K$-theory version of the equivariant family index theorem for a circle action, then use it to prove several family rigidity and vanishing theorems on the equivariant $K$-theory level.

\section{Introduction.}

In [W], Witten considered the indices of elliptic operators on the free loop space $\mathcal{L} M$ of a spin manifold $M$. In particular the index of the formal signature operator on the loop space is exactly the elliptic genus of LandweberStong-Ochanine $[\mathbf{L S}],[\mathbf{O}]$. Motivated by physics, Witten made the conjectures about the rigidity of these elliptic operators which say that their $S^{1}$ equivariant indices on $M$ are independent of $g \in S^{1}$. See $[\mathbf{L}]$ for the early history of the subject.

These conjectures were proved by Taubes $[\mathbf{T}]$, Bott-Taubes $[\mathbf{B T}]$, and by Hirzebruch $[\mathbf{H}]$ and Krichever $[\mathbf{K}]$. Many aspects of mathematics are involved in their proofs. Taubes used analysis of Fredholm operators and Witten's interpretation of the Atiyah-Bott-Segal-Singer Lefschetz fixed point formula; Krichever used cobordism; Bott and Taubes and Hirzebruch used the Atiyah-Bott-Segal-Singer Lefschetz fixed point formula. In [Liu1], it was observed that these rigidity theorems are consequences of their modular invariance. This allowed Liu ([Liu1, 2]) to give a simple and unified proof, as well as various further generalizations, of the above conjectures of Witten. In particular, several new vanishing theorems were found in $[$ Liu1, 2].

In many situations in geometry, it is rather natural and necessary to generalize the above rigidity and vanishing theorems to the family case. For example, to use elliptic operators to study the fundamental groups of a manifold, one can reduce the question to a problem involving a family of elliptic operators. In [LM1] and [LM2], Liu and Ma proved several

\footnotetext{
${ }^{1}$ Partially supported by the Sloan Fellowship and an NSF grant.

${ }^{2}$ Partially supported by SFB 288.

${ }^{3}$ Partially supported by NSFC, MOEC and the Qiu Shi Foundation.
} 
family rigidity and vanishing theorems. Such theorems contain higher level vanishing terms which will be useful for our understanding of the relationship between group actions and fundamental groups.

To be more precise, let $M, B$ be two compact smooth manifolds, and $\pi: M \rightarrow B$ be a smooth fibration with compact fiber $X$. Assume that a compact Lie group $G$ acts fiberwise on $M$, that is, the action preserves each fiber of $\pi$. Let $P$ be a family of $G$-equivariant elliptic operators along the fiber $X$. Then the family index of $P$, in the sense of Atiyah and Singer [AS],

$$
\operatorname{Ind}(P)=\operatorname{Ker} P-\operatorname{Coker} P \in K_{G}(B),
$$

is well-defined. Note that $\operatorname{Ind}(P)$ is a family of virtual $G$-representation indexed by elements of the space $B$. Let $\widehat{G}$ denote the space of all complex irreducible representations of $G$. By [S, Proposition 2.2], we have

$$
\operatorname{Ind}(P)=\bigoplus_{V \in \widehat{G}} \operatorname{Hom}_{G}(V, \operatorname{Ind}(P)) \otimes V
$$

with $\operatorname{Hom}_{G}(V, \operatorname{Ind}(P)) \in K(B)$. We denote by $(\operatorname{Ind}(P))^{G} \in K(B)$ the $G$ invariant part of $\operatorname{Ind}(P)$.

A family of elliptic operator $P$ is said to be rigid on the equivariant Chern character level with respect to this $G$-action, if the equivariant Chern character $\operatorname{ch}_{g}(\operatorname{Ind}(P)) \in H^{*}(B)$ is independent of $g \in G$. If $\operatorname{ch}_{g}(\operatorname{Ind}(P))$ is identically zero for any $g \in G$, then we say $P$ has vanishing property on the equivariant Chern character level. More generally, we say that $P$ is rigid on the equivariant $K$-theory level, if $\operatorname{Ind}(P)=(\operatorname{Ind}(P))^{G}$. If this index is identically zero in $K_{G}(B)$, then we say that $P$ has vanishing property on the equivariant $K$-theory level. To study rigidity and vanishing properties of $\operatorname{Ind}(P)$, it is clear that we only need to restrict to the case where $G=S^{1}$. From now on we make the assumption that $G=S^{1}$.

Note that the $K$-theory level rigidity and vanishing properties are more subtle than those on the Chern character level. The reason is that, by taking the Chern character, some torsion elements involved in the index bundle might be killed. Such torsion elements may appear in the study of fundamental groups, which we hope to pursue in the future.

In [LM1], Liu and Ma proved that the elliptic genera are actually rigid on the equivariant Chern character level. Several vanishing theorems for equivariant Chern characters of these index bundles are also proved in [LM1]. Motivated by the family rigidity theorem of [LM1, Theorem 2.1], it is rather natural to expect that the elliptic genera have rigidity and vanishing properties on the equivariant $K$-theory level. The purpose of the present paper is to show that this is indeed the case. 
To achieve our purpose, we first establish a $K$-theory version of the equivariant family index theorem [LM1, Theorem 1.1] for $S^{1}$-action. However, we are not able to derive this formula directly by applying the localization formula in the equivariant $K$-theory as in [ASe], as the localizing process vill also kill the torsion elements in $K_{S^{1}}(B)$. Here instead, we combine the analysis of Wu-Zhang [WuZ, §3], which in turn relies on the technique of Bismut-Lebeau [BL], with a deformation trick of Zhang [Z, §2], which allows us to avoid the small eigenvalues problem, to establish such a formula.

To prove the main results of this paper, which are stated in Section 2.1, we will introduce some shift operators on certain vector bundles over the fixed point set of the circle action, and compare the index bundles after the shift operation. Then we get a recursive relation of these index bundles which will in turn lead us to the final result. This part is essentially a reformulation of the basic ideas of Taubes $[\mathbf{T}]$. Our main observation here is that we can directly do the shift operations on the fixed point set by applying the $K$-theory version of the equivariant family index theorem. In this way, we avoid the construction of the Dirac operator on the normal bundle in the loop space, as well as the associated analysis on the Fredholm properties of these operators (cf. [T]). Consequently, some of the shifting operations we will construct are not the same as that in [T]. This simplifies the computation significantly. In fact, in some sense the proof we will present may be considered as a rather subtle $K$-theory version of the proof of BottTaubes [BT].

Some of the results of this paper have been announced in [LMZ1]. In a subsequent paper [LMZ2], we will use the method in this paper to prove several rigidity and vanishing theorems on the equivariant $K$-theory level for Spin ${ }^{c}$-manifolds, and also for almost complex manifolds.

This paper is organized as follows. In Section 1 , we prove a $K$-theory version of the equivariant family index theorem for circle action. In Section 2 , we prove the rigidity and vanishing theorems of elliptic genera on the equivariant $K$-theory level. The proofs of the main results in Section 2 are based on two intermediate results which will be proved in Sections 3 and 4 respectively.

Acknowledgements. Part of this work was done while the authors were visiting the Morningside Center of Mathematics in Beijing during the summer of 1999. The authors would like to thank the Morningside Center for its hospitality. The second author would also like to thank the Nankai Institute of Mathematics for its hospitality. We thank the referee for careful reading and helpful comments. 


\section{A $K$-theory version of the equivariant family index theorem.}

In this section, we will prove a $K$-theory version of the equivariant family index theorem [LM1, Theorem 1.1] for $S^{1}$-actions, which will play a crucial role in the following sections. As an immediate consequence, we obtain a $K$ theory version of the famous $\widehat{A}$-vanishing theorem of Atiyah and Hirzebruch [AH] for compact connected spin manifolds with non-trivial $S^{1}$-actions. As was pointed out in the introduction, unlike the case of the usual index, we can not get the $K$-theory index formula directly by applying the localization formula in equivariant $K$-theory as in [ASe], as the localizing process will kill the torsion elements in $K_{S^{1}}(B)$. So the formula we will derive is more precise and subtle for the $S^{1}$-action case.

This section is organized as follows: In Section 1.1, we state a $K$-theory version of the equivariant family index theorem for an $S^{1}$-action on a family of spin manifolds. In Section 1.2, we prove Theorem 1.1 by applying the techniques of [BL, Sections 8, 9], [WuZ, Section 3] and a deformation trick in $[\mathbf{Z}]$. In Section 1.3, we generalize Theorem 1.1 to a somewhat more general situation. In particular, we obtain a $K$-theory version of the equivariant family index theorem for an $S^{1}$-action on a family of $\operatorname{Spin}^{c}$ Dirac operators.

\subsection{A $K$-theory version of the equivariant family index theorem.}

Let $M, B$ be two compact manifolds, and $\pi: M \rightarrow B$ a smooth fibration with compact fiber $X$ such that $\operatorname{dim} X=2 l$. Let $T X$ denote the relative tangent bundle. Let $W$ be a complex vector bundle over $M$ and $h^{W}$ an Hermitian metric on $W$.

Let $h^{T X}$ be a Riemannian metric on $T X$ and $\nabla^{T X}$ be the corresponding Levi-Civita connection on $T X$ along the fiber $X$. Then the Clifford bundle $C(T X)$ is the bundle of Clifford algebras over $M$ whose fiber at $x \in M$ is the Clifford algebra $C\left(T_{x} X\right)$ of $\left(T X, h^{T X}\right)$.

We assume that the bundle $T X$ is spin over $M$. Let $S(T X)=S^{+}(T X) \oplus$ $S^{-}(T X)$ be the spinor bundle of $T X$. We denote by $c(\cdot)$ the Clifford action of $C(T X)$ on $S(T X)$. Let $\left\{e_{i}\right\}$ be an oriented orthonormal basis of $\left(T X, h^{T X}\right)$, let $\left\{e^{i}\right\}$ be its dual basis. Let

$$
\tau=i^{l} c\left(e_{1}\right) \cdots c\left(e_{2 l}\right)
$$

be the involution of $S(T X)$. Then $\left.\tau\right|_{S^{ \pm}(T X)}= \pm 1$.

Let $\nabla^{S(T X)}$ be the Hermitian connection on $S(T X)$ induced by $\nabla^{T X}$. Let $\nabla^{W}$ be an Hermitian connection on $\left(W, h^{W}\right)$. Let $\nabla^{S(T X) \otimes W}$ be the 
connection on $S(T X) \otimes W$ along the fiber $X$ :

$$
\nabla^{S(T X) \otimes W}=\nabla^{S(T X)} \otimes 1+1 \otimes \nabla^{W} .
$$

For $b \in B$, we denote by $E_{b}, E_{ \pm, b}$ the set of $\mathcal{C}^{\infty}$-sections of $S(T X) \otimes W$, $S^{ \pm}(T X) \otimes W$ over the fiber $X_{b}$. We regard $E_{b}$ as the fiber of a smooth $\mathbf{Z}_{2}$-graded infinite dimensional vector bundle over $B$. Smooth sections of $E$ over $B$ will be identified to smooth sections of $S(T X) \otimes W$ over $M$.

Definition 1.1. Define the twisted Dirac operator to be

$$
D^{X}=\sum_{i} c\left(e_{i}\right) \nabla_{e_{i}}^{S(T X) \otimes W} .
$$

Then $D^{X}$ is a family Dirac operator which acts fiberwise on the fibers of $\pi$. For $b \in B, D_{b}^{X}$ denote the restriction of $D^{X}$ to the fiber $E_{b} . D^{X}$ interchanges $E_{+}$and $E_{-}$. Let $D_{ \pm}^{X}$ be the restrictions of $D^{X}$ to $E_{ \pm}$.

Now we assume that $S^{1}$ acts fiberwise on $M$. We will consider that $S^{1}$ acts as identity on $B$. Without loss of generality we can assume that $S^{1}$ acts on $\left(T X, h^{T X}\right)$ isometrically. We also assume that the action of $S^{1}$ lifts to $S(T X)$ and $W$, and commutes with $\nabla^{W}$. Recall that the $S^{1}$ action on $E$ is defined by $(g \cdot s)(x)=g\left(s\left(g^{-1} x\right)\right)$ for $g \in S^{1}, s \in E, x \in M$.

From [LM1, Proposition 1.1], we know that the virtual bundle over $B$,

$$
\operatorname{Ind}\left(D^{X}\right)=\operatorname{Ker} D_{+, b}^{X}-\operatorname{Ker} D_{-, b}^{X},
$$

is well-defined in the equivariant $K$-group $K_{S^{1}}(B)$.

We denote by $[n](n \in \mathbf{Z})$ the one dimensional complex vector space on which $S^{1}$ acts as multiplication by $g^{n}$ for a generator $g \in S^{1}$. By [ $\mathbf{S}$, Proposition 2.2], we know that there exist $A \in \mathbf{N}, R_{n} \in K(B)(|n| \leq A)$ such that

$$
\operatorname{Ind}\left(D^{X}\right)=\bigoplus_{|n| \leq A} R_{n} \otimes[n]
$$

as an identification of virtual $S^{1}$-bundles.

For $n \in \mathbf{Z}$, let $E_{+, b}^{n}, E_{-, b}^{n}$ be the subspaces of $E_{+, b}, E_{-, b}$ where $S^{1}$ acts as multiplication by $g^{n}$ for $g \in S^{1}$. Then we can consider $E_{b}^{n}=E_{+, b}^{n} \oplus E_{-, b}^{n}$ as the fibers of a smooth $\mathbf{Z}_{2}$-graded infinite dimensional vector bundle over $B$. Smooth sections of $E^{n}$ over $B$ will be identified with smooth sections of $S(T X) \otimes W$ over $M$ on which $S^{1}$ acts as multiplication by $g^{n}$ for $g \in S^{1}$. Then the virtual bundle over $B$,

$$
\operatorname{Ind}\left(D^{X}, n\right)=\left.\operatorname{Ker} D_{+, b}^{X}\right|_{E_{+, b}^{n}}-\left.\operatorname{Ker} D_{-, b}^{X}\right|_{E_{-, b}^{n}},
$$


is well-defined in the $K$-group $K(B)$.

By the construction of the index bundle $\operatorname{Ind}\left(D^{X}\right)$ (cf. [LM1, Proposition $1.2])$, it is well known that

$$
\begin{aligned}
\operatorname{Ind}\left(D^{X}, n\right) & =R_{n}, \quad \text { for }|n| \leq A, \\
& =0, \text { for }|n|>A .
\end{aligned}
$$

Let $F=\left\{F_{\alpha}\right\}$ be the fixed point set of the circle action on $M$. Then $\pi: F_{\alpha} \rightarrow B$ (resp. $\pi: F \rightarrow B$ ) is a smooth fibration with fiber $Y_{\alpha}$ (resp. $Y$ ), and $Y$ is a totally geodesic compact submanifold in each fiber $X$. Let $\tilde{\pi}: N \rightarrow F$ denote the normal bundle to $F$ in $M$. Then $N=T X / T Y$. We identify $N$ as the orthogonal complement of $T Y$ in $\left.T X\right|_{F}$. Let $h^{T Y}, h^{N}$ be the corresponding metrics on $T Y, N$ induced by $h^{T X}$. Then, we have the following $S^{1}$-equivariant decomposition of $T X$ when restricted to $F$ :

$$
\left.T X\right|_{F}=N_{m_{1}} \oplus \cdots \oplus N_{m_{l}} \oplus T Y,
$$

where each $N_{\gamma}$ is a complex vector bundle such that $g \in S^{1}$ acts on it by $g^{\gamma}$. To simplify the notation, we will simply write that

$$
\left.T X\right|_{F}=\oplus_{v \neq 0} N_{v} \oplus T Y,
$$

where $N_{v}$ is a complex vector bundle such that $g \in S^{1}$ acts on it by $g^{v}$ with $v \in \mathbf{Z}^{*}$. Clearly, $N=\oplus_{v \neq 0} N_{v}$. We will regard $N$ as a complex vector bundle, and write $N_{\mathbb{R}}$ for the underlying real vector bundle of $N$.

Since $N_{\mathrm{R}}$ is naturally oriented by the complex structure on $N, T Y$ is naturally oriented by the orientations of $T X$ and $N$. Similarly, let

$$
\left.W\right|_{F}=\oplus_{v} W_{v}
$$

be the $S^{1}$-equivariant decomposition of the restriction of $W$ over $F$. Here $W_{v}(v \in \mathbf{Z})$ is a complex vector bundle over $F$ on which $g \in S^{1}$ acts by $g^{v}$.

Let $C\left(N_{\mathbf{R}}\right)$ be the Clifford algebra bundle of $\left(N_{\mathbf{R}}, h^{N}\right)$. Then $\Lambda\left(\bar{N}^{*}\right)$ is a $C\left(N_{\mathbf{R}}\right)$-Clifford module. Namely, if $U \in N$, let $U^{\prime} \in \bar{N}^{*}$ correspond to $U$ by the metric $h^{T X}$. If $U \in N$, we write

$$
c(U)=\sqrt{2} U^{\prime} \wedge, \quad c(\bar{U})=-\sqrt{2} i_{\bar{U}} .
$$

On $F$, let $w_{2}(T Y), w_{2}(N)$ be the second Stiefel-Whitney classes of $T Y, N$ respectively. Let $\operatorname{det} N_{v}, \operatorname{det} N=\bigotimes_{v} \operatorname{det} N_{v}$ be the determinant line bundles of $N_{v}, N$ over $F$. Let $c_{1}(\operatorname{det} N)$ be the first Chern class of $\operatorname{det} N$. As $T X$ is spin, one gets the following identity in $H^{2}\left(F, \mathbf{Z}_{2}\right)$ :

$$
w_{2}(T Y)=w_{2}(N)=c_{1}(\operatorname{det} N) \bmod (2) .
$$


As explained in [LaM, Appendix D, p. 397], we can construct a $\operatorname{Spin}^{c}$ -principal bundle and a complex spinor bundle $S\left(T Y,(\operatorname{det} N)^{-1}\right)$ over $F$ which locally may be thought of as

$$
\begin{aligned}
& S\left(T Y,(\operatorname{det} N)^{-1}\right)=S_{0}(T Y) \otimes(\operatorname{det} N)^{-1 / 2}, \\
& \Lambda\left(\bar{N}^{*}\right)=S_{0}\left(N_{\mathbf{R}}\right) \otimes(\operatorname{det} N)^{1 / 2},
\end{aligned}
$$

where $S_{0}(T Y), S_{0}\left(N_{\mathbf{R}}\right)$ are the fundamental spinor bundles for the (possibly nonexistent) spin structure on $T Y, N_{\mathbf{R}}$, and where $(\operatorname{det} N)^{-1 / 2}$ is the (possibly nonexistent) square root of $(\operatorname{det} N)^{-1}$.

Since $Y$ is totally geodesic in $X$, the connection $\left.\nabla^{T X}\right|_{F}$ also preserves the decomposition (1.8) of $T X$ over $F$. Let $\nabla^{T Y}, \nabla^{N}, \nabla^{N_{v}}$ be the corresponding induced connections on $T Y, N$ and $N_{v}$. Let $\nabla^{\Lambda\left(\bar{N}^{*}\right)}$ be the connection on $\Lambda\left(\bar{N}^{*}\right)$ induced by $\nabla^{N}$.

Let $\nabla^{(\operatorname{det} N)^{-1}}$ be the connection on $(\operatorname{det} N)^{-1}$ induced by $\nabla^{N}$. The connections $\nabla^{(\operatorname{det} N)^{-1}}$ and $\nabla^{T Y}$ induce an Hermitian connection $\nabla^{S\left(T Y,(\operatorname{det} N)^{-1}\right)}$ on $S\left(T Y,(\operatorname{det} N)^{-1}\right)$. In fact, locally, both $S_{0}(T Y)$ and $(\operatorname{det} N)^{-1 / 2}$ exist and carry a canonical connection induced by $\nabla^{T Y}, \nabla^{(\operatorname{det} N)^{-1}}$. We give the bundle $S\left(T Y,(\operatorname{det} N)^{-1}\right)$ the tensor product connection. It is standard that this connection is well defined globally (cf. [LaM, Appendix D]).

Let $\left(e_{1}, \ldots, e_{2 l^{\prime}}\right),\left(e_{2 l^{\prime}+1}, \ldots, e_{2 l}\right)$ be the corresponding oriented orthonormal bases of $T Y$ and $N_{\mathbf{R}}$. Then $\left(e_{1}, \ldots, e_{2 l}\right)$ is an oriented orthonormal basis of $T X$. The $\mathbf{Z}_{2}$-gradings on $S\left(T Y,(\operatorname{det} N)^{-1}\right), \Lambda\left(\bar{N}^{*}\right)$ are defined by the involutions $i^{l^{\prime}} c\left(e_{1}\right) \ldots c\left(e_{2 l^{\prime}}\right)$, and $\tau^{N}=i^{l-l^{\prime}} c\left(e_{2 l^{\prime}+1}\right) \ldots c\left(e_{2 l}\right)$ respectively. Also note that under the involution $\tau^{N}$,

$$
\Lambda^{\text {even }}\left(\bar{N}^{*}\right)=\left(\Lambda\left(\bar{N}^{*}\right)\right)^{+}, \quad \Lambda^{\text {odd }}\left(\bar{N}^{*}\right)=\left(\Lambda\left(\bar{N}^{*}\right)\right)^{-} .
$$

From the above discussion, we see that there is a natural isomorphism between $\mathbf{Z}_{2}$-graded $C(T X)$-Clifford modules over $F$,

$$
\left.S\left(T Y,(\operatorname{det} N)^{-1}\right) \widehat{\otimes} \Lambda\left(\bar{N}^{*}\right) \simeq S(T X)\right|_{F} .
$$

Here we denote the $\mathbf{Z}_{2}$-graded tensor product by $\widehat{\otimes}$ (cf. [LaM, p. 11]). Furthermore, since $\nabla^{N}$ is $S^{1}$-invariant, one deduces easily that

$$
\left.\nabla^{S(T X)}\right|_{F}=\nabla^{S\left(T Y,(\operatorname{det} N)^{-1}\right)} \widehat{\otimes} 1+1 \widehat{\otimes} \nabla^{\Lambda\left(\bar{N}^{*}\right)} .
$$

Let $V$ be an Hermitian vector bundle over $F$. Let $\nabla^{V}$ be an Hermitian connection on $V$. From now on, we will also denote by $D^{Y} \otimes V$ the family twisted Dirac operator on $S\left(T Y,(\operatorname{det} N)^{-1}\right) \otimes V$ on $F$, and $D^{Y_{\alpha}} \otimes V$ 
its restriction to $F_{\alpha}$. Namely, let $\nabla^{S\left(T Y,(\operatorname{det} N)^{-1}\right) \otimes V}$ be the tensor product connection on $S\left(T Y,(\operatorname{det} N)^{-1}\right) \otimes V$ induced by $\nabla^{S\left(T Y,(\operatorname{det} N)^{-1}\right)}$ and $\nabla^{V}$. Then

$$
D^{Y} \otimes V=\sum_{i=1}^{2 l^{\prime}} c\left(e_{i}\right) \nabla_{e_{i}}^{S\left(T Y,(\operatorname{det} N)^{-1}\right) \otimes V} .
$$

We use the notation

$$
\begin{aligned}
& \operatorname{Sym}_{q}(V)=\sum_{n=0}^{+\infty} q^{n} \operatorname{Sym}^{n}(V) \in K(F)[[q]], \\
& \Lambda_{q}(V)=\sum_{n=0}^{+\infty} q^{n} \Lambda^{n}(V) \in K(F)[[q]]
\end{aligned}
$$

for the symmetric and exterior power operations in $K(F)[[q]]$ respectively.

Introduce the notations:

$$
\begin{gathered}
R(q)=q^{\frac{1}{2} \sum_{v}|v| \operatorname{dim} N_{v}} \bigotimes_{v>0}\left(\operatorname{Sym}_{q^{v}}\left(N_{v}\right) \otimes \operatorname{det} N_{v}\right) \\
\otimes \bigotimes_{v<0} \operatorname{Sym}_{q^{-v}}\left(\bar{N}_{v}\right) \otimes\left(\sum_{v} q^{v} W_{v}\right)=\sum_{n} R_{n} q^{n}, \\
R^{\prime}(q)=q^{-\frac{1}{2} \sum_{v}|v| \operatorname{dim} N_{v}} \bigotimes_{v>0} \operatorname{Sym}_{q^{-v}}\left(\bar{N}_{v}\right) \\
\otimes \bigotimes_{v<0}\left(\operatorname{Sym}_{q^{v}}\left(N_{v}\right) \otimes \operatorname{det} N_{v}\right) \otimes\left(\sum_{v} q^{v} W_{v}\right)=\sum_{n} R_{n}^{\prime} q^{n} .
\end{gathered}
$$

Note that by $[\mathbf{A H}]$ (also cf. the argument after (2.27)), one knows that, as $T X$ is spin,

$$
\sum_{v} v \operatorname{dim} N_{v}=0 \bmod (2)
$$

We can now state the main result of this section as follows.

Theorem 1.1. For $n \in \mathbf{Z}$, we have the following identity in $K(B)$ :

$$
\begin{aligned}
\operatorname{Ind}\left(D^{X}, n\right) & =\sum_{\alpha}(-1)^{\sum_{0<v} \operatorname{dim} N_{v}} \operatorname{Ind}\left(D^{Y_{\alpha}} \otimes R_{n}\right) \\
& =\sum_{\alpha}(-1)^{\sum_{v<0} \operatorname{dim} N_{v}} \operatorname{Ind}\left(D^{Y_{\alpha}} \otimes R_{n}^{\prime}\right) .
\end{aligned}
$$


If we take $W=\mathbf{C}$ the trivial line bundle over $M$, then the operator $D^{X} \otimes \mathbf{C}$ is exactly the canonical Dirac operator $D^{X}$. The following consequence generalizes the famous $\widehat{A}$-vanishing theorem of Atiyah and Hirzebruch $[\mathbf{A H}]$ for compact connected spin manifolds with non-trivial $S^{1}$ actions to the family case.

Corollary 1.1. If $M$ is connected, and the $S^{1}$ action is nontrivial, then for the family of the canonical Dirac operators $D^{X}$ along the fiber $X$, one has

$$
\text { Ind }\left(D^{X}\right)=0 \text { in } K_{S^{1}}(B) .
$$

Proof: If the $S^{1}$ action is locally free, then by Theorem 1.1, we get directly (1.18). Otherwise, since on each $F_{\alpha}$,

$$
\sum_{v}|v| \operatorname{dim} N_{v}>0
$$

one deduces easily from Theorem 1.1 that

$$
\text { Ind }\left(D^{X}, n\right)=0 \quad \text { in } \quad K(B)
$$

for any $n \in \mathbf{Z}$, from which (1.18) follows.

\subsection{Proof of Theorem 1.1.}

In this subsection, we prove Theorem 1.1. The proof, which is contained here for completeness of the present paper, is modeled on [ $\mathbf{W u Z}$, Section 3] which in turn relies on the paper of Bismut and Lebeau [BL].

This subsection is organized as follows. In Section 1.2.1, we recall a result from [WuZ, Proposition 3.2] concerning the Witten deformation on flat space. In Section 1.2.2, we establish a Taylor expansion of $D^{X}+\sqrt{-1} T c(H)$ near the fixed point set $F$, where $H$ is the Killing vector field on $M$ generated by the circle action. In Section 1.2.3, by using the techniques of [WuZ, Section 3] and [BL, Section 9], we establish various estimates for certain operators induced from $D^{X}+\sqrt{-1} T c(H)$. In Section 1.2.4, we prove Theorem 1.1 by using a deformation trick in $[\mathbf{Z}]$.

1.2.1. Witten's deformation on flat spaces. Let $H$ be the canonical basis of $\operatorname{Lie}\left(S^{1}\right)=\mathbf{R}$, i.e., for $t \in \mathbf{R}, \exp (t H)=e^{2 \pi i t} \in S^{1}$. Let $W$ be a complex vector space of dimension $n$ with an Hermitian form. Let $\rho$ be a unitary representation of the circle group $S^{1}$ on $W$ such that all the weights are nonzero. Suppose $W^{ \pm}$are the subspaces of $W$ corresponding to positive 
and negative weights respectively, with $\operatorname{dim}_{\mathbf{C}} W^{-}=\nu, \operatorname{dim}_{\mathbf{C}} W^{+}=n-\nu$. Let $z=\left\{z^{i}\right\}$ be the complex linear coordinates on $W$ such that the Hermitian structure on $W$ takes the standard form and $\rho$ is diagonal with weights $\lambda_{i} \in \mathbf{Z} \backslash\{0\}(1 \leq i \leq n)$, and $\lambda_{i}<0$ for $i \leq \nu$. The Lie algebra action on $W$ is given by the vector field

$$
H=2 \pi \sqrt{-1} \sum_{i=1}^{n} \lambda_{i}\left(z^{i} \frac{\partial}{\partial z^{i}}-\bar{z}^{i} \frac{\partial}{\partial \bar{z}^{i}}\right) .
$$

Set

$$
K^{ \pm}(W)=\operatorname{Sym}\left(\left(W^{ \pm}\right)^{*}\right) \otimes \operatorname{Sym}\left(W^{\mp}\right) \otimes \operatorname{det}\left(W^{\mp}\right) .
$$

Let $E$ be a finite dimensional complex vector space with an Hermitian form and suppose $E$ carries a unitary representation of $S^{1}$.

Let $\bar{\partial}$ be the twisted Dolbeault operator acting on $\Omega^{0, *}(W, E)=$ $\Gamma\left(\Lambda\left(\bar{W}^{*}\right) \otimes E\right)$, the set of smooth sections of $\Lambda\left(\bar{W}^{*}\right) \otimes E$ on $W$. And let $\bar{\partial}^{*}$ be its formal adjoint. Let $D=\sqrt{2}\left(\bar{\partial}+\bar{\partial}^{*}\right)$. Let $c(H)$ be the Clifford action of $H$ on $\Lambda\left(\bar{W}^{*}\right)$ defined as in (1.10). Let $L_{H}$ be the Lie derivative along $H$ acting on $\Omega^{0, *}(W, E)$.

The following result was proved in [WuZ, Proposition 3.2].

Proposition 1.1. 1. A basis of the space of $L^{2}$-solutions of $D+\sqrt{-1} c(H)$ (resp. $D-\sqrt{-1} c(H))$ on the space of $\mathcal{C}^{\infty}$ sections of $\Lambda\left(\bar{W}^{*}\right)$ is given by

$$
\left(\prod_{i=1}^{\nu} z_{i}^{k_{i}}\right)\left(\prod_{i=\nu+1}^{n} \bar{z}_{i}^{k_{i}}\right) e^{-\sum_{i=1}^{n} \pi\left|\lambda_{i}\right|\left|z_{i}\right|^{2}} d \bar{z}_{\nu+1} \cdots d \bar{z}_{n} \quad\left(k_{i} \in \mathbf{N}\right)
$$

with weight $\sum_{i=1}^{\nu} k_{i}\left|\lambda_{i}\right|+\sum_{i=\nu+1}^{n}\left(k_{i}+1\right)\left|\lambda_{i}\right|$ (resp.

$$
\left(\prod_{i=1}^{\nu} \bar{z}_{i}^{k_{i}}\right)\left(\prod_{i=\nu+1}^{n} z_{i}^{k_{i}}\right) e^{-\sum_{i=1}^{n} \pi\left|\lambda_{i}\right|\left|z_{i}\right|^{2}} d \bar{z}_{1} \cdots d \bar{z}_{\nu} \quad\left(k_{i} \in \mathbf{N}\right)
$$

with weight $\left.-\sum_{i=\nu+1}^{n} k_{i}\left|\lambda_{i}\right|-\sum_{i=1}^{\nu}\left(k_{i}+1\right)\left|\lambda_{i}\right|\right)$.

So the space of $L^{2}$-solution of a given weight of $D+\sqrt{-1} c(H)$ (resp. $D-\sqrt{-1} c(H))$ on the space of $\mathcal{C}^{\infty}$ sections of $\Lambda\left(\bar{W}^{*}\right) \otimes E$ is finite dimensional. The direct sum of these weight spaces is isomorphic to $K^{-}(W) \otimes E$ (resp. $\left.K^{+}(W) \otimes E\right)$ as representations of $S^{1}$.

2. When restricted to an eigenspace of $L_{H}$, the operator $D+\sqrt{-1} c(H)$ (resp. $D-\sqrt{-1} c(H)$ ) has discrete eigenvalues. 


\subsubsection{A Taylor expansion of certain deformed operators near the} fixed-point set. In this subsection, we will use the notation of Section 1.1. We assume temporarily that $B$ is a point, and $Y$ is the fixed point set of the $S^{1}$ action on $X$.

Following [BL, Section 8e)] and [WuZ, Section 3.2], we now describe a coordinate system on $X$ near $Y$. For $\varepsilon>0$, set $B_{\varepsilon}=\{Z \in N ;|Z|<\varepsilon\}$. Since $X$ and $Y$ are compact, there exists $\varepsilon_{0}>0$ such that for $0<\varepsilon \leq \varepsilon_{0}$, the exponential map $(y, Z) \in N \rightarrow \exp _{y}^{X}(Z) \in X$ is a diffeomorphism from $B_{\varepsilon}$ onto a tubular neighborhood $U_{\varepsilon}$ of $Y$ in $X$. From now on, we identify $B_{\varepsilon}$ with $U_{\varepsilon}$ and use the notation $x=(y, Z)$ instead of $x=\exp _{y}^{X}(Z)$. Finally, we identify $y \in Y$ with $(y, 0) \in N$. Let $\widetilde{\pi}^{*}\left(\left.(S(T X) \otimes W)\right|_{Y}\right)$ be the vector bundle on $N$ obtained by pulling back of $\left.(S(T X) \otimes W)\right|_{Y}$ for $\widetilde{\pi}: N \rightarrow Y$.

Let $h^{T Y}, h^{N}$ be the corresponding metrics on $T Y$ and $N$ induced by $h^{T X}$. Let $d v_{X}, d v_{Y}$ and $d v_{N}$ be the corresponding volume elements on $\left(T X, h^{T X}\right),\left(T Y, h^{T Y}\right)$ and $\left(N, h^{N}\right)$. Let $k(y, Z)\left((y, Z) \in B_{\varepsilon}\right)$ be the smooth positive function defined by

$$
d v_{X}(y, Z)=k(y, Z) d v_{Y}(y) d v_{N_{y}}(Z) .
$$

Then $k(y)=1$ and $\frac{\partial k}{\partial Z}(y)=0$ for $y \in Y$; the latter follows from [BL, Proposition 8.9] and the fact that $Y$ is totally geodesic in $X$.

For $x=(y, Z) \in U_{\varepsilon_{0}}$, we will identify $S(T X)_{x}$ with $S(T X)_{y}$ and $W_{x}$ with $W_{y}$ by the parallel transport with respect to the $S^{1}$-invariant connections $\nabla^{S(T X)}$ and $\nabla^{W}$ respectively, along the geodesic $t \rightarrow(y, t Z)$. The induced identification of $(S(T X) \otimes W)_{x}$ with $(S(T X) \otimes W)_{y}$ preserves the metric and the $\mathbf{Z}_{2}$-grading, and is moreover $S^{1}$-equivariant. Consequently, $D^{X}$ can be considered as an operator acting on the sections of the bundle $\widetilde{\pi}^{*}((S(T X) \otimes$ $W)\left.\right|_{Y}$ ) over $B_{\varepsilon_{0}}$ commuting with the $S^{1}$ action.

For $\varepsilon>0$, let $\mathbf{H}\left(B_{\varepsilon}\right)$ (resp. $\mathbf{H}(N)$ ) be the set of smooth sections of $\tilde{\pi}^{*}\left(\left.(S(T X) \otimes W)\right|_{Y}\right)$ on $B_{\varepsilon}$ (resp. on the total bundle of $\left.N\right)$. If $f, g \in \mathbf{H}(N)$ have compact supports, we will write

$$
\langle f, g\rangle=\left(\frac{1}{2 \pi}\right)^{\operatorname{dim} X} \int_{Y}\left(\int_{N}\langle f, g\rangle(y, Z) d v_{N_{y}}(Z)\right) d v_{Y}(y) .
$$

Then $k^{1 / 2} D^{X} k^{-1 / 2}$ is a (formally) self-adjoint operator on $\mathbf{H}(N)$.

The connection $\nabla^{N}$ on $N$ induces a splitting $T N=N \oplus T^{H} N$, where $T^{H} N$ is the horizontal part of $T N$ with respect to $\nabla^{N}$. Moreover, since $Y$ is totally geodesic, this splitting, when restricted to $Y$, is preserved by the connection $\nabla^{T X}$ on $\left.T X\right|_{Y}$. Let $\widetilde{\nabla}$ be the connection on $\left.(S(T X) \otimes W)\right|_{Y}$ 
induced by the restriction of $\nabla^{S(T X) \otimes W}$ to $Y$. We will still denote by $\tilde{\nabla}$ the lift of the connection $\widetilde{\nabla}$ to $\widetilde{\pi}^{*}\left(\left.(S(T X) \otimes W)\right|_{Y}\right)$.

We choose a local orthonormal basis of $T X$ such that $e_{1}, \ldots, e_{2 l^{\prime}}$ form a basis of $T Y$, and $e_{2 l^{\prime}+1}, \ldots, e_{2 l}$, that of $N_{\mathbf{R}}$. Denote the horizontal lift of $e_{i}$ $\left(1 \leq i \leq 2 l^{\prime}\right)$ to $T^{H} N$ by $e_{i}^{H}$. As in [BL, Definition 8.16] and [ WuZ, (3.15)], we define

$$
D^{H}=\sum_{i=1}^{2 l^{\prime}} c\left(e_{i}\right) \widetilde{\nabla}_{e_{i}^{H}}, \quad D^{N}=\sum_{i=2 l^{\prime}+1}^{2 l} c\left(e_{i}\right) \widetilde{\nabla}_{e_{i}} .
$$

Clearly, $D^{N}$ acts along the fibers of $N$. Let $\bar{\partial}^{N}$ be the $\bar{\partial}$-operator along the fibers of $N$, and let $\bar{\partial}^{N *}$ be its formal adjoint with respect to (1.23). By (1.13), it is easy to see that $D^{N}=\sqrt{2}\left(\bar{\partial}^{N}+\bar{\partial}^{N *}\right)$. Both $D^{N}$ and $D^{H}$ are self-adjoint with respect to (1.23).

For $T>0$, we define a scaling $f \in \mathbf{H}\left(B_{\varepsilon_{0}}\right) \rightarrow S_{T} f \in \mathbf{H}\left(B_{\varepsilon_{0} \sqrt{T}}\right)$ by

$$
S_{T} f(y, Z)=f\left(y, \frac{Z}{\sqrt{T}}\right), \quad(y, Z) \in B_{\varepsilon_{0} \sqrt{T}} .
$$

For a first order differential operator

$$
Q_{T}=\sum_{i=1}^{2 l^{\prime}} a_{T}^{i}(y, Z) \widetilde{\nabla}_{e_{i}^{H}}+\sum_{i=2 l^{\prime}+1}^{2 l} b_{T}^{i}(y, Z) \widetilde{\nabla}_{e_{i}}+c_{T}(y, Z)
$$

acting on $\mathbf{H}\left(B_{\varepsilon_{0} \sqrt{T}}\right)$, where $a_{T}^{i}, \quad b_{T}^{i}$ and $c_{T}$ are endomorphisms of $\tilde{\pi}^{*}\left(\left.(S(T X) \otimes W)\right|_{Y}\right)$, we write

$$
Q_{T}=O\left(|Z|^{2} \partial^{N}+|Z| \partial^{H}+|Z|+|Z|^{p}\right), \quad(p \in \mathbf{N}),
$$

if there is a constant $C>0$ such that for any $T \geq 1,(y, Z) \in B_{\varepsilon_{0} \sqrt{T}}$, we have

$$
\begin{aligned}
& \left|a_{T}^{i}(y, Z)\right| \leq C|Z| \quad\left(1 \leq i \leq 2 l^{\prime}\right) \\
& \left|b_{T}^{i}(y, Z)\right| \leq C|Z|^{2} \quad\left(2 l^{\prime}+1 \leq i \leq 2 l\right), \\
& \left|c_{T}(y, Z)\right| \leq C\left(|Z|+|Z|^{p}\right) .
\end{aligned}
$$

Let $J_{H}$ be the representation of $\operatorname{Lie}\left(S^{1}\right)$ on $N$. Then $Z \rightarrow J_{H} Z$ is a Killing vector field on $N$. We have the following analog of [BL, Theorem 8.18] and [WuZ, Proposition 3.3]. 
Proposition 1.2. As $T \rightarrow+\infty$,

$$
\begin{aligned}
S_{T} k^{1 / 2}\left(D^{X}+\sqrt{-1}\right. & T c(H)) k^{-1 / 2} S_{T}^{-1}=\sqrt{T}\left(D^{N}+\sqrt{-1} c\left(J_{H} Z\right)\right) \\
+ & D^{H}+\frac{1}{\sqrt{T}} O\left(|Z|^{2} \partial^{N}+|Z| \partial^{H}+|Z|+|Z|^{3}\right)
\end{aligned}
$$

Proof: Since $Y$ is totally geodesic in $X$ and the actions of $S^{1}$ on $N$ and $M$ commute with the exponential map, one can proceed as in the proof of [WuZ, Proposition 3.3] and [BL, Section 8] to get (1.29).

By Proposition 1.1, the solution space of the operator $D^{N}+\sqrt{-1} c\left(J_{H} Z\right)$ along the fiber $N_{y}(y \in F)$ is (the $L^{2}$-completion of) $K^{-}\left(N_{y}\right) \otimes W_{y}$. They form an infinite dimensional Hermitian complex vector bundle $\left.K^{-}(N) \otimes W\right|_{F}$ over $Y$, with the Hermitian connection $\nabla^{Y}$ induced from those on $N$ and $\left.W\right|_{Y} \rightarrow Y$.

Let $\mathbf{H}^{0}(Y)$ be the Hilbert space of square-integrable sections of $\left.\left(S\left(T Y,(\operatorname{det} N)^{-1}\right) \otimes K^{-}(N) \otimes W\right)\right|_{Y}$, and $\mathbf{H}^{0}(N)$, that of the bundle $\widetilde{\pi}^{*}\left(\left.(S(T X) \otimes W)\right|_{Y}\right)$, equipped with the corresponding Hermitian forms. By using (1.12), we define an embedding $\psi: \mathbf{H}^{0}(Y) \rightarrow \mathbf{H}^{0}(N)$ by

$$
\psi: \alpha \otimes \beta \in \mathbf{H}^{0}(Y) \rightarrow \widetilde{\pi}^{*} \alpha \wedge \tau(\beta) \in \mathbf{H}^{0}(N) .
$$

Here $\alpha \in \Gamma\left(Y, S\left(T Y,(\operatorname{det} N)^{-1}\right)\right), \beta \in L^{2}\left(\left.K^{-}(N) \otimes W\right|_{Y}\right)$ and $\tau$ is the isometry from $L^{2}\left(\left.K^{-}(N) \otimes W\right|_{Y}\right)$ to $L^{2}\left(\widetilde{\pi}^{*}\left(\left.\left(\Lambda\left(\bar{N}^{*}\right) \otimes W\right)\right|_{Y}\right)\right)$ given by Proposition 1.1. Clearly, $\psi$ is an isometry onto its image which we denote by $\mathbf{H}^{\prime, 0}$. Let $p: \mathbf{H}^{0}(N) \rightarrow \mathbf{H}^{\prime, 0}$ be the orthogonal projection. Then we have the following analog of [BL, Theorem 8.21] and [WuZ, Proposition 3.4], which can be proved in the same way as in [BL] and [WuZ].

Proposition 1.3. The following identity for operators acting on $\mathbf{H}^{0}(Y)$ holds :

$$
\psi^{-1} p D^{H} p \psi=D^{Y} \otimes R(1)
$$

where $R(1)$ is defined in (1.15).

1.2.3. Estimates of the operators as $\boldsymbol{T} \rightarrow+\infty$. We still assume temporarily that $B$ is a point. For $p \geq 0$, let $\mathbf{H}^{p}(X), \mathbf{H}^{p}(N)$ and $\mathbf{H}^{p}(Y)$ be the $p$-th Sobolev spaces of sections of the bundles $S(T X) \otimes W \rightarrow X$, $\tilde{\pi}^{*}\left(\left.(S(T X) \otimes W)\right|_{Y}\right) \rightarrow N$ and $\left.S\left(T Y,(\operatorname{det} N)^{-1}\right) \otimes K^{-}(N) \otimes W\right|_{Y} \rightarrow Y$ respectively. The group $S^{1}$ acts on all these spaces. For any $\xi \in \mathbf{Z}$, let 
$\mathbf{H}_{\xi}^{p}(X), \mathbf{H}_{\xi}^{p}(N)$ and $\mathbf{H}_{\xi}^{p}(Y)$ be the corresponding subspaces of weight $\xi \in \mathbf{Z}$. Recall that the constant $\varepsilon_{0}>0$ is defined in last subsection. We now take $\left.\epsilon \in] 0, \frac{\varepsilon_{0}}{2}\right]$, which is small enough for each eigenvalue of $L_{H}$ we will consider, but otherwise can be assumed to be fixed. Let $\rho: \mathbf{R} \rightarrow[0,1]$ be a smooth function such that

$$
\rho(a)=\left\{\begin{array}{lll}
1 & \text { if } & a \leq 1 / 2 \\
0 & \text { if } & a \geq 3 / 4
\end{array}\right.
$$

For $Z \in N$, set $\rho_{\epsilon}(Z)=\rho\left(\frac{|Z|}{\epsilon}\right)$. Let $\alpha \in \Gamma\left(Y, S\left(T Y,(\operatorname{det} N)^{-1}\right)\right), \beta \in$ $L^{2}\left(\left.K^{-}(N) \otimes W\right|_{Y}\right), \sigma=\alpha \otimes \beta$. We define a linear map $I_{T, \xi}: \mathbf{H}_{\xi}^{p}(Y) \rightarrow \mathbf{H}_{\xi}^{p}(N)$ by

$$
\sigma \in \mathbf{H}_{\xi}^{p}(Y) \longmapsto I_{T, \xi} \sigma=\frac{\rho_{\epsilon}\|\beta\|_{0}}{\left\|\rho_{\epsilon} S_{T}^{-1}(\tau(\beta))\right\|_{0}} \widetilde{\pi}^{*} \alpha \wedge S_{T}^{-1}(\tau(\beta)) \in \mathbf{H}_{\xi}^{p}(N) .
$$

Let the image of $I_{T, \xi}$ from $\mathbf{H}_{\xi}^{p}(Y)$ be $\mathbf{H}_{T, \xi}^{p}(N)=I_{T, \xi} \mathbf{H}_{\xi}^{p}(Y) \subset \mathbf{H}_{\xi}^{p}(N)$. Denote the orthogonal complement of $\mathbf{H}_{T, \xi}^{0}(N)$ in $\mathbf{H}_{\xi}^{0}(N)$ by $\mathbf{H}_{T, \xi}^{0, \perp}(N)$, and let $\mathbf{H}_{T, \xi}^{p, \perp}(N)=\mathbf{H}_{\xi}^{p}(N) \cap \mathbf{H}_{T, \xi}^{0, \perp}(N)$. Let $p_{T, \xi}$ and $p_{T, \xi}^{\perp}$ be the orthogonal projections from $\mathbf{H}_{\xi}^{0}(N)$ onto $\mathbf{H}_{T, \xi}^{0}(N)$ and $\mathbf{H}_{T, \xi}^{0, \perp}(N)$ respectively.

Since the bundle $S(T X) \otimes W$ over $U_{\varepsilon_{0}}$ is identified with

$$
\tilde{\pi}^{*}\left(\left.\left(S\left(T Y,(\operatorname{det} N)^{-1}\right) \otimes \Lambda\left(\bar{N}^{*}\right) \otimes W\right)\right|_{Y}\right)
$$

over $B_{\varepsilon_{0}}$, we can consider $k^{-1 / 2} I_{T, \xi} \sigma$ as an element of $\mathbf{H}_{\xi}^{p}(X)$ for $\sigma \in \mathbf{H}_{\xi}^{p}(Y)$. Define the linear isometry map $J_{T, \xi}$ by

$$
\sigma \in \mathbf{H}_{\xi}^{p}(Y) \longmapsto J_{T, \xi} \sigma=k^{-1 / 2} I_{T, \xi} \sigma \in \mathbf{H}_{\xi}^{p}(X) .
$$

Let $\mathbf{H}_{T, \xi}^{p}(X)=J_{T, \xi} \mathbf{H}_{\xi}^{p}(Y)$ be the image. Denote the orthogonal complement of $\mathbf{H}_{T, \xi}^{0}(X)$ in $\mathbf{H}_{\xi}^{0}(X)$ by $\mathbf{H}_{T, \xi}^{0, \perp}(X)$, and let $\mathbf{H}_{T, \xi}^{p, \perp}(X)=\mathbf{H}_{\xi}^{p}(X) \cap \mathbf{H}_{T, \xi}^{0, \perp}(X)$. Let $\bar{p}_{T, \xi}$ and $\bar{p}_{T, \xi}^{\perp}$ be the orthogonal projections from $\mathbf{H}_{\xi}^{0}(X)$ onto $\mathbf{H}_{T, \xi}^{0}(X)$ and $\mathbf{H}_{T, \xi}^{0, \perp}(X)$ respectively. It is clear that $\bar{p}_{T, \xi}=k^{-1 / 2} p_{T, \xi} k^{1 / 2}$.

For any (possibly unbounded) operator $A$ on $\mathbf{H}_{\xi}^{0}(X)$, write

$$
A=\left(\begin{array}{ll}
A^{(1)} & A^{(2)} \\
A^{(3)} & A^{(4)}
\end{array}\right)
$$

according to the decomposition $\mathbf{H}_{\xi}^{0}(X)=\mathbf{H}_{T, \xi}^{0}(X) \oplus \mathbf{H}_{T, \xi}^{0, \perp}(X)$, i.e., $A^{(1)}=$ $\bar{p}_{T, \xi} A \bar{p}_{T, \xi}, A^{(2)}=\bar{p}_{T, \xi} A \bar{p}_{T, \xi}^{\perp}, A^{(3)}=\bar{p}_{T, \xi}^{\perp} A \bar{p}_{T, \xi}$ and $A^{(4)}=\bar{p}_{T, \xi}^{\perp} A \bar{p}_{T, \xi}^{\perp}$. 
Let $D_{T}=D^{X}+\sqrt{-1} T c(H)$, where now $H$ denotes the Killing vector field on $M$ generated by the circle action. Let $D_{T, \xi}$ and $D_{\xi}^{Y}$ be the restrictions of the operators $D_{T}$ and $D^{Y} \otimes R(1)$ on $\mathbf{H}_{\xi}^{0}(X)$ and $\mathbf{H}_{\xi}^{0}(Y)$ respectively.

Proposition 1.4. 1. As $T \rightarrow+\infty$,

$$
J_{T, \xi}^{-1} D_{T, \xi}^{(1)} J_{T, \xi}=D_{\xi}^{Y}+O\left(\frac{1}{\sqrt{T}}\right)
$$

where $O\left(\frac{1}{\sqrt{T}}\right)$ denotes a first order differential operator whose coefficients are dominated by $\frac{C}{\sqrt{T}}(C>0)$.

2. For each $\xi \in \mathbf{Z}$, there exists $C>0$ such that for any $T \geq 1, \sigma \in \mathbf{H}_{T, \xi}^{1, \perp}(X)$, $\sigma^{\prime} \in \mathbf{H}_{T, \xi}^{1}(X)$, we have

$$
\begin{aligned}
& \left\|D_{T, \xi}^{(2)} \sigma\right\|_{0} \leq C\left(\frac{\|\sigma\|_{1}}{\sqrt{T}}+\|\sigma\|_{0}\right) \\
& \left\|D_{T, \xi}^{(3)} \sigma^{\prime}\right\|_{0} \leq C\left(\frac{\left\|\sigma^{\prime}\right\|_{1}}{\sqrt{T}}+\left\|\sigma^{\prime}\right\|_{0}\right) .
\end{aligned}
$$

3. For each $\xi \in \mathbf{Z}$, there exist $\left.\epsilon \in] 0, \frac{\varepsilon_{0}}{2}\right], T_{0}>0, C>0$ such that for any $T \geq T_{0}, \sigma \in \mathbf{H}_{T, \xi}^{1, \perp}(X)$, we have

$$
\left\|D_{T, \xi}^{(4)} \sigma\right\|_{0} \geq C\left(\|\sigma\|_{1}+\sqrt{T}\|\sigma\|_{0}\right) .
$$

Proof: Proposition 1.4 is the analogue of [WuZ, Proposition 3.5] and can be proved in the same way as in [WuZ, pp. 165-166], which in turn relies on $[\mathbf{B L}$, Section 9].

1.2.4. Proof of Theorem 1.1. We now go back to the family case. The important observation is that the analysis in the above two subsections works well for the fiberwise (twisted) Dirac operators.

For any $u \in \mathbf{R}$, we write

$$
D_{T, \xi}(u)=D_{T, \xi}^{(1)}+D_{T, \xi}^{(4)}+u\left(D_{T, \xi}^{(2)}+D_{T, \xi}^{(3)}\right): E \rightarrow E .
$$

The following lemma plays a key role in our proof of Theorem 1.1.

Lemma 1.1. There exists $T_{1}>0$ such that for any $u \in[0,1]$ and $T \geq T_{1}$, $D_{T, \xi}(u)$ is a continuous family of Fredholm operators over $B$. 
Proof: From Proposition 1.4, one deduces (cf. [Z, Lemma 2.2]) that there exist $C_{1}, C_{2}>0$ such that for $u \in[0,1], s \in E$ and $T$ large enough,

$$
\left\|D_{T, \xi} s-D_{T, \xi}(u) s\right\|_{0} \leq \frac{C_{1}}{\sqrt{T}}\left\|D_{T, \xi} s\right\|_{0}+C_{2}\|s\|_{0}
$$

From (1.40) and the Fredholm property of $D_{T, \xi}$, one obtains the Fredholm property of $D_{T, \xi}(u)$ for sufficiently large $T$.

Recall that the index bundle construction $[\mathbf{A S}]$ applies well to continuous families of Fredholm operators and that the homotopy invariance property for the index bundle still holds in this situation.

From Lemma 1.1, one then gets the following identity of index bundles, when $T$ is large enough,

$$
\begin{aligned}
\operatorname{Ind}\left(D_{\xi}^{X}\right) & =\operatorname{Ind}\left(D_{T, \xi}\right)=\operatorname{Ind}\left(D_{T, \xi}(0)\right) \\
& =\operatorname{Ind}\left(D_{T, \xi}^{(1)}\right)+\operatorname{Ind}\left(D_{T, \xi}^{(4)}\right) \text { in } K(B),
\end{aligned}
$$

where in the last line, $\operatorname{Ind}\left(D_{T, \xi}^{(1)}\right)\left(\operatorname{resp} . \operatorname{Ind}\left(D_{T, \xi}^{(4)}\right)\right)$ is now regarded as a family of Fredholm operators mapping from $\mathbf{H}_{T, \xi}^{0}(X)$ (resp. $\left.\mathbf{H}_{T, \xi}^{0, \perp}(X)\right)$ to $\mathbf{H}_{T, \xi}^{0}(X)\left(\operatorname{resp} . \mathbf{H}_{T, \xi}^{0, \perp}(X)\right)$.

On the other hand, by the third part of Proposition 1.4, when $T$ is large enough, one has obviously that

$$
\operatorname{Ind}\left(D_{T, \xi}^{(4)}\right)=0 \text { in } K(B),
$$

Let $D_{T, \xi, \alpha}^{(1)}$ be the restriction of $D_{T, \xi}^{(1)}$ on $F_{\alpha}$. From (1.30), (1.36) and the definition of $J_{T, \xi}$, one deduces easily that when $T$ is large enough, one has,

$$
\begin{aligned}
& \operatorname{Ind}\left(D_{T, \xi}^{(1)}\right)=\sum_{\alpha}(-1)^{\sum_{0<v} \operatorname{dim} N_{v}} \operatorname{Ind}\left(J_{T, \xi}^{-1} D_{T, \xi, \alpha}^{(1)} J_{T, \xi}\right) \\
& =\sum_{\alpha}(-1)^{\sum_{0<v} \operatorname{dim} N_{v}} \operatorname{Ind}\left(D_{\xi}^{Y_{\alpha}}\right) \quad \text { in } \quad K(B) .
\end{aligned}
$$

By (1.41), (1.42) and (1.43), one deduces the first equation of (1.17) easily.

To get the second equation of (1.17), we only need to apply the first equation of (1.17) to the $S^{1}$-action on $M$ defined by the inverse of the original $S^{1}$-action on $M$. The proof of Theorem 1.1 is complete. 


\subsection{The $\operatorname{Spin}^{c}$ case.}

We will keep the notations in Section 1.1. For future applications, in this subsection, we will extend Theorem 1.1 to $\operatorname{Spin}^{c}$ cases.

Let $\pi: M \rightarrow B$ be a fibration of compact manifolds with compact fiber $X$ such that $\operatorname{dim} X=2 l$ and $S^{1}$ acts fiberwise on $M$. Let $h^{T X}$ be a metric on $T X$. We assume that $T X$ is oriented. Let $\left(W, h^{W}\right)$ be an Hermitian complex vector bundle over $M$.

Let $V$ be a $2 p$ dimensional oriented real vector bundle over $M$. Let $L$ be a complex line bundle over $M$ with the property that the vector bundle $U=T X \oplus V$ satisfies $w_{2}(U)=c_{1}(L) \bmod (2)$ where $w_{2}$ denotes the second Stiefel-Whitney class, and $c_{1}(L)$ is the first Chern class of $L$. Then the vector bundle $U$ has a $\operatorname{Spin}^{c}$-structure. Let $h^{V}, h^{L}$ be metrics on $V, L$. Let $S(U, L)$ be the fundamental complex spinor bundle for $(U, L)$ [LaM, Appendix D.9].

Assume that the $S^{1}$-action on $M$ lifts to $V, L$ and $W$, and assume the metrics $h^{T X}, h^{V}, h^{L}, h^{W}$ are $S^{1}$-invariant. Also assume that the $S^{1}$-actions on $T X, V, L$ lift to $S(U, L)$.

Let $\nabla^{T X}$ be the Levi-Civita connection on $\left(T X, h^{T X}\right)$ along the fiber $X$. Let $\nabla^{V}, \nabla^{L}$ and $\nabla^{W}$ be $S^{1}$ invariant and metric-compatible connections on $\left(V, h^{V}\right),\left(L, h^{L}\right)$ and $\left(W, h^{W}\right)$ respectively. Let $\nabla^{S(U, L)}$ be the Hermitian connection on $S(U, L)$ induced by $\nabla^{T X} \oplus \nabla^{V}$ and $\nabla^{L}$ as in Section 1.1. Let $\nabla^{S(U, L) \otimes W}$ be the tensor product connection on $S(U, L) \otimes W$ induced by $\nabla^{S(U, L)}$ and $\nabla^{W}$.

Let $\left\{e_{i}\right\}_{i=1}^{2 l},\left\{f_{j}\right\}_{j=1}^{2 p}$ be the corresponding oriented orthonormal bases of $\left(T X, h^{T X}\right)$ and $\left(V, h^{V}\right)$. Let $D^{X}$ be the family $\operatorname{Spin}^{c}$-Dirac operator on the fiber $X$,

$$
D^{X}=\sum_{i=1}^{2 l} c\left(e_{i}\right) \nabla_{e_{i}}^{S(U, L) \otimes W} .
$$

There are two canonical ways to consider $S(U, L)$ as a $\mathbf{Z}_{2}$-graded vector bundle. Let

$$
\begin{aligned}
\tau_{s} & =i^{l} c\left(e_{1}\right) \cdots c\left(e_{2 l}\right), \\
\tau_{e} & =i^{l+p} c\left(e_{1}\right) \cdots c\left(e_{2 l}\right) c\left(f_{1}\right) \cdots c\left(f_{2 p}\right),
\end{aligned}
$$

be two involutions of $S(U, L)$. Then $\tau_{s}^{2}=\tau_{e}^{2}=1$. We decompose $S(U, L)=$ $S^{+}(U, L) \oplus S^{-}(U, L)$ corresponding to $\tau_{s}$ (resp. $\left.\tau_{e}\right)$ such that $\left.\tau_{s}\right|_{S^{ \pm}(U, L)}= \pm 1$ (resp. $\left.\tau_{e}\right|_{S^{ \pm}(U, L)}= \pm 1$ ). For $\tau=\tau_{s}$ or $\tau_{e}$, we can define the index bundle $\operatorname{Ind}_{\tau}\left(D^{X}\right) \in K_{S^{1}}(B)$ as in Section 1.1. 
We have the following $S^{1}$-equivariant decomposition of $V$ restricted to $F$,

$$
\left.V\right|_{F}=\oplus_{v \neq 0} V_{v} \oplus V_{0}^{\mathbf{R}},
$$

where $V_{v}$ is a complex vector bundle such that $g$ acts on it by $g^{v}$, and $V_{0}^{\mathbf{R}}$ is the real subbundle of $V$ such that $S^{1}$ acts as identity. For $v \neq 0$, let $V_{v, \mathbf{R}}$ denote the underlying real vector bundle of $V_{v}$. Denote by $2 p^{\prime}=\operatorname{dim} V_{0}^{\mathbf{R}}$ and $2 l^{\prime}=\operatorname{dim} Y$. Let us write

$$
L_{F}=L \otimes\left(\bigotimes_{v \neq 0} \operatorname{det} N_{v} \otimes \bigotimes_{v \neq 0} \operatorname{det} V_{v}\right)^{-1} .
$$

Then $T Y \oplus V_{0}^{\mathbf{R}}$ has a $\operatorname{Spin}^{c}$ structure since $w_{2}\left(T Y \oplus V_{0}^{\mathbf{R}}\right)=c_{1}\left(L_{F}\right) \bmod (2)$. Let $S\left(T Y \oplus V_{0}^{\mathbf{R}}, L_{F}\right)$ be the fundamental spinor bundle for $\left(T Y \oplus V_{0}^{\mathbf{R}}, L_{F}\right)$.

Let $D^{Y}, D^{Y_{\alpha}}$ be the families of $\operatorname{Spin}^{c}$ Dirac operators acting on $S(T Y \oplus$ $\left.V_{0}^{\mathbf{R}}, L_{F}\right)$ over $F, F_{\alpha}$. If $R$ is an Hermitian complex vector bundle equipped with an Hermitian connection over $F$, let $D^{Y} \otimes R, D^{Y_{\alpha}} \otimes R$ denote the twisted Spin ${ }^{c}$ Dirac operators on $S\left(T Y \oplus V_{0}^{\mathbf{R}}, L_{F}\right) \otimes R$.

Recall that $N_{v, \mathbf{R}}$ and $V_{v, \mathbf{R}}$ are canonically oriented by their complex structures. The decompositions (1.8), (1.46) induce the orientations of $T Y$ and $V_{0}^{\mathbf{R}}$. Let $\left\{e_{i}\right\}_{i=1}^{2 l^{\prime}},\left\{f_{j}\right\}_{j=1}^{2 p^{\prime}}$ be the corresponding oriented orthonormal basis of $\left(T Y, h^{T Y}\right)$ and $\left(V_{0}^{\mathbf{R}}, h^{V_{0}^{\mathbf{R}}}\right)$. There are two canonical ways to consider $S\left(T Y \oplus V_{0}^{\mathbf{R}}, L_{F}\right)$ as a $\mathbf{Z}_{2}$-graded vector bundle: let

$$
\begin{aligned}
\tau_{s} & =i^{l^{\prime}} c\left(e_{1}\right) \cdots c\left(e_{2 l^{\prime}}\right), \\
\tau_{e} & =i^{l^{\prime}+p^{\prime}} c\left(e_{1}\right) \cdots c\left(e_{2 l^{\prime}}\right) c\left(f_{1}\right) \cdots c\left(f_{2 p^{\prime}}\right),
\end{aligned}
$$

be two involutions of $S\left(T Y \oplus V_{0}^{\mathbf{R}}, L_{F}\right)$. Then $\tau_{s}^{2}=\tau_{e}^{2}=1$. We decompose $S\left(T Y \oplus V_{0}^{\mathbf{R}}, L_{F}\right)=S^{+}\left(T Y \oplus V_{0}^{\mathbf{R}}, L_{F}\right) \oplus S^{-}\left(T Y \oplus V_{0}^{\mathbf{R}}, L_{F}\right)$ corresponding to $\tau_{s}$ (resp. $\tau_{e}$ ) such that $\left.\tau_{s}\right|_{S^{ \pm}\left(T Y \oplus V_{0}^{\mathbf{R}}, L_{F}\right)}= \pm 1$ (resp. $\left.\tau_{e}\right|_{S^{ \pm}\left(T Y \oplus V_{0}^{\mathbf{R}}, L_{F}\right)}= \pm 1$ ).

By restricting to $F$, one has the following isomorphism of $\mathbf{Z}_{2}$-graded Clifford modules over $F$,

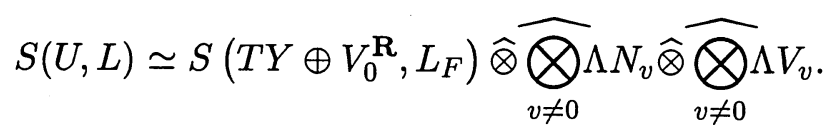

Here we denote the $\mathbf{Z}_{2}$-graded tensor product by $\widehat{\otimes}$ (cf. [LaM, p. 11]). We denote by $\operatorname{Ind}_{\tau_{s}}, \operatorname{Ind}_{\tau_{e}}$ the index bundles corresponding to the involutions $\tau_{s}, \tau_{e}$. 
Let $S^{1}$ act on $L$ by send $g \in S^{1}$ to $g^{l_{c}}\left(l_{c} \in \mathbf{Z}\right)$ on $F$. Then $l_{c}$ is locally constant on $F$. We define the following elements in $K(F)\left[\left[q^{1 / 2}\right]\right],{ }^{4}$

$$
\begin{array}{r}
R_{ \pm}(q)=q^{\frac{1}{2} \sum_{v}|v| \operatorname{dim} N_{v}-\frac{1}{2} \sum_{v} v \operatorname{dim} V_{v}+\frac{1}{2} l_{c}} \bigotimes_{0<v}\left(\operatorname{Sym}_{q^{v}}\left(N_{v}\right) \otimes \operatorname{det} N_{v}\right) \\
\otimes \bigotimes_{v<0} \operatorname{Sym}_{q^{-v}}\left(\bar{N}_{v}\right) \otimes \bigotimes_{v \neq 0} \Lambda_{ \pm q^{v}}\left(V_{v}\right) \otimes\left(\sum_{v} q^{v} W_{v}\right)=\sum_{n} R_{ \pm, n} q^{n} \\
R_{ \pm}^{\prime}(q)=q^{-\frac{1}{2} \sum_{v}|v| \operatorname{dim} N_{v}-\frac{1}{2} \sum_{v} v \operatorname{dim} V_{v}+\frac{1}{2} l_{c}} \bigotimes_{0<v} \operatorname{Sym}_{q^{-v}}\left(\bar{N}_{v}\right) \\
\otimes \bigotimes_{v<0}\left(\operatorname{Sym}_{q^{v}}\left(N_{v}\right) \otimes \operatorname{det} N_{v}\right) \otimes \bigotimes_{v \neq 0} \Lambda_{ \pm q^{v}}\left(V_{v}\right) \otimes\left(\sum_{v} q^{v} W_{v}\right)=\sum_{n} R_{ \pm, n}^{\prime} q^{n}
\end{array}
$$

As $T X \oplus V \oplus L$ is spin, and the $S^{1}$ action lifts to the spinor bundle of $U \oplus L$, by using the same argument as (3.30) below, we know that

$$
\sum_{v} v \operatorname{dim} N_{v}+\sum_{v} v \operatorname{dim} V_{v}+l_{c}=0 \bmod (2)
$$

The following result generalizes [T, Theorem 2.6] to the family case.

Theorem 1.2. For $n \in \mathbf{Z}$, we have the following identities in $K(B)$ :

$$
\begin{array}{r}
\operatorname{Ind}_{\tau_{s}}\left(D^{X}, n\right)=\sum_{\alpha}(-1)^{\sum_{0<v} \operatorname{dim} N_{v}} \operatorname{Ind}_{\tau_{s}}\left(D^{Y_{\alpha}} \otimes R_{+, n}\right) \\
=\sum_{\alpha}(-1)^{\sum_{v<0} \operatorname{dim} N_{v}} \operatorname{Ind}_{\tau_{s}}\left(D^{Y_{\alpha}} \otimes R_{+, n}^{\prime}\right), \\
\operatorname{Ind}_{\tau_{e}}\left(D^{X}, n\right)=\sum_{\alpha}(-1)^{\sum_{0<v} \operatorname{dim} N_{v}} \operatorname{Ind}_{\tau_{e}}\left(D^{Y_{\alpha}} \otimes R_{-, n}\right) \\
=\sum_{\alpha}(-1)^{\sum_{v<0} \operatorname{dim} N_{v}} \operatorname{Ind}_{\tau_{e}}\left(D^{Y_{\alpha}} \otimes R_{-, n}^{\prime}\right) .
\end{array}
$$

Proof: The proof is a straightforward generalization of the proof of Theorem 1.1. The details are left to the interested reader.

\footnotetext{
${ }^{4}$ There is a misprint in [LMZ2] , we should replace $\otimes_{v} q^{v} W_{v}$ in (1.11) of [LMZ2] by $\otimes\left(\sum_{v} q^{v} W_{v}\right)$.
} 


\section{Family rigidity and vanishing theorems.}

The purpose of this section is to establish the main results of this paper: the rigidity and vanishing theorems on the equivariant $K$-theory level. The results in this section refine some of the results in $[\mathbf{L M 1}, 2]$ to the $K$-theory level.

As in the previous sections, we let $\pi: M \rightarrow B$ be a fibration of compact manifolds with fiber $X$. We assume that $S^{1}$ acts fiberwise on $M$ with fixed point set $F$, and $T X$ has an $S^{1}$-equivariant spin structure. Then $\pi: F \rightarrow B$ is a fibration with fiber $Y$.

Following Witten, we will introduce some elements $R(q)=\Sigma_{n \in \mathrm{N}} q^{n} R_{n} \in$ $K_{S^{1}}(M)[[q]]$. To prove the rigidity theorem for these elements, Taubes and Witten suggested to use some shift operators to get a relation like $\operatorname{Ind}\left(D^{X} \otimes\right.$ $\left.R_{m}, h\right)=\operatorname{Ind}\left(D^{X} \otimes R_{m+h}, h\right)$ for $h, m \in \mathbf{Z}$. As $R_{m}=0$ for $m<0$, this implies the rigidity theorem. See the paper of Taubes [T] for a rigorous treatment.

To get a similar equality in the family case, we first apply our $K$-theory version of the equivariant family index theorem [LM1, Theorem 1.1], Theorem 1.1, to reduce the problem to the fixed point set $F$. Then we introduce an auxiliary element in $K_{S^{1}}(F)[[q]]$. We study the corresponding index bundles of the twisted $\operatorname{Spin}^{c}$ Dirac operators on $Y$, which, after doing some shift operations, will be related to a term like $\operatorname{Ind}\left(D^{X} \otimes R_{m+h}, h\right)$. On the other hand, if we apply our $K$-theory version of the equivariant family index theorem iteratively, we may also relate the considered index bundle to a term like $\operatorname{Ind}\left(D^{X} \otimes R_{m}, h\right)$. This then completes the proof. To apply the equivariant family index theorem, we are inspired by the constructions of Taubes [T, $\S 6]$. Namely, we will construct some operators on the fixed point set $M(n)$ of the induced $\mathbf{Z}_{n}$-action on $M$, and apply the equivariant family index theorem to them.

As was pointed out in the introduction, our main observation is that we can directly construct and apply the shift operators on the fixed point set. In this way, we avoid the use of the Dirac operators on the normal bundle in the loop space and the associated analysis of Fredholm properties of these operators in $[\mathbf{T}]$.

This section is organized as follows: In Section 2.1, we state our main results, the rigidity and vanishing theorems on the equivariant $K$-theory level. In Section 2.2, we state two intermediate results which will be used to prove our main results stated in Section 2.1. In Section 2.3, we prove the family rigidity and vanishing theorem. In Section 2.4 , we prove a vanishing theorem on the equivariant $K$-theory level of the index bundle of the Dirac 
operator on loop spaces, which may be viewed as a loop space analogue of Corollary 1.1, and which also extends the corresponding loop space analogue of the Atiyah-Hirzebruch theorem in [Liu2] to the family case.

Throughout this section, we use the notations of Section 1.1.

\subsection{Family rigidity and vanishing theorems.}

Let $\pi: M \rightarrow B$ be a fibration of compact manifolds with fiber $X$ and $\operatorname{dim} X=2 l$. We assume that $S^{1}$ acts fiberwise on $M$, and $T X$ has an $S^{1-}$ invariant spin structure. As in [AH], by lifting to the double cover of $S^{1}$, we can assume that the second condition is always satisfied. Let $V$ be a real vector bundle over $M$ with structure group $\operatorname{Spin}(2 k)$. We assume that $V$ has an $S^{1}$-invariant spin structure. Let $S(V)=S^{+}(V) \oplus S^{-}(V)$ be the spinor bundle of $V$.

The purpose of this section is to prove that the elliptic operators introduced by Witten [W] have some interesting rigidity and vanishing properties on the equivariant $K$-theory level. Let us recall some definitions first.

For a complex (resp. real) vector bundle $E$ over $M$, let

$$
\begin{aligned}
& \operatorname{Sym}_{t}(E)=1+t E+t^{2} \operatorname{Sym}^{2} E+\cdots, \\
& \Lambda_{t}(E)=1+t E+t^{2} \Lambda^{2} E+\cdots
\end{aligned}
$$

be the symmetric and exterior power operations of $E$ (resp. $E \otimes_{\mathbf{R}} \mathbf{C}$ ) in $K(M)[[t]]$ respectively. Set

$$
\begin{aligned}
& \Theta_{q}^{\prime}(T X)=\bigotimes_{n=1}^{\infty} \Lambda_{q^{n}}(T X) \otimes \bigotimes_{n=1}^{\infty} \operatorname{Sym}_{q^{n}}(T X), \\
& \Theta_{q}(T X)=\bigotimes_{n=1}^{\infty} \Lambda_{-q^{n-1 / 2}}(T X) \otimes \bigotimes_{n=1}^{\infty} \operatorname{Sym}_{q^{n}}(T X), \\
& \Theta_{-q}(T X)=\bigotimes_{n=1}^{\infty} \Lambda_{q^{n-1 / 2}}(T X) \otimes \bigotimes_{n=1}^{\infty} \operatorname{Sym}_{q^{n}}(T X) .
\end{aligned}
$$


We also define the following elements in $K(M)\left[\left[q^{1 / 2}\right]\right]$ :

$$
\begin{aligned}
& \Theta_{q}^{\prime}(T X \mid V)=\bigotimes_{n=1}^{\infty} \Lambda_{q^{n}}(V) \otimes \bigotimes_{n=1}^{\infty} \operatorname{Sym}_{q^{n}}(T X), \\
& \Theta_{q}(T X \mid V)=\bigotimes_{n=1}^{\infty} \Lambda_{-q^{n-1 / 2}}(V) \otimes \bigotimes_{n=1}^{\infty} \operatorname{Sym}_{q^{n}}(T X), \\
& \Theta_{-q}(T X \mid V)=\bigotimes_{n=1}^{\infty} \Lambda_{q^{n-1 / 2}}(V) \otimes \bigotimes_{n=1}^{\infty} \operatorname{Sym}_{q^{n}}(T X), \\
& \Theta_{q}^{*}(T X \mid V)=\bigotimes_{n=1}^{\infty} \Lambda_{-q^{n}}(V) \otimes \bigotimes_{n=1}^{\infty} \operatorname{Sym}_{q^{n}}(T X) .
\end{aligned}
$$

Recall that the equivariant cohomology group $H_{S^{1}}^{*}(M, \mathbf{Z})$ of $M$ is defined by

$$
H_{S^{1}}^{*}(M, \mathbf{Z})=H^{*}\left(M \times_{S^{1}} E S^{1}, \mathbf{Z}\right)
$$

where $E S^{1}$ is the universal $S^{1}$-principal bundle over the classifying space $B S^{1}$ of $S^{1}$. So $H_{S^{1}}^{*}(M, \mathbf{Z})$ is a module over $H^{*}\left(B S^{1}, \mathbf{Z}\right)$ induced by the projection $\bar{\pi}: M \times_{S^{1}} E S^{1} \rightarrow B S^{1}$. Let $p_{1}(V)_{S^{1}}, p_{1}(T X)_{S^{1}} \in H_{S^{1}}^{*}(M, \mathbf{Z})$ be the equivariant first Pontrjagin classes of $V$ and $T X$ respectively. As $V \times{ }_{S^{1}} E S^{1}$ and $T X \times_{S^{1}} E S^{1}$ are spin over $M \times_{S^{1}} E S^{1}$, one knows that $\frac{1}{2} p_{1}(V)_{S^{1}}$ and $\frac{1}{2} p_{1}(T X)_{S^{1}}$ are well-defined in $H_{S^{1}}^{*}(M, \mathbf{Z})$ (cf. [T, pp. 456457]). Also recall that

$$
H^{*}\left(B S^{1}, \mathbf{Z}\right)=\mathbf{Z}[u]
$$

with $u$ a generator of degree 2 .

In the following, we denote by $D^{X} \otimes W$ the family of Dirac operators acting fiberwise on $S(T X) \otimes W$ as defined in Section 1.1. We also write $d_{s}^{X}=D^{X} \otimes S(T X)$.

We can now state the main results of this paper as follows. The first one is a family generalization of the Witten rigidity theorems as proved in [ $\mathbf{T}]$, [BT] and [Liu1]. It also refines [ LM1, Theorem 2.1].

Theorem 2.1. (a) The family operators $d_{s}^{X} \otimes \Theta_{q}^{\prime}(T X), D^{X} \otimes \Theta_{q}(T X)$ and $D^{X} \otimes \Theta_{-q}(T X)$ are rigid on the equivariant $K$-theory level.

(b) If $\frac{1}{2} p_{1}(V)_{S^{1}}=\frac{1}{2} p_{1}(T X)_{S^{1}}$, then $D^{X} \otimes\left(S^{+}(V)+S^{-}(V)\right) \otimes \Theta_{q}^{\prime}(T X \mid V)$, $D^{X} \otimes\left(S^{+}(V)-S^{-}(V)\right) \otimes \Theta_{q}^{*}(T X \mid V), D^{X} \otimes \Theta_{q}(T X \mid V)$ and $D^{X} \otimes \Theta_{-q}(T X \mid V)$ are rigid on the equivariant $K$-theory level. 
The second main result generalizes the vanishing results of Taubes [T, Proposition 10.1] and Liu [Liu2, Corollary 3.3] to the family case. It also refines a result in [LM1, Theorem 3.2].

Theorem 2.2. If $\frac{1}{2} p_{1}(V)_{S^{1}}-\frac{1}{2} p_{1}(T X)_{S^{1}}=e \cdot \bar{\pi}^{*} u^{2}$ with $e \in \mathbf{Z}$ satisfying $e<0$, then the index bundles of $D^{X} \otimes\left(S^{+}(V)+S^{-}(V)\right) \otimes \Theta_{q}^{\prime}(T X \mid V)$, $D^{X} \otimes\left(S^{+}(V)-S^{-}(V)\right) \otimes \Theta_{q}^{*}(T X \mid V), D^{X} \otimes \Theta_{q}(T X \mid V)$ and $D^{X} \otimes \Theta_{-q}(T X \mid V)$ are zero in $K_{S^{1}}(B)$. In particular, they are identically zero in $K(B)$.

A quite interesting consequence of the above results is the following family $\widehat{A}$-vanishing theorem for loop spaces. It extends the corresponding loop space analogue of the Atiyah-Hirzebruch theorem in [Liu2, Theorem 6] to the family case.

Theorem 2.3. Assume $M$ is connected and the $S^{1}$-action is nontrivial. If $\frac{1}{2} p_{1}(T X)_{S^{1}}=-e \cdot \bar{\pi}^{*} u^{2}$ for some integer $e$, then the index bundle of $D^{X} \otimes$ $\bigotimes_{n=1}^{\infty} \operatorname{Sym}_{q^{n}}(T X)$ as an element in $K_{S^{1}}(B)$, in particular, as an element in $K(B)$, is identically zero.

As was pointed out by Dessai [De], when the $S^{1}$-action is induced from a fiberwise $S^{3}$ action on $M$ which preserves the spin structure of $T X$, the condition $\frac{1}{2} p_{1}(T X)_{S^{1}}=-e \cdot \bar{\pi}^{*} u^{2}$ in $H_{S^{1}}^{*}(M, \mathbf{Z})$ is equivalent to $\frac{1}{2} p_{1}(T X)=0$ in $H^{*}(M, \mathbf{Z})$. So one gets the following family vanishing theorem.

Corollary 2.1. Assume $M$ is connected and admits a nontrivial $S^{1}$ action induced by a fiberwise $S^{3}$-action which preserves the spin structure of $T X$. If $\frac{1}{2} p_{1}(T X)=0$, then the index bundle as an element in $K_{S^{1}}(B)$ (in particular, as an element in $K(B)$ ), of $D^{X} \otimes \bigotimes_{n=1}^{\infty} \operatorname{Sym}_{q^{n}}(T X)$, is identically zero.

Actually, our proof of these theorems works under the following slightly weaker hypothesis. Let us first explain some notations.

For each $n>1$, consider $\mathbf{Z}_{n} \subset S^{1}$, the cyclic subgroup of order $n$. We have the $\mathbf{Z}_{n}$ equivariant cohomology of $M$ defined by $H_{\mathbf{Z}_{n}}^{*}(M, \mathbf{Z})=$ $H^{*}\left(M \times \mathbf{z}_{n} E S^{1}, \mathbf{Z}\right)$, and there is a natural "forgetful" map $\alpha\left(S^{1}, \mathbf{Z}_{n}\right): M \times \mathbf{z}_{n}$ $E S^{1} \rightarrow M \times_{S^{1}} E S^{1}$ which induces a pullback $\alpha\left(S^{1}, \mathbf{Z}_{n}\right)^{*}: H_{S^{1}}^{*}(M, \mathbf{Z}) \rightarrow$ $H_{\mathbf{Z}_{n}}^{*}(M, \mathbf{Z})$. The arrow which forgets the $S^{1}$ action altogether we denote by $\alpha\left(S^{1}, 1\right)$. Thus $\alpha\left(S^{1}, 1\right)^{*}: H_{S^{1}}^{*}(M, \mathbf{Z}) \rightarrow H^{*}(M, \mathbf{Z})$ is induced by the inclusion of $M$ into $M \times_{S^{1}} E S^{1}$ as a fiber over $B S^{1}$.

Finally, note that, if $\mathbf{Z}_{n}$ acts trivially on a space $Y$, then there is another map $r^{*}: H^{*}(Y, \mathbf{Z}) \rightarrow H_{\mathbf{Z}_{n}}^{*}(Y, \mathbf{Z})$ induced by the projection $Y \times \mathbf{Z}_{n} E S^{1}=$ $Y \times B \mathbf{Z}_{n} \stackrel{r}{\rightarrow} Y$. 
We let $\mathbf{Z}_{\infty}=S^{1}$. For each $1<n \leq+\infty$, let $i: M(n) \rightarrow M$ be the inclusion of the fixed point set of $\mathbf{Z}_{n} \subset S^{1}$ in $M$, and so $i$ induces $i_{S^{1}}: M(n) \times_{S^{1}} E S^{1} \rightarrow M \times_{S^{1}} E S^{1}$.

In the rest of this paper, we suppose that there exists some integer $e \in \mathbf{Z}$ such that for each $1<n \leq+\infty$,

$$
\begin{aligned}
\alpha\left(S^{1}, \mathbf{z}_{n}\right)^{*} \circ i_{S^{1}}^{*}\left(\frac{1}{2} p_{1}(V-T X)_{S^{1}}-e \cdot \bar{\pi}^{*} u^{2}\right) \\
=r^{*} \circ \alpha\left(S^{1}, 1\right)^{*} \circ i_{S^{1}}^{*}\left(\frac{1}{2} p_{1}(V-T X)_{S^{1}}\right)
\end{aligned}
$$

Remark 2.1. The relation (2.6) clearly follows from the hypothesis of Theorems 2.1 and 2.2 by pulling back and forgetting. Thus it is a weaker condition.

Remark 2.2. If $e=0$, and $B$ is a point, (2.6) is exactly [BT, (11.11)].

We can now state a slightly more general version of Theorems 2.1 and 2.2 .

Theorem 2.4. Under the hypothesis (2.6), we have

i) If $e=0$, then the index bundles of the elliptic operators in Theorem 2.1(b) are rigid on the equivariant $\mathrm{K}$-theory level.

ii) If $e<0$, then the index bundles of the elliptic operators in Theorem 2.1(b) are zero as elements in $K_{S^{1}}(B)$. In particular, these index bundles are zero in $K(B)$.

The rest of this section is devoted to a proof of Theorem 2.4.

\subsection{Two intermediate results.}

Let $F=\left\{F_{\alpha}\right\}$ be the fixed point set of the circle action. Then $\pi: F \rightarrow B$ is a fibration with compact fiber denoted by $Y=\left\{Y_{\alpha}\right\}$. Recall that

$$
\left.T X\right|_{F}=T Y \oplus \bigoplus_{v \neq 0} N_{v, \mathbf{R}}
$$

where $N_{v, \mathbf{R}}$ denotes the underlying real bundle of the complex vector bundle $N_{v}$ on which $g \in S^{1}$ acts by multiplying by $g^{v}$. Since we can choose either $N_{v}$ or $\bar{N}_{v}$ as the complex vector bundle for $N_{v, \mathbf{R}}$, in what follows we may 
and we will assume that

$$
\begin{aligned}
& \left.T X\right|_{F}=T Y \oplus \bigoplus_{0<v} N_{v} \\
& \left.T X \otimes_{\mathbf{R}} \mathbf{C}\right|_{F}=\left(T Y \otimes_{\mathbf{R}} \mathbf{C}\right) \oplus \bigoplus_{0<v}\left(N_{v} \oplus \bar{N}_{v}\right)
\end{aligned}
$$

where $N_{v}$ is the complex vector bundle on which $g \in S^{1}$ acts by multiplying by $g^{v}$. Here some $N_{v}$ may be zero. Similarly, let

$$
\left.V\right|_{F}=V_{0}^{\mathbf{R}} \oplus \bigoplus_{0<v} V_{v},
$$

where $V_{v}$ is a complex vector bundle on which $g \in S^{1}$ acts by multiplying by $g^{v}$, and $V_{0}^{\mathbf{R}}$ is a real vector bundle on which $S^{1}$ acts as identity.

On $F$, let

$$
\begin{array}{ll}
e(N)=\sum_{0<v} v^{2} \operatorname{dim} N_{v}, & d^{\prime}(N)=\sum_{0<v} v \operatorname{dim} N_{v}, \\
e(V)=\sum_{0<v} v^{2} \operatorname{dim} V_{v}, & d^{\prime}(V)=\sum_{0<v} v \operatorname{dim} V_{v} .
\end{array}
$$

Then $e(N), e(V), d^{\prime}(N)$ and $d^{\prime}(V)$ are locally constant functions on $F$.

Let us introduce some line bundles:

$$
\begin{aligned}
& L(N)=\bigotimes_{0<v}\left(\operatorname{det} N_{v}\right)^{v}, \quad L(V)=\bigotimes_{0<v}\left(\operatorname{det} V_{v}\right)^{v}, \\
& L=L(N)^{-1} \otimes L(V) .
\end{aligned}
$$

We denote the Chern roots of $N_{v}$ by $\left\{x_{v}^{j}\right\}$ (resp. $V_{v}$ by $\left\{u_{v}^{j}\right\}$ ), and the Chern roots of $T Y \otimes_{\mathbf{R}} \mathbf{C}$ by $\left\{ \pm y_{j}\right\}$ (resp. $V_{0}=V_{0}^{\mathbf{R}} \otimes_{\mathbf{R}} \mathbf{C}$ by $\left\{ \pm u_{0}^{j}\right\}$ ). Then if we take $\mathbf{Z}_{\infty}=S^{1}$ in (2.6), we get

$$
\begin{aligned}
& p_{1}(V)_{S^{1}}=\sum_{v, j}\left(u_{v}^{j}+v u\right)^{2} \\
& p_{1}(T X)_{S^{1}}=\sum_{j}\left(y_{j}\right)^{2}+\sum_{v, j}\left(x_{v}^{j}+v u\right)^{2} \\
& \frac{1}{2}\left(\sum_{v, j}\left(u_{v}^{j}+v u\right)^{2}-\sum_{j}\left(y_{j}\right)^{2}-\sum_{v, j}\left(x_{v}^{j}+v u\right)^{2}\right)-e u^{2} \\
& =\frac{1}{2}\left(\sum_{v, j}\left(u_{v}^{j}\right)^{2}-\sum_{j}\left(y_{j}\right)^{2}-\sum_{v, j}\left(x_{v}^{j}\right)^{2}\right) .
\end{aligned}
$$


By (2.5), (2.11), we get

$$
\begin{aligned}
& c_{1}(L)=\sum_{v, j} v u_{v}^{j}-\sum_{v, j} v x_{v}^{j}=0, \\
& e(V)-e(N)=\sum_{0<v} v^{2} \operatorname{dim} V_{v}-\sum_{0<v} v^{2} \operatorname{dim} N_{v}=2 e .
\end{aligned}
$$

This means $L$ is a trivial complex line bundle over each component $F_{\alpha}$ of $F$, and $g \in S^{1}$ acts on $L$ by multiplying by $g^{2 e}$. So we can extend $L$ to a trivial complex line bundle over $M$, and we extend the $S^{1}$ action on it by multiplying the canonical section 1 of $L$ to $g^{2 e} \cdot 1$ for $g \in S^{1}$.

The line bundles in (2.10) will play important roles in the next two sections which contain the proofs of Theorems $2.5,2.6$ to be stated below.

We now define the following elements in $K_{S^{1}}(M)\left[\left[q^{1 / 2}\right]\right]$ :

$$
\begin{aligned}
& R_{1}(V)=\left(S^{+}(V)+S^{-}(V)\right) \otimes \bigotimes_{n=1}^{\infty} \Lambda_{q^{n}}(V), \\
& R_{2}(V)=\left(S^{+}(V)-S^{-}(V)\right) \otimes \bigotimes_{n=1}^{\infty} \Lambda_{-q^{n}}(V), \\
& R_{3}(V)=\bigotimes_{n=1}^{\infty} \Lambda_{-q^{n-1 / 2}}(V), \quad R_{4}(V)=\bigotimes_{n=1}^{\infty} \Lambda_{q^{n-1 / 2}}(V) .
\end{aligned}
$$

In what follows, if $R(q)=\sum_{m \in \frac{1}{2} \mathbf{z}} R_{m} q^{m} \in K_{S^{1}}(M)\left[\left[q^{1 / 2}\right]\right]$, we will also denote $\operatorname{Ind}\left(D^{X} \otimes R_{m}, h\right)$ by $\operatorname{Ind}\left(D^{X} \otimes R(q), m, h\right)$.

We first state a result which expresses the global equivariant family index via the family indices on the fixed point set.

Proposition 2.1. For $m \in \frac{1}{2} \mathbf{Z}, h \in \mathbf{Z}, 1 \leq i \leq 4$, we have the following identity in $K(B)$,

$$
\begin{aligned}
& \text { Ind }\left(D^{X} \otimes \bigotimes_{n=1}^{\infty} \operatorname{Sym}_{q^{n}}(T X) \otimes R_{i}(V), m, h\right) \\
& =\sum_{\alpha}(-1)^{\sum_{0<v} \operatorname{dim} N_{v}} \operatorname{Ind}\left(D^{Y_{\alpha}} \otimes \bigotimes_{n=1}^{\infty} \operatorname{Sym}_{q^{n}}(T X) \otimes R_{i}(V)\right. \\
& \left.\qquad \operatorname{Sym}\left(\bigoplus_{0<v} N_{v}\right) \otimes \bigotimes_{0<v} \operatorname{det} N_{v}, m, h\right)
\end{aligned}
$$


Proof: Proposition 2.1 follows directly from Theorem 1.1.

For $p \in \mathbf{N}$, we define the following elements in $K_{S^{1}}(F)[[q]]:{ }^{5}$

$$
\begin{aligned}
& \mathcal{F}_{p}(X)=\bigotimes_{0<v}\left(\bigotimes_{n=1}^{\infty} \operatorname{Sym}_{q^{n}}\left(N_{v}\right) \otimes \bigotimes_{n>p v} \operatorname{Sym}_{q^{n}}\left(\bar{N}_{v}\right)\right) \otimes \bigotimes_{n=1}^{\infty} \operatorname{Sym}_{q^{n}}(T Y), \\
& \mathcal{F}_{p}^{\prime}(X)=\bigotimes_{\substack{0<v \\
0 \leq n \leq p v}}\left(\operatorname{Sym}_{q^{-n}}\left(N_{v}\right) \otimes \operatorname{det} N_{v}\right) \\
& \mathcal{F}^{-p}(X)=\mathcal{F}_{p}(X) \otimes \mathcal{F}_{p}^{\prime}(X) .
\end{aligned}
$$

Then, by (2.7), over $F$, we have

$$
\mathcal{F}^{0}(X)=\bigotimes_{n=1}^{\infty} \operatorname{Sym}_{q^{n}}(T X) \otimes \operatorname{Sym}\left(\bigoplus_{0<v} N_{v}\right) \otimes \bigotimes_{0<v} \operatorname{det} N_{v} .
$$

We now state two intermediate results on the relations between the family indices on the fixed point set. They will be used in the next subsection to prove Theorem 2.4 .

Theorem 2.5. For $1 \leq i \leq 4, h, p \in \mathbf{Z}$ and $p>0, m \in \frac{1}{2} \mathbf{Z}$, we have

$$
\begin{aligned}
& \sum_{\alpha}(-1)^{\sum_{0<v} \operatorname{dim} N_{v}} \operatorname{Ind}\left(D^{Y_{\alpha}} \otimes \mathcal{F}^{0}(X) \otimes R_{i}(V), m, h\right) \\
&=\sum_{\alpha}(-1)^{\sum_{0<v} \operatorname{dim} N_{v}} \operatorname{Ind}\left(D^{Y_{\alpha}} \otimes \mathcal{F}^{-p}(X) \otimes R_{i}(V)\right. \\
&\left.m+\frac{1}{2} p^{2} e(N)+\frac{1}{2} p d^{\prime}(N), h\right)
\end{aligned}
$$

Theorem 2.6. For each $\alpha, 1 \leq i \leq 4, h, p \in \mathbf{Z}, p>0, m \in \frac{1}{2} \mathbf{Z}$, we have the following identity in $K(B)$ :

$$
\begin{aligned}
\operatorname{Ind}\left(D^{Y_{\alpha}}\right. & \left.\otimes \mathcal{F}^{-p}(X) \otimes R_{i}(V), m+\frac{1}{2} p^{2} e(N)+\frac{1}{2} p d^{\prime}(N), h\right) \\
& =\operatorname{Ind}\left(D^{Y_{\alpha}} \otimes \mathcal{F}^{0}(X) \otimes R_{i}(V), m+p h+p^{2} e, h+2 p e\right)
\end{aligned}
$$

${ }^{5}$ Here by $K_{S^{1}}(F)$ we also mean the direct sum of the form $\oplus_{n \in \mathbf{Z}} E_{n}$ with each $E_{n}$ a finite dimensional vector bundle over $F$ of weight $n$ under the $S^{1}$-action. 
Theorem 2.5 is a direct consequence of Theorem 2.7 to be stated below, which will be proved in Section 4, while Theorem 2.6 will be proved in Section 3.

To state Theorem 2.7, set $J=\left\{v \in \mathbf{N} \mid\right.$ there exists $\alpha$ such that $N_{v} \neq 0$ on $\left.F_{\alpha}\right\}$ and

$$
\Phi=\{\beta \in] 0,1] \mid \text { there exists } v \in J \text { such that } \beta v \in \mathbf{Z}\} .
$$

We order the elements in $\Phi$ so that $\Phi=\left\{\beta_{i} \mid 1 \leq i \leq J_{0}, J_{0} \in \mathbf{N}\right.$ and $\beta_{i}<$ $\left.\beta_{i+1}\right\}$. Then for any integer $1 \leq i \leq J_{0}$, there exist $p_{i}, n_{i} \in \mathbf{N}, 0<p_{i} \leq n_{i}$ with $\left(p_{i}, n_{i}\right)=1$ such that

$$
\beta_{i}=p_{i} / n_{i} .
$$

Clearly, $\beta_{J_{0}}=1$. We also set $p_{0}=0$ and $\beta_{0}=0$.

For $1 \leq j \leq J_{0}, p \in \mathbf{N}^{*}=\mathbf{N} \backslash\{0\}$, set

$$
\begin{aligned}
I_{0}^{p} & =\phi, \quad \text { the empty set, } \\
I_{j}^{p} & =\left\{(v, n) \mid v \in J,(p-1) v<n \leq p v, \frac{n}{v}=p-1+\frac{p_{j}}{n_{j}}\right\}, \\
\bar{I}_{j}^{p} & =\left\{(v, n) \mid v \in J,(p-1) v<n \leq p v, \frac{n}{v}>p-1+\frac{p_{j}}{n_{j}}\right\} .
\end{aligned}
$$

For $0 \leq j \leq J_{0}$, we write

$$
\begin{aligned}
\mathcal{F}_{p, j}(X) & =\mathcal{F}_{p}(X) \otimes \mathcal{F}_{p-1}^{\prime}(X) \\
\otimes & \bigotimes_{(v, n) \in \cup_{i=1}^{j} I_{i}^{p}}\left(\operatorname{Sym}_{q^{-n}}\left(N_{v}\right) \otimes \operatorname{det} N_{v}\right) \otimes \bigotimes_{(v, n) \in \bar{I}_{j}^{p}} \operatorname{Sym}_{q^{n}}\left(\bar{N}_{v}\right) .
\end{aligned}
$$

Then

$$
\mathcal{F}_{p, 0}(X)=\mathcal{F}^{-p+1}(X), \quad \mathcal{F}_{p, J_{0}}(X)=\mathcal{F}^{-p}(X) .
$$

For $s \in \mathbf{R}$, let $[s]$ denote the greatest integer which is less than or equal to the given number $s$. Set

$$
\begin{aligned}
& e\left(p, \beta_{j}, N\right)=\frac{1}{2} \sum_{0<v}\left(\operatorname{dim} N_{v}\right)\left((p-1) v+\left[\frac{p_{j} v}{n_{j}}\right]\right)\left((p-1) v+\left[\frac{p_{j} v}{n_{j}}\right]+1\right), \\
& d_{c}^{\prime}\left(\beta_{j}, N\right)=\sum_{0<v}\left(\operatorname{dim} N_{v}\right)\left[\frac{p_{j} v}{n_{j}}\right] .
\end{aligned}
$$


Then $e\left(p, \beta_{j}, N\right), d^{\prime}\left(\beta_{j}, N\right)$ are locally constant functions on $F$. And

$$
\begin{aligned}
& e\left(p, \beta_{0}, N\right)=\frac{1}{2}(p-1)^{2} e(N)+\frac{1}{2}(p-1) d^{\prime}(N), \\
& e\left(p, \beta_{J_{0}}, N\right)=\frac{1}{2} p^{2} e(N)+\frac{1}{2} p d^{\prime}(N) .
\end{aligned}
$$

Theorem 2.7. For $1 \leq i \leq 4,1 \leq j \leq J_{0}, p \in \mathbf{N}^{*}, h \in \mathbf{Z}, m \in \frac{1}{2} \mathbf{Z}$, we have

$$
\begin{array}{r}
\sum_{\alpha}(-1)^{d^{\prime}\left(\beta_{j-1}, N\right)+\sum_{0<v} \operatorname{dim} N_{v}} \operatorname{Ind}\left(D^{Y_{\alpha}} \otimes \mathcal{F}_{p, j-1}(X) \otimes R_{i}(V),\right. \\
\left.m+e\left(p, \beta_{j-1}, N\right), h\right) \\
=\sum_{\alpha}(-1)^{d^{\prime}\left(\beta_{j}, N\right)+\sum_{0<v} \operatorname{dim} N_{v}} \operatorname{Ind}\left(D^{Y_{\alpha}} \otimes \mathcal{F}_{p, j}(X) \otimes R_{i}(V),\right. \\
\left.m+e\left(p, \beta_{j}, N\right), h\right) .
\end{array}
$$

Proof: The proof is given in Section 4.

Proof of Theorem 2.5: From (2.23), (2.25), and Theorem 2.7, for $1 \leq$ $i \leq 4, h \in \mathbf{Z}, p \in \mathbf{N}^{*}$ and $m \in \frac{1}{2} \mathbf{Z}$, we get the following identity in $K(B)$ :

$$
\begin{gathered}
\sum_{\alpha}(-1)^{\sum_{0<v}(v+1) \operatorname{dim} N_{v}} \operatorname{Ind}\left(D^{Y_{\alpha}} \otimes \mathcal{F}^{-p}(X) \otimes R_{i}(V),\right. \\
\left.m+\frac{1}{2} p^{2} e(N)+\frac{1}{2} p d^{\prime}(N), h\right) \\
=\sum_{\alpha}(-1)^{\sum_{0<v} \operatorname{dim} N_{v}} \operatorname{Ind}\left(D^{Y_{\alpha}} \otimes \mathcal{F}^{-p+1}(X) \otimes R_{i}(V)\right. \\
\left.m+\frac{1}{2}(p-1)^{2} e(N)+\frac{1}{2}(p-1) d^{\prime}(N), h\right) .
\end{gathered}
$$

As $\frac{1}{2} p(T X)_{S^{1}}$ is well defined in $H_{S^{1}}^{*}(M, \mathbf{Z})$, from (2.11), we know $\sum_{0<v} v \operatorname{dim} N_{v}=0 \bmod (2)$, thus we get Theorem 2.5 . 


\subsection{Proof of Theorem 2.4 .}

From Proposition 2.1, Theorems 2.5 and 2.6, for $1 \leq i \leq 4, h, p \in \mathbf{Z}, p>0$ and $m \in \frac{1}{2} \mathbf{Z}$, we get the following identity in $K(B)$ :

$$
\begin{aligned}
& \operatorname{Ind}\left(D^{X} \otimes \bigotimes_{n=1}^{\infty} \operatorname{Sym}_{q^{n}}(T X) \otimes R_{i}(V), m, h\right) \\
&=\operatorname{Ind}\left(D^{X} \otimes \bigotimes_{n=1}^{\infty} \operatorname{Sym}_{q^{n}}(T X) \otimes R_{i}(V), m^{\prime}, h+2 p e\right) .
\end{aligned}
$$

with

$$
m^{\prime}=m+p h+p^{2} e .
$$

Note that, from (2.1) and (2.13), if $m<0$, for $h \in \mathbf{Z}$, we have

$$
\operatorname{Ind}\left(D^{X} \otimes \bigotimes_{n=1}^{\infty} \operatorname{Sym}_{q^{n}}(T X) \otimes R_{i}(V), m, h\right)=0 \quad \text { in } \quad K(B) .
$$

i) Assume that $e=0$. Let $h \in \mathbf{Z}, m_{0} \in \frac{1}{2} \mathbf{Z}$ and $h \neq 0$ be fixed. If $h>0$, we take $m^{\prime}=m_{0}$, then for $p$ large enough, we get $m<0$ in (2.29). If $h<0$, we take $m=m_{0}$, then for $p$ large enough, we get $m^{\prime}<0$ in (2.29). From (2.30), we know that for $h \neq 0, m_{0} \in \frac{1}{2} \mathbf{Z}$ and $1 \leq i \leq 4$, we get

$$
\operatorname{Ind}\left(D^{X} \otimes \bigotimes_{n=1}^{\infty} \operatorname{Sym}_{q^{n}}(T X) \otimes R_{i}(V), m_{0}, h\right)=0 \quad \text { in } \quad K(B) .
$$

ii) Assume that $e<0$. For $h \in \mathbf{Z}, m_{0} \in \frac{1}{2} \mathbf{Z}, 1 \leq i \leq 4$, we take $m=m_{0}$, then for $p$ large enough, we get $m^{\prime}<0$ in (2.29). From (2.30), we again get (2.31). The proof of Theorem 2.4 is complete.

Remark 2.3. It might be suitable to add a remark on the comparison of the various proofs of the Witten rigidity theorem given in $[\mathbf{T}],[\mathbf{B T}],[\mathbf{L i u 1}]$ and the present paper. On one hand, the proofs in [BT] and [Liu1] rely on the Atiyah-Bott-Segal-Singer fixed point formula (cf. [ASe], [ASi]) and elliptic function theory, so they don't work on the $K$-theory level. This is reflected in [LM1] where a proof of a family Witten rigidity theorem on the equivariant Chern character level is given by extending the method in [Liu1]. On the other hand, consider the proofs given in $[\mathbf{T}],[\mathbf{B T}]$ and the present paper (for the last see Section 4 for more details). All these proofs rely on 
Taubes's idea that in certain steps one needs to consider Dirac operators on the fixed point set of the induced $\mathbf{Z}_{n}$-actions. This requires that the topoiogical conditions imposed in these proofs are for half of the equivariant first Pontryagin classes. However the proof in [Liu1] works directly on the fixed point set of the $S^{1}$-action, and thus works under weaker conditions on the equivariant first Pontryagin classes without the factor $\frac{1}{2}$. This leads to the natural question that whether a $K$-theory version of the proof in [Liu1] exists. Very likely one needs to use Hecke operators in the theory of modular forms to understand the shift operators and the modular transformations.

\subsection{Proof of Theorem 2.3 .}

In fact, by setting $V=0$ in (2.12), we know that

$$
\sum_{0<v} v^{2} \operatorname{dim} N_{v}=-2 e .
$$

Thus the case $e>0$ can never happen. If $e=0$, then all the numbers $\operatorname{dim} N_{v}$ are zero, so that the $S^{1}$-action can not have fixed points. From Theorem 1.1 , we know that the index bundle is zero in $K_{S^{1}}(B)$. For $e<0$, one may take $V=0$ in Theorem 2.4 to derive Theorem 2.3. The proof of Theorem 2.3 is complete.

\section{Proof of Theorem 2.6.}

In this section, we will prove Theorem 2.6 by introducing some shift operators as in $[\mathbf{T}, \S 7]$. This section is organized as follows: In Section 3.1, we introduce some notations. In Section 3.2, we prove Theorem 2.6 by introducing some shift operators as in $[\mathbf{T}, \S 7]$.

Throughout this section, we use the notations of Section 2.

\subsection{Reformulation of Theorem 2.6.}

To simplify the notations, we introduce some new notations in this subsection.

For $n_{0} \in \mathbf{N}^{*}$, we define a number operator $P$ on $K_{S^{1}}(M)\left[\left[q^{\frac{1}{n_{0}}}\right]\right]$ in the following way: if $R(q)=\bigoplus_{n \in \frac{1}{n_{0}}} \mathbf{z}_{n} R_{n} q^{n} \in K_{S^{1}}(M)\left[\left[q^{\frac{1}{n_{0}}}\right]\right]$, then $P$ acts on $R(q)$ by multiplication by $n$ on $R_{n}$. From now on, we simply write $\operatorname{Sym}_{q^{n}}(T X), \Lambda_{q^{n}}(V)$ as $\operatorname{Sym}\left(T X_{n}\right), \Lambda\left(V_{n}\right)$ respectively. In this way, $P$ acts on $T X_{n}$ and $V_{n}$ by multiplication by $n$, and the actions $P$ on 
$\operatorname{Sym}\left(T X_{n}\right), \Lambda\left(V_{n}\right)$ are naturally induced from its actions on $T X_{n}$ and $V_{n}$, etc. So the eigenspace of $P=n$ is given by the coefficient of $q^{n}$ of the corresponding element $R(q)$. For $R(q)=\bigoplus_{n \in \frac{1}{n_{0}}} \mathbf{z} R_{n} q^{n} \in K_{S^{1}}(M)\left[\left[q^{\frac{1}{n_{0}}}\right]\right]$, we will also write

$$
\text { Ind }\left(D^{X} \otimes R(q), m, h\right)=\operatorname{Ind}\left(D^{X} \otimes R_{m}, h\right) .
$$

Let $H$ be the canonical basis of $\operatorname{Lie}\left(S^{1}\right)=\mathbf{R}$, i.e., $\exp (t H)=\exp (2 \pi i t)$ for $t \in \mathbf{R}$. If $E$ is an $S^{1}$-equivariant vector bundle over $M$, let $L_{H}$ denote the corresponding infinitesimal action of $H$ on $\Gamma(M, E)$, the set of smooth sections of $E$ on $M$.

On the fixed point set $F$, let $J_{H}$ be the representation of $\operatorname{Lie}\left(S^{1}\right)$ on $\left.E\right|_{F}$. Then on $\Gamma\left(F,\left.E\right|_{F}\right), L_{H}$ is exactly the operator $J_{H}$ on $\Gamma\left(F,\left.E\right|_{F}\right)$, and the weight of the $S^{1}$ action on $\Gamma\left(F,\left.E\right|_{F}\right)$ is given by the action

$$
\mathbf{J}_{H}=\frac{-1}{2 \pi} \sqrt{-1} J_{H}
$$

Recall that the $\mathbf{Z}_{2}$ grading on $S(T X) \otimes \bigotimes_{n=1}^{\infty} \operatorname{Sym}\left(T X_{n}\right)$ (resp. $\left.S\left(T Y, \otimes_{0<v}\left(\operatorname{det} N_{v}\right)^{-1}\right) \otimes \mathcal{F}^{-p}(X)\right)$ is induced by the $\mathbf{Z}_{2}$-grading on $S(T X)$ (resp. $\left.S\left(T Y, \otimes_{0<v}\left(\operatorname{det} N_{v}\right)^{-1}\right)\right)$. Write

$$
F_{V}^{1}=S(V) \otimes \bigotimes_{n=1}^{\infty} \Lambda\left(V_{n}\right), \quad F_{V}^{2}=\bigotimes_{n \in \mathrm{N}+\frac{1}{2}} \Lambda\left(V_{n}\right) .
$$

There are two natural $\mathbf{Z}_{2}$ gradings on $F_{V}^{1}, F_{V}^{2}$. The first grading is induced by the $\mathbf{Z}_{2}$-grading of $S(V)$ and the nature $\mathbf{Z}_{2}$ grading (induced by forms of homogeneous degree ) of $\bigotimes_{n=1}^{\infty} \Lambda\left(V_{n}\right)$ and $\bigotimes_{n \in \mathbf{N}+\frac{1}{2}} \Lambda\left(V_{n}\right)$. We define $\tau_{e \mid F_{V}^{i \pm}}= \pm 1$ to be the involution defined by this $\mathbf{Z}_{2}$-grading. The second grading is the one for which $F_{V}^{i}(i=1,2)$ are purely even, i.e., $F_{V}^{i+}=F_{V}^{i}$. We denote by $\tau_{s}=$ Id the involution defined by this $\mathbf{Z}_{2}$ grading. Then the coefficient of $q^{n}\left(n \in \frac{1}{2} \mathbf{Z}\right)$ in (2.13) of $R_{1}(V), R_{2}(V)$ (resp. $R_{3}(V), R_{4}(V)$ ) is exactly the $\mathbf{Z}_{2}$-graded vector sub-bundle of $\left(F_{V}^{1}, \tau_{s}\right),\left(F_{V}^{1}, \tau_{e}\right)$ (resp. $\left(F_{V}^{2}, \tau_{e}\right)$, $\left.\left(F_{V}^{2}, \tau_{s}\right)\right)$, on which $P$ acts by multiplication by $n$.

We will denote by $\tau_{e}$ (resp. $\tau_{s}$ ) the $\mathbf{Z}_{2}$-grading on $S(T X) \otimes$ $\bigotimes_{n=1}^{\infty} \operatorname{Sym}\left(T X_{n}\right) \otimes F_{V}^{i}$ induced by the above $\mathbf{Z}_{2}$-gradings.

Let $h^{V_{v}}$ be the metric on $V_{v}$ induced by the metric $h^{V}$ on $V$. In the following, we will identify $\Lambda V_{v}$ with $\Lambda \bar{V}_{v}^{*}$ by using the Hermitian metric $h^{V_{v}}$. By (2.8), as in (1.12), there is a natural isomorphism between $\mathbf{Z}_{2}$-graded 
$C(V)$-Clifford modules over $F$,

$$
\left.S\left(V_{0}^{\mathbf{R}}, \otimes_{0<v}\left(\operatorname{det} V_{v}\right)^{-1}\right) \otimes \bigotimes_{0<v} \Lambda V_{v} \simeq S(V)\right|_{F} .
$$

By using above notations, on the fixed point set $F$, we rewrite (2.15), for $p \in \mathbf{N}$,

$$
\begin{aligned}
& \mathcal{F}_{p}(X)=\bigotimes_{0<v}\left(\bigotimes_{n=1}^{\infty} \operatorname{Sym}\left(N_{v, n}\right) \otimes \bigotimes_{\substack{n \in \mathbb{N} \\
n>p v}} \operatorname{Sym}\left(\bar{N}_{v, n}\right)\right) \otimes \bigotimes_{n=1}^{\infty} \operatorname{Sym}\left(T Y_{n}\right) \\
& \mathcal{F}_{p}^{\prime}(X)=\bigotimes_{\substack{0<v, n \in \mathbb{N}, 0 \leq n \leq p v}}\left(\operatorname{Sym}\left(N_{v,-n}\right) \otimes \operatorname{det} N_{v}\right) \\
& \mathcal{F}^{-p}(X)=\mathcal{F}_{p}(X) \otimes \mathcal{F}_{p}^{\prime}(X) .
\end{aligned}
$$

Let $V_{0}=V_{0}^{\mathbf{R}} \otimes_{\mathbf{R}}$ C. From (2.7), (3.3) and (3.4), we get

$$
\begin{aligned}
\mathcal{F}^{0}(X)= & \bigotimes_{n=1}^{\infty} \operatorname{Sym}\left(\bigoplus_{0<v}\left(N_{v, n} \oplus \bar{N}_{v, n}\right)\right) \otimes \bigotimes_{n=1}^{\infty} \operatorname{Sym}\left(T Y_{n}\right) \\
& \otimes \operatorname{Sym}\left(\bigoplus_{0<v} N_{v, 0}\right) \otimes \operatorname{det}\left(\bigoplus_{0<v} N_{v}\right) \\
F_{V}^{1}= & \bigotimes_{n=1}^{\infty} \Lambda\left(\bigoplus_{0<v}\left(V_{v, n} \oplus \bar{V}_{v, n}\right) \oplus V_{0, n}\right) \\
& \otimes S\left(V_{0}^{\mathbf{R}}, \otimes_{0<v}\left(\operatorname{det} V_{v}\right)^{-1}\right) \otimes \bigotimes_{0<v} \Lambda\left(V_{v, 0}\right) \\
F_{V}^{2}= & \bigotimes_{0<n \in \mathbf{Z}+1 / 2} \Lambda\left(\bigoplus_{0<v}\left(V_{v, n} \oplus \bar{V}_{v, n}\right) \oplus V_{0, n}\right) .
\end{aligned}
$$

Now, we can reformulate Theorem 2.6 as the following Theorem.

Theorem 3.1. For each $\alpha, h, p \in \mathbf{Z}, p>0, m \in \frac{1}{2} \mathbf{Z}$, for $i=1,2, \tau=\tau_{e}$ or $\tau_{s}$, we have the following identity in $K(B)$,

$$
\begin{aligned}
\operatorname{Ind}_{\tau}\left(D^{Y_{\alpha}} \otimes\right. & \left.\mathcal{F}^{-p}(X) \otimes F_{V}^{i}, m+\frac{1}{2} p^{2} e(N)+\frac{1}{2} p d^{\prime}(N), h\right) \\
& =\operatorname{Ind}_{\tau}\left(D^{Y_{\alpha}} \otimes \mathcal{F}^{0}(X) \otimes F_{V}^{i}, m+p h+p^{2} e, h+2 p e\right) .
\end{aligned}
$$

Proof: The rest of this section is devoted to the proof of Theorem 3.1. 


\subsection{Proof of Theorem 3.1.}

Inspired by [T, $\S 7]$, for $p \in \mathbf{N}^{*}$, we define the shift operators,

$$
\begin{aligned}
& r_{*}: N_{v, n} \rightarrow N_{v, n+p v}, \quad r_{*}: \bar{N}_{v, n} \rightarrow \bar{N}_{v, n-p v}, \\
& r_{*}: V_{v, n} \rightarrow V_{v, n+p v}, \quad r_{*}: \bar{V}_{v, n} \rightarrow \bar{V}_{v, n-p v} .
\end{aligned}
$$

This means that we change the action of the operator $P$ on $N_{v, n}$ (resp. $\bar{N}_{v, n}$ ) by $n+p v$ (resp. $n-p v$ ), etc.

Recall that $L(N), L(V)$ are the complex line bundles over $F$ defined by (2.10). Also recall that $L=L(N)^{-1} \otimes L(V)$ is a trivial complex line bundle on $F$, and $g \in S^{1}$ acts on it by multiplication by $g^{2 e}$.

Proposition 3.1. For $p \in \mathbf{Z}, p>0, i=1,2$, there are natural isomorphisms of vector bundles over $F$,

$$
\begin{aligned}
& r_{*}\left(\mathcal{F}^{-p}(X)\right) \simeq \mathcal{F}^{0}(X) \otimes L(N)^{p} \\
& r_{*}\left(F_{V}^{i}\right) \simeq F_{V}^{i} \otimes L(V)^{-p}
\end{aligned}
$$

Proof: 1) Under the action of the shift operator $r_{*}$,

$$
\begin{gathered}
r_{*}\left(\mathcal{F}_{p}^{\prime}(X)\right)=\bigotimes_{\substack{0<v \\
0 \leq n \leq p v}}\left(\operatorname{Sym}\left(N_{v,-n+p v}\right) \otimes \operatorname{det} N_{v}\right) \\
\quad=\bigotimes_{\substack{0<v \\
0 \leq n \leq p v}} \operatorname{Sym}\left(N_{v, n}\right) \otimes \bigotimes_{0<v} \operatorname{det} N_{v} \otimes L(N)^{p}
\end{gathered}
$$

From (3.5), (3.10), we get the first equation of (3.9).

2) For $F_{V}^{i}(i=1,2)$, we only need to consider the shift operator on the following elements

$$
\begin{aligned}
& F_{V, F}^{1}=\bigotimes_{n=1}^{\infty} \Lambda\left(\bigoplus_{0<v}\left(V_{v, n} \oplus \bar{V}_{v, n}\right)\right) \otimes \bigotimes_{0<v} \Lambda\left(V_{v, 0}\right), \\
& F_{V, F}^{2}=\bigotimes_{n \in \mathrm{N}+1 / 2} \Lambda\left(\bigoplus_{0<v}\left(V_{v, n} \oplus \bar{V}_{v, n}\right)\right) .
\end{aligned}
$$

We compute easily that

$$
\begin{aligned}
& r_{*} F_{V, F}^{1}=\bigotimes_{n=1}^{\infty} \Lambda\left(\bigoplus_{0<v}\left(V_{v, n+p v} \oplus \bar{V}_{v, n-p v}\right)\right) \otimes \bigotimes_{0<v} \Lambda\left(V_{v, p v}\right), \\
& r_{*} F_{V, F}^{2}=\bigotimes_{n \in \mathrm{N}+1 / 2} \Lambda\left(\bigoplus_{0<v}\left(V_{v, n+p v} \oplus \bar{V}_{v, n-p v}\right)\right) .
\end{aligned}
$$


The Hermitian metric $h^{V_{v}}$ on $V_{v}$ induces a natural isomorphism of complex vector bundles over $F$ :

$$
\Lambda^{i} \bar{V}_{v} \simeq \Lambda^{\operatorname{dim} V_{v}-i} V_{v} \otimes \operatorname{det} \bar{V}_{v} .
$$

In fact, let $d v_{V_{v}}$ be the volume form on $\left(V_{v, \mathbf{R}}, h^{V_{v}}\right)$, then we define $\Phi$ : $\Lambda^{\operatorname{dim} V_{v}-i} V_{v} \otimes \operatorname{det} \bar{V}_{v} \rightarrow\left(\Lambda^{i} V_{v}\right)^{*}$ as follows: for $s_{1} \in \Lambda^{\operatorname{dim} V_{v}-i} V_{v} \otimes \operatorname{det} \bar{V}_{v}, s_{2} \in$ $\Lambda^{i} V_{v}$

$$
\Phi\left(s_{1}\right)\left(s_{2}\right) d v_{V_{v}}=s_{1} \wedge s_{2} .
$$

Clearly, $\Phi$ is an isomorphism of complex vector bundles. By using the Hermitian metric $h^{V_{v}}$, we identify $\left(\Lambda^{i} V_{v}\right)^{*}$ to $\Lambda^{i} \bar{V}_{v}$.

For $n \in \mathbf{N}, 0<n \leq p v, 0 \leq i \leq \operatorname{dim} V_{v}$, (3.13) induces a natural $S^{1}$-equivariant isomorphism of complex vector bundles

$$
\begin{aligned}
& \Lambda^{i} \bar{V}_{v, n-p v} \simeq \Lambda^{\operatorname{dim} V_{v}-i} V_{v,-n+p v} \otimes \operatorname{det} \bar{V}_{v}, \\
& \Lambda^{i} \bar{V}_{v, n-p v-\frac{1}{2}} \simeq \Lambda^{\operatorname{dim} V_{v}-i} V_{v,-n+p v+\frac{1}{2}} \otimes \operatorname{det} \bar{V}_{v} .
\end{aligned}
$$

This means

$$
\begin{aligned}
& \bigotimes_{n \in \mathbf{N}, 0<n \leq p v} \Lambda^{i_{n}} \bar{V}_{v, n-p v} \simeq \bigotimes_{n \in \mathbf{N}, 0<n \leq p v}\left(\Lambda^{\operatorname{dim} V_{v}-i_{n}} V_{v,-n+p v} \otimes \operatorname{det} \bar{V}_{v}\right), \\
& \bigotimes_{n \in \mathbf{N}, 0 \leq n<p v} \Lambda^{i_{n}} \bar{V}_{v, n-p v+\frac{1}{2}} \simeq \bigotimes_{n \in \mathbf{N}, 0 \leq n<p v}\left(\Lambda^{\operatorname{dim} V_{v}-i_{n}} V_{v,-n+p v-\frac{1}{2}} \otimes \operatorname{det} \bar{V}_{v}\right) .
\end{aligned}
$$

From (2.10) and the isomorphisms (3.12) and (3.15) of complex vector bundles over $F$, one gets the second induced isomorphism in (3.9). The proof of Proposition 3.1 is complete.

Proposition 3.2. For $p \in \mathbf{Z}, p>0, i=1,2$, the bundle isomorphism induced by (3.9),

$$
\begin{aligned}
r_{*}: S\left(T Y, \otimes_{0<v}\left(\operatorname{det} N_{v}\right)^{-1}\right) \otimes \mathcal{F}^{-p}(X) \otimes F_{V}^{i} \\
\rightarrow S\left(T Y, \otimes_{0<v}\left(\operatorname{det} N_{v}\right)^{-1}\right) \otimes \mathcal{F}^{0}(X) \otimes F_{V}^{i} \otimes L^{-p}
\end{aligned}
$$

verifies the following identities:

$$
\begin{aligned}
& r_{*}^{-1} \cdot \mathbf{J}_{H} \cdot r_{*}=\mathbf{J}_{H}, \\
& r_{*}^{-1} \cdot P \cdot r_{*}=P+p \mathbf{J}_{H}+p^{2} e-\frac{1}{2} p^{2} e(N)-\frac{p}{2} d^{\prime}(N) .
\end{aligned}
$$

For the $\mathbf{Z}_{2}$-gradings, we have

$$
\tau_{e} r_{*}=r_{*} \tau_{e}, \quad \tau_{s} r_{*}=r_{*} \tau_{s} .
$$


Proof: The first equation of (3.17) is obvious.

To prove the second eciuation of (3.17) we divide the argument into several steps.

a) From (3.15), on $\bigotimes_{\substack{n \in \mathbb{N} \\ 0<n \leq p v}} \Lambda^{i_{n}} \bar{V}_{v, n}$, we have

$$
\begin{aligned}
& r_{*}^{-1} P r_{*}= \sum_{\substack{n \in \mathbb{N} \\
0<n \leq p v}}\left(\operatorname{dim} V_{v}-i_{n}\right)(-n+p v) \\
&=P+p \mathbf{J}_{H}+\sum_{\substack{n \in \mathbb{N} \\
0<n \leq p v}}(-n+p v) \operatorname{dim} V_{v} \\
& \quad=P+p \mathbf{J}_{H}+\frac{1}{2}\left(p^{2} v^{2}-p v\right) \operatorname{dim} V_{v} .
\end{aligned}
$$

Thus, from (2.9), (3.19), on $\bigotimes_{0<v} \bigotimes_{\substack{n \in \mathbb{N} \\ 0<n \leq p v}} \Lambda^{i_{n}} \bar{V}_{v, n}$, we have

$$
r_{*}^{-1} P r_{*}=F^{\prime}+p \mathbf{J}_{H}+\frac{1}{2} p^{2} e(V)-\frac{1}{2} p d^{\prime}(V) .
$$

The operators $P$ and $\mathbf{J}_{H}$ act on $S\left(V_{0}^{\mathbf{R}}, \operatorname{det}\left(\oplus_{0<v} V_{v}\right)^{-1}\right)$ by multiplication by

$$
0, \quad-\frac{1}{2} \sum_{0<v} v \operatorname{dim} V_{v}=-\frac{1}{2} d^{\prime}(V)
$$

respectively.

When $P$ acts the rest oart of $F_{V}^{1}$, we have

$$
r_{*}^{-1} P r_{*}=P+p \mathbf{J}_{H} \text {. }
$$

From (3.6), (3.20), (3.21) and (3.22), we know that when acting on $F_{V}^{1}$, one has the equality:

$$
r_{*}^{-1} \operatorname{Pr}_{*}=P+p \mathbf{J}_{H}+\frac{1}{2} p^{2} e(V) .
$$

b) Similar to (3.20), from (3.15), on $\bigotimes_{\substack{n \in \mathbb{N} \\ 0 \leq n<p v}} \Lambda^{i_{n}} \bar{V}_{v, n+\frac{1}{2}}$, we have

$$
\begin{aligned}
r_{*}^{-1} P r_{*}=\sum_{\substack{n \in \mathbb{N} \\
0 \leq n<p v}}\left(\operatorname{dim} V_{v}-i_{n}\right)\left(-n+p v-\frac{1}{2}\right) \\
=P+p \mathbf{J}_{H}+\left(\operatorname{dim} V_{v}\right) \sum_{\substack{n \in \mathbf{N} \\
0 \leq n<p v}}\left(-n+p v-\frac{1}{2}\right) \\
=P+p \mathbf{J}_{H}+\frac{1}{2} p^{2} v^{2} \operatorname{dim} V_{v} .
\end{aligned}
$$


From (2.9), (3.24), on $\bigotimes_{0<v} \bigotimes_{\substack{n \in \mathbb{N} \\ 0 \leq n<p v}} \Lambda^{i_{n}} \bar{V}_{v, n+\frac{1}{2}}$, we have

$$
r_{*}^{-1} P r_{*}=P+p \mathbf{J}_{H}+\frac{1}{2} p^{2} e(V) .
$$

When $P$ acts on the rest part of $F_{V}^{2}$, one has

$$
r_{*}^{-1} P r_{*}=P+p \mathbf{J}_{H} \text {. }
$$

From (3.25), (3.26), on $F_{V}^{2}$, we again have (3.23).

c) Note that on $\bigotimes_{0<v, 0 \leq n \leq p v} \operatorname{det} N_{v}, \mathbf{J}_{H}$ acts as $p e(N)+d^{\prime}(N)$. By (3.5), (3.10), we know that on $\mathcal{F}^{-p}(X)$,

$$
r_{*}^{-1} P r_{*}=P+p \mathbf{J}_{H}-p\left(p e(N)+d^{\prime}(N)\right) .
$$

On $S\left(T Y, \operatorname{det}\left(\oplus_{0<v} N_{v}\right)^{-1}\right), \mathbf{J}_{H}$ acts as $-\frac{1}{2} d^{\prime}(N)$. So on $S\left(T Y, \operatorname{det}\left(\oplus_{0<v} N_{v}\right)^{-1}\right)$ $\otimes \mathcal{F}^{-p}(X)$

$$
r_{*}^{-1} P r_{*}=P+p \mathbf{J}_{H}-p^{2} e(N)-\frac{1}{2} p d^{\prime}(N) .
$$

From (2.12), (3.23), b) and (3.28), we get the second equation of (3.17).

Finally, under our operations, the $\mathbf{Z}_{2}$-grading $\tau_{s}$ does not change. For the $\mathbf{Z}_{2}$-grading $\tau_{e}$, it changes only on $\bigotimes_{0<v} \bigotimes_{\substack{n \in \mathbb{N} \\ 0<n \leq p v}} \Lambda^{i_{n}} \bar{V}_{v, n}$ of $F_{V}^{1}$ (resp. on $\bigotimes_{0<v} \bigotimes_{\substack{n \in \mathbb{N} \\ 0 \leq n<p v}} \Lambda^{i_{n}} \bar{V}_{v, n+\frac{1}{2}}$ of $F_{V}^{2}$ ). From (3.15), we know that

$$
r_{*}^{-1} \tau_{e} r_{*}=(-1)^{\sum_{0<v} p v \operatorname{dim} V_{v}} \tau_{e} .
$$

As $\frac{1}{2} p_{1}(V)_{S^{1}} \in H_{S^{1}}^{*}(M, \mathbf{Z})$ is well-defined, from (2.5), (2.11), we get

$$
\sum_{0<v} v \operatorname{dim} V_{v}=0 \bmod (2),
$$

From (3.29), (3.30), we get (3.18). The proof of Proposition 3.2 is complete.

Proof of Theorem 3.1: From (2.12) and Propositions 3.2, for each $\alpha$, $h, p \in \mathbf{Z}, p>0, m \in \frac{1}{2} \mathbf{Z}$, and for $i=1,2, \tau=\tau_{e}$ or $\tau_{s}$, we have the following identity in $K(B)$,

$$
\begin{array}{r}
\operatorname{Ind}_{\tau}\left(D^{Y_{\alpha}} \otimes \mathcal{F}^{-p}(X) \otimes F_{V}^{i}, m+\frac{1}{2} p^{2} e(N)+\frac{1}{2} p d^{\prime}(N), h\right) \\
=\operatorname{Ind}_{\tau}\left(D^{Y_{\alpha}} \otimes \mathcal{F}^{0}(X) \otimes F_{V}^{i} \otimes L^{-p}, m+p h+p^{2} e, h\right) \\
\quad=\operatorname{Ind}_{\tau}\left(D^{Y_{\alpha}} \otimes \mathcal{F}^{0}(X) \otimes F_{V}^{i}, m+p h+p^{2} e, h+2 p e\right) .
\end{array}
$$

The proof of Theorem 3.1 is complete. 


\section{Proof of Theorem 2.7.}

In this section, we prove Theorem 2.7. Many arguments in this section are inspired by $[\mathbf{T}, \S 6,9]$. We will construct a family twisted Dirac operator on $M\left(n_{j}\right)$, the fixed point set of the induced $\mathbf{Z}_{n_{j}}$ action on $M$. By applying our $K$-theory version of the equivariant family index theorem to this operator, we derive Theorem 2.7 .

This section is organized as follows: In Section 4.1, we construct a family Dirac operator on $M\left(n_{j}\right)$. In Section 4.2 , by introducing a shift operator, we will relate both sides of equation (2.26) to the index bundle of the family Dirac operator on $M\left(n_{j}\right)$. In Section 4.3, we prove Theorem 2.7.

In this section, we make the same assumptions and use the same notations as in Sections 2 and 3.

\subsection{The $\mathrm{Spin}^{c}$ Dirac operator on $M\left(n_{j}\right)$.}

Let $\pi: M \rightarrow B$ be a fibration of compact manifolds with fiber $X$ and $\operatorname{dim}_{\mathrm{R}} X=2 l$. We assume that $S^{1}$ acts fiberwise on $M$, and $T X$ has an $S^{1}$-invariant spin structure. Let $V$ be a real vector bundle over $M$ carrying an $S^{1}$-invariant spin structure and $\operatorname{dim}_{\mathrm{R}} V=2 k$.

Let $F=\left\{F_{\alpha}\right\}$ be the fixed point set of the $S^{1}$ action on $M$. Then $\pi: F \rightarrow B$ is a fibration with compact fiber $Y$. For $n \in \mathbf{N}, n>0$, let $\mathbf{Z}_{n} \subset S^{1}$ denote the cyclic subgroup of order $n$.

For $n_{j} \in \mathbf{N}$ with $n_{j}>0$, let $M\left(n_{j}\right)$ be the fixed point set of the induced $\mathbf{Z}_{n_{j}}$-action on $M$. Then $\tau: M\left(n_{j}\right) \rightarrow B$ is a fibration with compact fiber $X\left(n_{j}\right)$. Let $N\left(n_{j}\right) \rightarrow M\left(n_{j}\right)$ be the normal bundle of $M\left(n_{j}\right)$ in $M$. Then we have the following $\mathbf{Z}_{n_{j}}$-equivariant decomposition of $N\left(n_{j}\right) \otimes_{\mathbf{R}} \mathbf{C}$ over $M\left(n_{j}\right)$,

$$
N\left(n_{j}\right) \otimes_{\mathbf{R}} \mathbf{C}=\bigoplus_{0<v<n_{j}} N\left(n_{j}\right)_{v} .
$$

Here $N\left(n_{j}\right)_{v}$ is the complex vector bundles over $M\left(n_{j}\right)$ with $g \in \mathbf{Z}_{n_{j}}$ acting by $g^{v}$ on it. Complex conjugation provides a $\mathbf{C}$ anti-linear isomorphism between $N\left(n_{j}\right)_{v}$ and $\overline{N\left(n_{j}\right)_{n_{j}-v}}$. If $n_{j}$ is even, this produces a real structure on $N\left(n_{j}\right)_{\frac{n_{j}}{2}}$, so this bundle is the complexification of a real vector bundle $N\left(n_{j}\right)_{\frac{n_{j}}{2}}^{\mathbf{R}}$ on $M\left(n_{j}\right)$. Thus, $N\left(n_{j}\right)$ is isomorphic, as a real vector bundle, to

$$
N\left(n_{j}\right) \simeq \bigoplus_{0<v<n_{j} / 2} N\left(n_{j}\right)_{v} \oplus N\left(n_{j}\right)_{\frac{n_{j}}{2}}^{\mathbf{R}} .
$$


Similarly, we have the following $\mathbf{Z}_{n_{j}}$-equivariant decomposition of $V \otimes_{\mathbf{R}}$ C,

$$
V \otimes_{\mathbf{R}} \mathbf{C}=\bigoplus_{0 \leq v<n_{j}} V\left(n_{j}\right)_{v}
$$

Here $V\left(n_{j}\right)_{v}$ is the complex vector bundle over $M\left(n_{j}\right)$ with $g \in \mathbf{Z}_{n_{j}}$ acting by $g^{v}$ on it. For $v \neq 0$, complex conjugation provides a $\mathbf{C}$ anti-linear isomorphism between $V\left(n_{j}\right)_{v}$ and $\overline{V\left(n_{j}\right)_{n_{j}-v}}$. If $n_{j}$ is even, this produces a real structure on $V\left(n_{j}\right)_{\frac{n_{j}}{2}}$, so this bundle is the complexification of a real vector bundle $V\left(n_{j}\right)_{\frac{n_{j}}{2}}^{\mathbf{R}}$ over $M\left(n_{j}\right)$. Complex conjugation also provides a real structure on $V\left(n_{j}\right)_{0}$ such that $V\left(n_{j}\right)_{0}=V\left(n_{j}\right)_{0}^{\mathbf{R}} \otimes_{\mathbf{R}} \mathbf{C}$. Thus, over $M\left(n_{j}\right)$, $V$ is isomorphic, as a real vector bundle, to

$$
\left.V\right|_{M\left(n_{j}\right)} \simeq V\left(n_{j}\right)_{0}^{\mathbf{R}} \oplus \bigoplus_{0<v<n_{j} / 2} V\left(n_{j}\right)_{v} \oplus V\left(n_{j}\right)_{\frac{n_{j}}{2}}^{\mathbf{R}} .
$$

In (4.2), (4.4), the last term is understood to be zero when $n_{j}$ is odd.

It is essential for us to know that the vector bundles $T X\left(n_{j}\right)$ and $V\left(n_{j}\right)_{0}^{\mathbf{R}}$ are orientable. For this we have the following Lemma which was proved in [E] (cf. [BT, Lemma 10.1]).

Lemma 4.1. Let $W$ be a real, spin vector bundle over a manifold $M$. We assume that $\mathbf{Z}_{n}\left(n \in \mathbf{N}^{*}\right)$ acts on $M$, and that the $\mathbf{Z}_{n}$ action lifts on $W$ and preserves the spin structure of $W$. Let $M(n)$ be the fixed point set of the $\mathbf{Z}_{n}$ action on $M$. Let $W_{0}$ be the subbundle of $W$ over $M(n)$ on which the generator of $\mathbf{Z}_{n}$ acts trivially. Then $W_{0}$ is orientable.

By Lemma 4.1, $T X\left(n_{j}\right)$ and $V\left(n_{j}\right)_{0}^{\mathbf{R}}$ are orientable over $M\left(n_{j}\right)$. Thus $N\left(n_{j}\right)$ is orientable over $M\left(n_{j}\right)$. By (4.2), (4.4), $N\left(n_{j}\right)_{\frac{n_{j}}{2}}^{\mathbf{R}}$ and $V\left(n_{j}\right)_{\frac{n_{j}}{2}}^{\mathbf{R}}$ are also orientable over $M\left(n_{j}\right)$. In the following, we fix the orientations of $N\left(n_{j}\right)_{\frac{n_{j}}{2}}^{\mathbf{R}}, V\left(n_{j}\right)_{\frac{n_{j}}{2}}^{\mathbf{R}}$ over $M\left(n_{j}\right)$. Then $T X\left(n_{j}\right)$ and $V\left(n_{j}\right)_{0}^{\mathbf{R}}$ are naturally oriented by $(4.2),(4.4)$ and the orientations of $T X, V, N\left(n_{j}\right)_{\frac{n_{j}}{2}}^{\mathbf{R}}, V\left(n_{j}\right)_{\frac{n_{j}}{2}}^{\mathbf{R}}$.

Let us denote by

$$
r\left(n_{j}\right)=\frac{1}{2}\left(1+(-1)^{n_{j}}\right) .
$$

Lemma 4.2. Assume that (2.6) holds. Let

$$
L\left(n_{j}\right)=\bigotimes_{0<v<n_{j} / 2}\left(\operatorname{det}\left(N\left(n_{j}\right)_{v}\right) \otimes \operatorname{det}\left(\overline{V\left(n_{j}\right)_{v}}\right)\right)^{\left(r\left(n_{j}\right)+1\right) v}
$$


be the line bundle over $M\left(n_{j}\right)$. Then:

i) $L\left(n_{j}\right)$ has an $n_{j}^{\text {th }}$ root over $M\left(n_{j}\right)$.

ii) Let

$$
\begin{aligned}
& L_{1}=\bigotimes_{0<v<n_{j} / 2}\left(\operatorname{det}\left(N\left(n_{j}\right)_{v}\right) \otimes \operatorname{det}\left(\overline{V\left(n_{j}\right)_{v}}\right)\right) \otimes L\left(n_{j}\right)^{r\left(n_{j}\right) / n_{j}} \\
& L_{2}=\bigotimes_{0<v<n_{j} / 2}\left(\operatorname{det}\left(N\left(n_{j}\right)_{v}\right)\right) \otimes L\left(n_{j}\right)^{r\left(n_{j}\right) / n_{j}}
\end{aligned}
$$

Let $U_{1}=T X\left(n_{j}\right) \oplus V\left(n_{j}\right)_{0}^{\mathbf{R}}$ and $U_{2}=T X\left(n_{j}\right) \oplus V\left(n_{j}\right)_{\frac{n_{j}}{2}}^{\mathbf{R}}$. Then $T X\left(n_{j}\right), V\left(n_{j}\right)_{\frac{n_{j}}{2}}^{\mathbf{R}}$ and $V\left(n_{j}\right)_{0}^{\mathbf{R}}$ are of even dimensions. Furthermore, $U_{1}$ (resp. $U_{2}$ ) has a Spinc structure defined by $L_{1}$ (resp. $L_{2}$ ).

Proof: By [BT, Lemma 9.4], TX $\left(n_{j}\right), V\left(n_{j}\right)_{\frac{n_{j}}{2}}^{\mathbf{R}}$ and $V\left(n_{j}\right)_{0}^{\mathbf{R}}$ are of even dimensions. From the procf of [BT, Lemmas 11.3 and 11.4], we get the rest part of Lemma 4.2.

Lemma 4.2 is very important. It allows us, as we are going to see, to apply the constructions and results in Section 1.3 to the fibration $M\left(n_{j}\right) \rightarrow B$, which is the main concern of this Section.

For $p_{j} \in \mathbf{N}, p_{j}<n_{j},\left(p_{j}, n_{j}\right)=1, \beta_{j}=\frac{p_{j}}{n_{j}}$, let us write

$$
\begin{gathered}
\mathcal{F}\left(\beta_{j}\right)=\bigotimes_{0<n \in \mathbf{Z}} \operatorname{Sym}\left(T X\left(r_{i j}\right)_{n}\right) \otimes \bigotimes_{0<v<n_{j} / 2} \operatorname{Sym}\left(\bigoplus_{0<n \in \mathbf{Z}+p_{j} v / n_{j}} N\left(n_{j}\right)_{v, n}\right. \\
\oplus \bigoplus_{V}^{1}\left(\beta_{j}\right)=\Lambda\left(\bigoplus_{0<n \in \mathbf{Z}-p_{j} v / n_{j}} \frac{}{N\left(n_{j}\right)_{v, n}}\right) \otimes \bigotimes_{0<n \in \mathbf{Z}+\frac{1}{2}} \operatorname{Sym}\left(N\left(n_{j}\right)_{\frac{n_{j}}{2}, n}\right), \\
\oplus\left(n_{j}\right)_{0, n} \oplus \bigoplus_{0<v<n_{j} / 2}\left(\bigoplus_{0<n \in \mathbf{Z}+p_{j} v / n_{j}} V\left(n_{j}\right)_{v, n}\right. \\
\left.\left.\bigoplus_{0<n \in \mathbf{Z}-p_{j} v / n_{j}} \overline{V\left(n_{j}\right)_{v, n}}\right) \oplus \bigoplus_{0<n \in \mathbf{Z}+\frac{1}{2}} V\left(n_{j}\right)_{\frac{n_{j}}{2}, n}\right)
\end{gathered}
$$




$$
\begin{array}{r}
F_{V}^{2}\left(\beta_{j}\right)=\Lambda\left(\bigoplus _ { 0 < n \in \mathbf { Z } } V ( n _ { j } ) _ { \frac { n _ { j } } { 2 } , n } \oplus \bigoplus _ { 0 < v < n _ { j } / 2 } \left(\bigoplus_{0<n \in \mathbf{Z}+p_{j} v / n_{j}+\frac{1}{2}} V\left(n_{j}\right)_{v, n}\right.\right. \\
\left.\left.\oplus \bigoplus_{0<n \in \mathbf{Z}-p_{j} v / n_{j}+\frac{1}{2}} \overline{V\left(n_{j}\right)_{v, n}}\right) \oplus \bigoplus_{0<n \in \mathbf{Z}+\frac{1}{2}} V\left(n_{j}\right)_{0, n}\right) .
\end{array}
$$

We denote by $D^{X\left(n_{j}\right)}$ the $S^{1}$-equivariant $\operatorname{Spin}^{c}$-Dirac operator on $S\left(U_{1}, L_{1}\right)$ or $S\left(U_{2}, L_{2}\right)$ along the fiber $X\left(n_{j}\right)$ defined as in Section 1.3. We denote by $D^{X\left(n_{j}\right)} \otimes \mathcal{F}\left(\beta_{j}\right) \otimes F_{V}^{i}\left(\beta_{j}\right)(i=1,2)$ the corresponding twisted $\operatorname{Spin}^{c}$ Dirac operator on $S\left(U_{i}, L_{i}\right) \otimes \mathcal{F}\left(\beta_{j}\right) \otimes F_{V}^{i}\left(\beta_{j}\right)$ along the fiber $X\left(n_{j}\right)$.

Remark 4.1. In fact, to define an $S^{1}$-action on $L\left(n_{j}\right)^{r\left(n_{j}\right) / n_{j}}$, one must replace the $S^{1}$-action by its $n_{j}$-fold action. Here by abusing notation, we still say an $S^{1}$-action without causing any confusion.

In the rest of this subsection, we will reinterpret all of the above objects when we restrict ourselves to $F$, the fixed point set of the $S^{1}$ action. We will use the notation of Sections 1.3, 2 .

Let $N_{F / M\left(n_{j}\right)}$ be the normal bundle of $F$ in $M\left(n_{j}\right)$. Then by $(2.7)$,

$$
\begin{aligned}
& N_{F / M\left(n_{j}\right)}=\bigoplus_{0<v: v \in n_{j} \mathbf{z}} N_{v} \\
& T X\left(n_{j}\right) \otimes_{\mathbf{R}} \mathbf{C}=\left(T Y \otimes_{\mathbf{R}} \mathbf{C}\right) \oplus \bigoplus_{0<v, v \in n_{j} \mathbf{z}}\left(N_{v} \oplus \bar{N}_{v}\right) .
\end{aligned}
$$

By (2.7) and (4.1), the restriction of $N\left(n_{j}\right)_{v}\left(1 \leq v \leq n_{j} / 2\right)$ to $F$ is given by

$$
N\left(n_{j}\right)_{v}=\bigoplus_{0<v^{\prime}: v^{\prime}=v \bmod \left(n_{j}\right)} N_{v^{\prime}} \oplus \bigoplus_{0<v^{\prime}: v^{\prime}=-v \bmod \left(n_{j}\right)} \bar{N}_{v^{\prime}}
$$

By (2.8) and (4.4), the restriction of $V\left(n_{j}\right)_{v}\left(1 \leq v \leq n_{j} / 2\right)$ to $F$ is given by

$$
V\left(n_{j}\right)_{v}=\bigoplus_{0<v^{\prime}: v^{\prime}=v \bmod \left(n_{j}\right)} V_{v^{\prime}} \oplus \bigoplus_{0<v^{\prime}: v^{\prime}=-v \bmod \left(n_{j}\right)} \bar{V}_{v^{\prime}}
$$

and for $v=0$,

$$
V\left(n_{j}\right)_{0}=\left(V_{0}^{\mathbf{R}} \otimes_{\mathbf{R}} \mathbf{C}\right) \oplus \bigoplus_{0<v, v=0 \bmod \left(n_{j}\right)}\left(V_{v} \oplus \bar{V}_{v}\right) .
$$


From (4.9)-(4.12), we have the following identifications of real vector bundles over $F$,

$$
\begin{aligned}
& N\left(n_{j}\right)_{\frac{n_{j}}{2}}^{\mathbf{R}_{2}}=\bigoplus_{0<v, v=\frac{n_{j}}{2} \bmod \left(n_{j}\right)} N_{v}, \\
& T X\left(n_{j}\right)=T Y \oplus \bigoplus_{0<v, v=0 \bmod \left(n_{j}\right)} N_{v}, \\
& V\left(n_{j}\right)_{c^{\mathbf{R}}}^{\mathbf{R}}=V_{0}^{\mathbf{R}} \oplus \bigoplus_{0<v, v=0 \bmod \left(n_{j}\right)} V_{v}, \\
& V\left(n_{j}\right)_{\frac{n_{j}}{2}}^{\mathbf{R}}=\bigoplus_{0<v, v=\frac{n_{j}}{2} \bmod \left(n_{j}\right)} V_{v} .
\end{aligned}
$$

We denote by $V_{0}=V_{0}^{\mathrm{R}} \otimes_{\mathbf{R}} \mathbf{C}$ the complexification of $V_{0}^{\mathbf{R}}$ over $F$. As $\left(p_{j}, n_{j}\right)=1$, we know that, for $v \in \mathbf{Z}, p_{j} v / n_{j} \in \mathbf{Z}$ iff $v / n_{j} \in \mathbf{Z}$. Also, $p_{j} v / n_{j} \in \mathbf{Z}+\frac{1}{2}$ iff $v / n_{j} \in \mathbf{Z}+\frac{1}{2}$. Remark if $v=-v^{\prime} \bmod \left(n_{j}\right)$, then $\left\{n, 0<n \in \mathbf{Z}+\frac{p_{j} v}{n_{j}}\right\}=\left\{r_{i}, 0<n \in \mathbf{Z}-\frac{p_{j} v^{\prime}}{n_{j}}\right\}$. From (4.9)-(4.12), we then get

$$
\begin{aligned}
& \mathcal{F}\left(\beta_{j}\right)=\bigotimes_{0<n \in \mathbf{Z}} \operatorname{Sym}\left(T Y_{n}\right) \otimes \bigotimes_{0<v, v=0, \frac{n_{j}}{2} \bmod \left(n_{j}\right)} \bigotimes_{0<n \in \mathbf{Z}+\frac{p_{j} v}{n_{j}}} \operatorname{Sym}\left(N_{v, n} \oplus \bar{N}_{v, n}\right) \\
& \otimes \bigotimes_{0<v^{\prime}<n_{j} / 2} \operatorname{Sym}\left(\bigoplus_{v=v^{\prime}}\left(\bigoplus_{\bmod \left(n_{j}\right)} N_{0<n \in \mathbf{Z}+\frac{p_{j} v}{n_{j}}} N_{v, n} \oplus \bigoplus_{0<n \in \mathbf{Z}-\frac{p_{j} v}{n_{j}}} \bar{N}_{v, n}\right)\right. \\
& \left.\oplus \bigoplus_{v=-v^{\prime} \bmod \left(n_{j}\right)}\left(\underset{0<n \in \mathbf{Z}+\frac{p_{j} v}{n_{j}}}{\bigoplus} N_{v, n} \oplus \underset{0<n \in \mathbf{Z}-\frac{p_{j} v}{n_{j}}}{\bigoplus_{v, n}}\right)\right) \\
& F_{V}^{1}\left(\beta_{j}\right)=\Lambda\left[\bigoplus_{0<n \in \mathbf{Z}} V_{0, n} \oplus \bigoplus_{0<{ }^{\prime \prime}, v=0, \frac{n_{j}}{2} \bmod \left(n_{j}\right)}\left(\bigoplus_{0<n \in \mathbf{Z}+\frac{p_{j} v}{n_{j}}} V_{v, n} \oplus \underset{0<n \in \mathbf{Z}-\frac{p_{j} v}{n_{j}}}{\bigoplus} \bar{V}_{v, n}\right)\right. \\
& \left.\oplus \bigoplus_{0<v^{\prime}<n_{j} / 2}\left(\bigoplus_{v=v^{\prime},-v^{\prime} \bmod \left(n_{j}\right)}\left(\bigoplus_{0<n \in \mathbf{Z}+\frac{p_{j} v}{n_{j}}} V_{v, n} \oplus \underset{0<n \in \mathbf{Z}-\frac{p_{j} v}{n_{j}}}{\bigoplus_{v, n}}\right)\right)\right]
\end{aligned}
$$


$F_{V}^{2}\left(\beta_{j}\right)=\Lambda\left[\bigoplus_{0<n \in \mathbf{Z}+\frac{1}{2}} V_{0, n} \oplus \bigoplus_{0<v, v=0, \frac{n_{j}}{2} \bmod \left(n_{j}\right)}\left(\bigoplus_{0<n \in \mathbf{Z}+\frac{p_{j} v}{n_{j}}+\frac{1}{2}} V_{v, n} \oplus \bigoplus_{0<n \in \mathbf{Z}-\frac{p_{j} v}{n_{j}}+\frac{1}{2}} \bar{V}_{v, n}\right)\right.$

$\left.\oplus \bigoplus_{0<v^{\prime}<n_{j} / 2}\left(\bigoplus_{v=v^{\prime},-v^{\prime} \bmod \left(n_{j}\right)}\left(\bigoplus_{0<n \in \mathbf{Z}+\frac{p_{j} n^{v}}{n_{j}}+\frac{1}{2}} V_{v, n} \oplus \bigoplus_{0<n \in \mathbf{Z}-\frac{p_{j} v}{n_{j}}+\frac{1}{2}} \bar{V}_{v, n}\right)\right)\right]$.

Now we want to compare the spinor bundles over $F$. From (4.6), (4.7), (4.10) and (4.11), we find that over $F$ we have

$$
\begin{gathered}
L\left(n_{j}\right)^{\frac{r\left(n_{j}\right)}{n_{j}}}=\bigotimes_{0<v^{\prime}<n_{j} / 2}\left(\bigotimes_{v=v^{\prime} \bmod \left(n_{j}\right)}\left(\operatorname{det} N_{v} \otimes \operatorname{det} \bar{V}_{v}\right)^{2 v^{\prime}}\right. \\
\left.\bigotimes_{1} \bigotimes_{0<v^{\prime}<n_{j} / 2}\left(\operatorname{det} N_{v} \otimes \operatorname{det} \bar{V}_{v}\right)^{-2 v^{\prime}}\right)^{r\left(n_{j}\right) / n_{j}}, \\
\bigotimes_{v=v^{\prime} \bmod \left(n_{j}\right)}^{\otimes}\left(\operatorname{det} N_{v} \otimes \operatorname{det} \bar{V}_{v}\right) \\
\left.\bigotimes_{2}=\bigotimes_{0<v^{\prime}<n_{j} / 2}\left(\operatorname{det} N_{v} \otimes \operatorname{det} \bar{V}_{v}\right)^{-1}\right) \otimes L\left(n_{j}\right)^{r\left(n_{j}\right) / n_{j}}, \\
\left.\bigotimes_{v=-v^{\prime} \bmod \left(n_{j}\right)}^{\operatorname{det}\left(n_{j}\right)} N_{v} \otimes \bigotimes_{v=-v^{\prime} \bmod \left(n_{j}\right)}\left(\operatorname{det} N_{v}\right)^{-1}\right) \otimes L\left(n_{j}\right)^{r\left(n_{j}\right) / n_{j}} .
\end{gathered}
$$

From (4.13), over $F$, we have

$$
\begin{aligned}
& T X\left(n_{j}\right) \oplus V\left(n_{j}\right)_{0}^{\mathbf{R}}=T Y \oplus V_{0}^{\mathbf{R}} \oplus \bigoplus_{0<v, v=0 \bmod \left(n_{j}\right)}\left(N_{v} \oplus V_{v}\right), \\
& T X\left(n_{j}\right) \oplus V\left(n_{j}\right)_{\frac{n_{j}}{2}}^{\mathbf{R}}=T Y \oplus \bigoplus_{0<v, v=0 \bmod \left(n_{j}\right)} N_{v} \oplus \bigoplus_{0<v, v=\frac{n_{j}}{2} \bmod \left(n_{j}\right)} V_{v} .
\end{aligned}
$$

Recall that the $\operatorname{Spin}^{c}$ vector bundles $U_{1}$ and $U_{2}$ have been defined in Lemma 
4.2. Let us write

$$
\begin{gathered}
S\left(U_{1}, L_{1}\right)^{\prime}=S\left(T Y \oplus V_{0}^{\mathrm{R}}, L_{1} \otimes \bigotimes_{\substack{0<v, v=0 \bmod \left(n_{j}\right)}}\left(\operatorname{det} N_{v} \otimes \operatorname{det} V_{v}\right)^{-1}\right) \otimes \bigotimes_{\substack{0<v, v=0 \bmod \left(n_{j}\right)}} \Lambda V_{v}, \\
S\left(U_{2}, L_{2}\right)^{\prime}=S(T Y, L_{2} \otimes \bigotimes_{\substack{0<v, v=0 \bmod \left(n_{j}\right)}}\left(\operatorname{det} N_{v}\right)^{-1} \otimes \underbrace{}_{\substack{0<v, v=\frac{n_{j}}{2} \bmod \left(n_{j}\right)}}\left(\operatorname{det} V_{v}\right)^{-1})
\end{gathered}
$$$$
\otimes \bigotimes_{\substack{0<v, v=\frac{n_{j}}{2} \bmod \left(n_{j}\right)}} \Lambda V_{v} .
$$

Then from (1.47), (1.49) and (4.17), for $i=1,2$, we have the following isomorphism of Clifford modules over $F$,

$$
S\left(U_{i}, L_{i}\right) \simeq S\left(I_{i}, L_{i}\right)^{\prime} \otimes \Lambda\left(\bigoplus_{0<v, v=0 \bmod \left(n_{j}\right)} N_{v}\right)
$$

We define the $\mathbf{Z}_{2}$ gradings on $S\left(U_{i}, L_{i}\right)^{\prime}(i=1,2)$ as that induced by the $\mathbf{Z}_{2}$-gradings on $S\left(U_{i}, L_{i}\right)(i=1,2)$ and on $\Lambda\left(\bigoplus_{0<v, v=0 \bmod \left(n_{j}\right)} N_{v}\right)$ such that the isomorphism (4.18) preserves these $\mathbf{Z}_{2}$-gradings.

We define formally the following complex line bundles over $F$,

$$
\begin{aligned}
& L_{1}^{\prime}=\left[L_{1}^{-1} \otimes \bigotimes_{\substack{0<v, v=0 \bmod \left(n_{j}\right)}}\left(\operatorname{det} N_{v} \otimes \operatorname{det} V_{v}\right) \otimes \bigotimes_{0<v}\left(\operatorname{det} N_{v} \otimes \operatorname{det} V_{v}\right)^{-1}\right]^{1 / 2}, \\
& L_{2}^{\prime}=\left[L_{2}^{-1} \otimes \bigotimes_{\substack{0<v, v=0 \bmod \left(n_{j}\right)}} \operatorname{det} N_{v} \otimes \bigotimes_{\substack{0<v, v=n_{j} / 2 \bmod \left(n_{j}\right)}} \operatorname{det} V_{v} \otimes \bigotimes_{0<v}\left(\operatorname{det} N_{v}\right)^{-1}\right]^{1 / 2} .
\end{aligned}
$$

From Lemma 4.2, $w_{2}\left(U_{i}\right)=c_{1}\left(L_{i}\right) \bmod (2)$, by (1.11), (1.47), (4.13) and the assumption that $V$ is spin, one get $w_{2}\left(U_{i}\right)=c_{1}\left(L_{i}^{\prime 2} \otimes L_{i}\right) \bmod (2)$. Thus $c_{1}\left(L_{i}^{\prime 2}\right)=0 \bmod (2)$ for $i=1,2$. Thus $L_{1}^{\prime}, L_{2}^{\prime}$ are well defined complex line bundles over $F$. For later use, we also write down the following expressions 
of $L_{i}^{\prime}(i=1,2)$ which can be deduced from (4.15):

$$
\begin{gathered}
L_{1}^{\prime}=\left[L\left(n_{j}\right)^{-1 / n_{j}} \otimes \bigotimes_{0<v, v=\frac{n_{j}}{2} \bmod \left(n_{j}\right)}\left(\operatorname{det} N_{v} \otimes \operatorname{det} \bar{V}_{v}\right)\right]^{\frac{r\left(n_{j}\right)}{2}}\left(\operatorname{det} N_{v}\right)^{-1} \otimes \bigotimes_{0<v, \frac{n_{j}}{2}<v<n_{j} \bmod \left(n_{j}\right)}\left(\operatorname{det} V_{v}\right)^{-1}, \\
\otimes \bigotimes_{0<v, 0<v \leq \frac{n_{j}}{2} \bmod \left(n_{j}\right)} \bigotimes_{0}^{\prime}=\left[L\left(n_{j}\right)^{-1 / n_{j}} \otimes \bigotimes_{0<v, v=\frac{n_{j}}{2} \bmod \left(n_{j}\right)}\left(\operatorname{det} N_{v} \otimes \operatorname{det} V_{v}\right)\right]^{\frac{r\left(n_{j}\right)}{2}} \\
\otimes \bigotimes_{0<v, 0<v \leq \frac{n_{j}}{2} \bmod \left(n_{j}\right)}\left(\operatorname{det} N_{v}\right)^{-1} .
\end{gathered}
$$

From (4.15), (4.17) and the definition of $L_{i}^{\prime}(i=1,2)$, we get the following identifications of Clifford modules over $F$,

$$
\begin{gathered}
S\left(U_{1}, L_{1}\right)^{\prime} \otimes L_{1}^{\prime}=S\left(T Y, \otimes_{0<v}\left(\operatorname{det} N_{v}\right)^{-1}\right) \otimes S\left(V_{0}^{\mathbf{R}}, \otimes_{0<v}\left(\operatorname{det} V_{v}\right)^{-1}\right) \\
\otimes \Lambda\left(\bigoplus_{0<v, v=0 \bmod \left(n_{j}\right)} V_{v}\right) \\
S\left(U_{2}, L_{2}\right)^{\prime} \otimes L_{2}^{\prime}=S\left(T Y, \otimes_{0<v}\left(\operatorname{det} N_{v}\right)^{-1}\right) \otimes \Lambda\left(\bigoplus_{0<v, v=\frac{n_{j}}{2} \bmod \left(n_{j}\right)} V_{v}\right) .
\end{gathered}
$$

To compare the $\mathbf{Z}_{2}$-gradings in (4.20), we will compare explicitly the orientations. Recall that, if $\left(W, h^{W}\right)$ is a real Euclidean vector space of dimension $2 m$, and $J$ is a complex structure on $W$ which preserves $h^{W}$. Let $\left\{e_{i}, J e_{i}\right\}_{i=1}^{m}$ be an orthonormal basis of $\left(W, h^{W}\right)$. Then $W$ is canonically oriented, and its orientation is defined by the canonical Riemannian volume form

$$
\left(e_{1} \wedge J e_{1}\right) \wedge \cdots\left(e_{m} \wedge J e_{m}\right)=d v_{W} .
$$

Let $d v_{T X}, d v_{V}$ be the corresponding Riemannian volume forms on $\left(T X, h^{T X}\right)$ and $\left(V, h^{V}\right)$ which define the orientations of $T X, V$ over $M$. Let $d v_{N_{v}}$ and $d v_{\bar{N}_{v}}$ ( resp. $\left.d v_{V_{v}}, d v_{\bar{V}_{v}}\right)(0<v)$ be the canonical Riemannian volume forms on $N_{v, \mathbf{R}}$ and $\bar{N}_{v, \mathbf{R}}=N_{v, \mathbf{R}}$ (resp. $V_{v, \mathbf{R}}, \bar{V}_{v, \mathbf{R}}=V_{v, \mathbf{R}}$ ). Then through the identifications (1.12) and (3.4), the orientations of $T Y$ and $V_{0}^{\mathbf{R}}$ 
over $F$ are defined by the volume forms $d v_{T Y}$ and $d v_{V_{0}^{\mathbf{R}}}$ respectively such that

$$
d v_{T X}=d v_{T Y} \otimes \underset{0<v}{\bigotimes} d v_{N_{v}}, \quad d v_{V}=d v_{V_{0}^{\mathbf{R}}} \otimes \bigotimes_{0<v} d v_{V_{v}}
$$

By (4.2), (4.4), (4.10) and (4.11), the orientations of $T X\left(n_{j}\right)$ and $V\left(n_{j}\right)_{0}^{\mathbf{R}}$, when restricted to $F$, are given by

$$
\begin{aligned}
& \left.d v_{T X}\right|_{F}=d v_{T X\left(n_{j}\right)} \otimes \bigotimes_{0<v^{\prime}<n_{j} / 2}\left(\bigotimes_{v=v^{\prime} \bmod \left(n_{j}\right)} d v_{N_{v}} \otimes \bigotimes_{v=-v^{\prime} \bmod \left(n_{j}\right)} d v_{\bar{N}_{v}}\right) \otimes d v_{N\left(n_{j}\right)_{\frac{n_{j}}{2}}^{\mathbf{R}}}, \\
& \left.d v_{V}\right|_{F}=d v_{V\left(n_{j}\right)_{0}^{\mathbf{R}}} \otimes \bigotimes_{0<v^{\prime}<n_{j} / 2}(\bigotimes_{v=v^{\prime} \bmod \left(n_{j}\right)} d v_{V_{v}} \otimes \underbrace{}_{v=-v^{\prime} \bmod \left(n_{j}\right)} d v_{\bar{V}_{v}}) \otimes d v_{V\left(n_{j}\right)_{\frac{n_{j}}{2}}^{\mathbf{R}_{j}}}
\end{aligned}
$$

respectively.

Clearly, we have

$$
d v_{N_{v}}=(-1)^{\operatorname{dim} \cdot V_{v}} d v_{\bar{N}_{v}}, \quad d v_{V_{v}}=(-1)^{\operatorname{dim} V_{v}} d v_{\bar{V}_{v}} .
$$

From (4.21), (4.22) and (4.23), we get

$$
\begin{aligned}
& d v_{T X\left(n_{j}\right)}=(-1)^{\Delta\left(n_{j}, N\right)} d v_{T Y} \otimes \\
& d v_{V\left(n_{j}\right)_{0}^{\mathbf{R}}}=(-1)^{\Delta\left(n_{j}, V\right)} d v_{V_{0}^{\mathbf{R}}} \otimes \bigotimes_{0<v, v=0 \bmod \left(n_{j}\right)} d v_{N_{v}}, \\
& \bigotimes_{0<v, v=0 \bmod \left(n_{j}\right)} d v_{V_{v}},
\end{aligned}
$$

where

$$
\begin{aligned}
& \Delta\left(n_{j}, N\right)=\sum_{\frac{n_{j}}{2}<v^{\prime}<n_{j}} \sum_{0<v=v^{\prime} \bmod \left(n_{j}\right)} \operatorname{dim} N_{v}+o\left(N\left(n_{j}\right)_{\frac{n_{j}}{2}}^{\mathbf{R}}\right), \\
& \Delta\left(n_{j}, V\right)=\sum_{\frac{n_{j}}{2}<v^{\prime}<n_{j}} \sum_{0<v=v^{\prime} \bmod \left(n_{j}\right)} \operatorname{dim} V_{v}+o\left(V\left(n_{j}\right)_{\frac{n_{j}}{2}}^{\mathbf{R}_{j}}\right),
\end{aligned}
$$

with $o\left(N\left(n_{j}\right)_{\frac{n_{j}}{2}}^{\mathbf{R}}\right)=0$ or 1 (resp. $o\left(V\left(n_{j}\right)_{\frac{n_{j}}{2}}^{\mathbf{R}}\right)=0$ or 1 ), the value depends on whether the given orientation on $N\left(n_{j}\right)_{\frac{n_{j}}{2}}^{\mathbf{R}}\left(\right.$ resp. $\left.V\left(n_{j}\right)_{\frac{n_{j}}{2}}^{\mathbf{R}}\right)$ agrees or disagrees with the complex orientation of $\bigoplus_{v=\frac{n_{j}}{2} \bmod \left(n_{j}\right)} N_{v}$ (resp. $\left.\bigoplus_{v=\frac{n_{j}}{2} \bmod \left(n_{j}\right)} V_{v}\right)$. 
From (4.13), (4.18), (4.23) and (4.24), we see that, for the $\mathbf{Z}_{2}$-gradings induced by $\tau_{s}$, the difference of the $\mathbf{Z}_{2}$-gradings of $(4.20)$ is $(-1)^{\Delta\left(n_{j}, N\right)}$; for the $\mathbf{Z}_{2}$-gradings induced by $\tau_{e}$, the difference of the $\mathbf{Z}_{2}$-gradings of the first (resp. second) equation of $(4.20)$ is $(-1)^{\Delta\left(n_{j}, N\right)+\Delta\left(n_{j}, V\right)}$ (resp. $\left.(-1)^{\Delta\left(n_{j}, N\right)+o\left(V\left(n_{j}\right) \frac{\mathbf{R}}{n_{j}}\right.} \frac{}{2}\right)$.

\subsection{The Shift operators.}

Let $p \in \mathbf{N}^{*}$ be fixed. For any $1 \leq j \leq J_{0}$, inspired by [ $\left.\mathbf{T}, \S 9\right]$, we define the following shift operators $r_{j *}$ :

$$
\begin{aligned}
& r_{j *}: N_{v, n} \rightarrow N_{v, n+(p-1) v+p_{j} v / n_{j}}, \quad r_{j *}: \bar{N}_{v, n} \rightarrow \bar{N}_{v, n-(p-1) v-p_{j} v / n_{j}} \\
& r_{j *}: V_{v, n} \rightarrow V_{v, n+(p-1) v+p_{j} v / n_{j}}, \quad r_{j *}: \bar{V}_{v, n} \rightarrow \bar{V}_{v, n-(p-1) v-p_{j} v / n_{j}}
\end{aligned}
$$

This means that we change the action of the operator $P$ on $N_{v, n}$ by a multiplication by $n+(p-1) v+p_{j} v / n_{j}$, etc. If $E$ is a combination of the above bundles, we denote by $r_{j *} E$ the bundle on which the action of $P$ is changed in the above way.

Recall that the vector bundles $F_{V}^{i}(i=1,2)$ have been defined in (3.3), (3.6). From (2.22), (3.5), we get

$$
\begin{aligned}
\mathcal{F}_{p, j}(X) & =\mathcal{F}_{p}(X) \otimes \mathcal{F}_{p-1}^{\prime}(X) \\
& \otimes \bigotimes_{(v, n) \in \cup_{i=1}^{j} I_{i}^{p}}\left(\operatorname{Sym}\left(N_{v,-n}\right) \otimes \operatorname{det} N_{v}\right) \otimes \bigotimes_{(v, n) \in \bar{I}_{j}^{p}} \operatorname{Sym}\left(\bar{N}_{v, n}\right)
\end{aligned}
$$

Proposition 4.1. There are natural isomorphisms of vector bundles over $F$ :

$$
\begin{aligned}
r_{j *} \mathcal{F}_{p, j-1}(X) & \simeq \mathcal{F}\left(\beta_{j}\right) \otimes \bigotimes_{0<v, v=0 \bmod \left(n_{j}\right)} \operatorname{Sym}\left(\bar{N}_{v, 0}\right) \\
& \otimes \bigotimes_{0<v}\left(\operatorname{det} N_{v}\right)^{\left[\frac{p_{j} v}{n_{j}}\right]+(p-1) v+1} \otimes \bigotimes_{0<v, v=0 \bmod \left(n_{j}\right)}\left(\operatorname{det} N_{v}\right)^{-1}
\end{aligned}
$$




$$
\begin{gathered}
r_{j *} \mathcal{F}_{p, j}(X) \simeq \mathcal{F}\left(\beta_{j}\right) \otimes \bigotimes_{0<v, v=0 \bmod \left(n_{j}\right)} \operatorname{Sym}\left(N_{v, 0}\right) \otimes \bigotimes_{0<v}\left(\operatorname{det} N_{v}\right)^{\left[\frac{p_{j} v}{n_{j}}\right]+(p-1) v+1}, \\
r_{j *} F_{V}^{1} \simeq S\left(V_{0}^{\mathbf{R}}, \otimes_{0<v}\left(\operatorname{det} V_{v}\right)^{-1}\right) \otimes F_{V}^{1}\left(\beta_{j}\right) \otimes \bigotimes_{0<v, v=0 \bmod \left(n_{j}\right)} \Lambda\left(V_{v, 0}\right) \\
\otimes \bigotimes_{0<v}\left(\operatorname{det} \bar{V}_{v}\right)^{\left[\frac{p_{j} v}{n_{j}}\right]+(p-1) v}, \\
r_{j *} F_{V}^{2} \simeq F_{V}^{2}\left(\beta_{j}\right) \otimes \underset{\substack{\bigotimes_{n} \\
0<v, v=\frac{n}{i}^{-} \bmod \left(n_{j}\right)}}{\Lambda}\left(V_{v, 0}\right) \otimes \bigotimes_{0<v}\left(\operatorname{det} \bar{V}_{v}\right)^{\left[\frac{p_{j} v}{n_{j}}+\frac{1}{2}\right]+(p-1) v} .
\end{gathered}
$$

Proof: The proof is similar to that of Proposition 3.1. We divide it into several steps.

1) From (3.5), (4.26), we get

$$
r_{j *} \mathcal{F}_{p-1}^{\prime}(X)=\bigotimes_{\substack{0<v, n \in \mathbf{N} \\ 0 \leq n \leq(p-1) v}} \operatorname{Sym}\left(N_{v,-n+(p-1) v+\frac{p_{j} v}{n_{j}}}\right) \otimes \bigotimes_{0<v}\left(\operatorname{det} N_{v}\right)^{(p-1) v+1}
$$

Note that by (2.20), for $v \in J=\left\{v \in \mathbf{N} \mid\right.$ There exists $\alpha$ such that $N_{v} \neq 0$ on $\left.F_{\alpha}\right\}$, there are no integer in $] \frac{p_{j-1} v}{n_{j-1}}, \frac{p_{j} v}{n_{j}}\left[\right.$. So for $v \in J$ and $i_{0}=j-1, j$, the elements $(v, n) \in \cup_{i=1}^{i_{0}} l_{i}^{p}$ are $(v,(p-1) v+1), \cdots,\left(v,(p-1) v+\left[\frac{p_{i_{0}} v}{n_{i_{0}}}\right]\right)$. Furthermore,

$$
\begin{aligned}
& {\left[\frac{p_{j-1} v}{n_{j-1}}\right]=\left[\frac{p_{j} v}{n_{j}}\right]-1 \quad \text { if } \quad v=0 \quad \bmod \left(n_{j}\right)} \\
& {\left[\frac{p_{j-1} v}{n_{j-1}}\right]=\left[\frac{p_{j} v}{n_{j}}\right] \quad \text { if } \quad v \neq 0 \quad \bmod \left(n_{j}\right)}
\end{aligned}
$$


From (2.21), (4.26) and (4.30), we have

$$
\begin{aligned}
& r_{j *}\left(\bigotimes_{(v, n) \in \cup_{i=1}^{j-1} I_{i}^{p}}\left(\operatorname{Sym}\left(N_{v,-n}\right) \otimes \operatorname{det} N_{v}\right)\right) \\
& =\bigotimes_{(v, n) \in \cup_{i=1}^{j-1} I_{i}^{p}} \operatorname{Sym}\left(N_{v,-n+(p-1) v+\frac{p_{j} v}{n_{j}}}\right) \otimes \bigotimes_{0<v}\left(\operatorname{det} N_{v}\right)^{\left[\frac{p_{j} v}{n_{j}}\right]} \otimes \bigotimes_{\substack{0<v, \\
\text { mod }\left(n_{j}\right)}}\left(\operatorname{det} N_{v}\right)^{-1}, \\
& r_{j *}\left(\bigotimes_{(v, n) \in \cup_{i=1}^{j} I_{i}^{p}}\left(\operatorname{Sym}\left(N_{v,-n}\right) \otimes \operatorname{det} N_{v}\right)\right) \\
& =\bigotimes_{(v, n) \in \cup_{i=1}^{j} I_{i}^{p}} \operatorname{Sym}\left(N_{v,-n+(p-1) v+\frac{p_{j} v}{n_{j}}}\right) \otimes \bigotimes_{0<v}\left(\operatorname{det} N_{v}\right)^{\left[\frac{p_{j} v}{n_{j}}\right]} .
\end{aligned}
$$

From (3.5), (4.14), (4.27), (4.29), (4.31), one easily gets the first two equations in (4.28).

2) For $0<n \leq(p-1) v+\frac{p_{j} v}{n_{j}}, n \in \mathbf{Z}, 0 \leq i \leq \operatorname{dim} V_{v}$, (3.13) induces a natural $S^{1}$-equivariant isomorphism of complex vector bundles over $F$,

$$
\Lambda^{i} \bar{V}_{v, n-(p-1) v-\frac{p_{j} v}{n_{j}}} \simeq \Lambda^{\operatorname{dim} V_{v}-i} V_{v,-n+(p-1) v+\frac{p_{j} v}{n_{j}}} \otimes \operatorname{det} \bar{V}_{v}
$$

From (3.6) and (4.32), as in (3.9), we obtain the third equality of (4.28).

3) For $0<n \leq(p-1) v+\frac{p_{j} v}{n_{j}}+\frac{1}{2}, n \in \mathbf{Z}, 0 \leq i \leq \operatorname{dim} V_{v}$, (3.13) induces a natural $S^{1}$-equivariant isomorphism of complex vector bundles over $F$,

$$
\Lambda^{i} \bar{V}_{v, n-(p-1) v-\frac{p_{j} v}{n_{j}}-\frac{1}{2}} \simeq \Lambda^{\operatorname{dim} V_{v}-i} V_{v,-n+(p-1) v+\frac{p_{j} v}{n_{j}}+\frac{1}{2}} \otimes \operatorname{det} \bar{V}_{v} .
$$

From (3.6), (4.33), as in (3.9), we get the last equality of (4.28). The proof of Proposition 4.1 is complete. 
Lemma 4.3. Let us introduce the following two line bundles :

$$
\begin{aligned}
L\left(\beta_{j}\right)_{1}= & L_{1}^{\prime} \otimes \bigotimes_{0<\imath}\left(\operatorname{det} N_{v}\right)^{\left[\frac{p_{j} v}{n_{j}}\right]+(p-1) v+1} \\
& \otimes \bigotimes_{0<v}\left(\operatorname{det} \bar{V}_{v}\right)^{\left[\frac{p_{j} v}{n_{j}}\right]+(p-1) v} \otimes \bigotimes_{\substack{0<v, v=0 \bmod \left(n_{j}\right)}}\left(\operatorname{det} N_{v}\right)^{-1}, \\
L\left(\beta_{j}\right)_{2}= & L_{2}^{\prime} \otimes \bigotimes_{0<v}\left(\operatorname{det} N_{v}\right)^{\left[\frac{p_{j} v}{n_{j}}\right]+(p-1) v+1} \\
& \otimes \bigotimes_{0<v}\left(\operatorname{det} \bar{V}_{v}\right)^{\left[\frac{p_{j} v}{n_{j}}+\frac{1}{2}\right]+(p-1) v} \otimes \bigotimes_{\substack{0<v, \bmod \left(n_{j}\right)}}\left(\operatorname{det} N_{v}\right)^{-1} .
\end{aligned}
$$

Then $L\left(\beta_{j}\right)_{1}, L\left(\beta_{j}\right)_{2}$ can be extended naturally to $S^{1}$-equivariant complex line bundles which we will still denote by $L\left(\beta_{j}\right)_{1}, L\left(\beta_{j}\right)_{2}$ respectively over $M\left(n_{j}\right)$.

Proof: We divide the argument into several steps.

1) Write

$$
\left[\frac{p_{j} v}{n_{j}}\right]=\frac{p_{j} v}{n_{j}}-\frac{\omega(v)}{n_{j}} .
$$

Note that for $v=\frac{n_{j}}{2} \bmod \left(n_{j}\right), \frac{\omega(v)}{n_{j}}=\frac{1}{2}$. From (2.10), (4.19) and (4.35), we get, formally,

$$
\begin{aligned}
& \text { (4.36) } L\left(\beta_{j}\right)_{1}=L^{-(p-1)-p_{j} / n_{j}} \otimes \quad \bigotimes \quad\left(\operatorname{det} N_{v} \otimes \operatorname{det} \bar{V}_{v}\right)^{-\frac{\omega(v)}{n_{j}}} \\
& 0<v, v \neq \frac{n_{j}}{2} \bmod \left(n_{j}\right) \\
& \otimes \bigotimes_{0<v, \frac{n_{j}}{i}<<<n_{j} \bmod \left(n_{j}\right)}\left(\operatorname{det} N_{v} \otimes \operatorname{det} \bar{V}_{v}\right) \otimes L\left(n_{j}\right)^{\frac{-r\left(n_{j}\right)}{2 n_{j}}} .
\end{aligned}
$$

Note that, if $v=v^{\prime} \bmod \left(n_{j}\right)$, then $\omega(v)=\omega\left(v^{\prime}\right)$. Also, for $0<v^{\prime}<n_{j}$, $\omega\left(n_{j}-v^{\prime}\right)=n_{j}-\omega\left(v^{\prime}\right)$. From (4.6), (4.10), (4.11) and (4.36), formally, we get

$$
\begin{aligned}
L\left(\beta_{j}\right)_{1} & =L^{-(p-1)-p_{j} / n_{j}} \\
& \otimes\left[\bigotimes_{0<v<\frac{n_{j}}{2}}\left(\operatorname{det}\left(N\left(n_{j}\right)_{v}\right) \otimes \operatorname{det}\left(\overline{V\left(n_{j}\right)_{v}}\right)\right)^{-\omega(v)-r\left(n_{j}\right) v}\right]^{1 / n_{j}} .
\end{aligned}
$$


We introduce the following line bundle over $M\left(n_{j}\right)$,

$$
L^{\omega}\left(\beta_{j}\right)=\bigotimes_{0<v<\frac{n_{j}}{2}}\left(\operatorname{det}\left(N\left(n_{j}\right)_{v}\right) \otimes \operatorname{det}\left(\overline{V\left(n_{j}\right)_{v}}\right)\right)^{-\omega(v)-r\left(n_{j}\right) v} .
$$

Note that $\omega(v)=p_{j} v \bmod \left(n_{j}\right)$. If $n_{j}$ is odd, then $r\left(n_{j}\right)=0$, Lemma 4.2 implies that $L^{\omega}\left(\beta_{j}\right)^{1 / n_{j}}$ is well defined over $M\left(n_{j}\right)$. If $n_{j}$ is even, then $p_{j}$ is odd, and $r\left(n_{j}\right)=1$, Lemma 4.2 again implies $L^{\omega}\left(\beta_{j}\right)^{1 / n_{j}}$ is well defined over $M\left(n_{j}\right)$. So $L\left(\beta_{j}\right)_{1}$ extends naturally over $M\left(n_{j}\right)$.

2) From (2.10), (4.19), (4.34), we get

$$
L\left(\beta_{j}\right)_{2}=L^{-(p-1)} \otimes \bigotimes_{0<v}\left(\operatorname{det} N_{v}\right)^{\left[\frac{p_{j} v}{n_{j}}\right]} \otimes \bigotimes_{0<v}\left(\operatorname{det} \bar{V}_{v}\right)^{\left[\frac{p_{j} v}{n_{j}}+\frac{1}{2}\right]}
$$

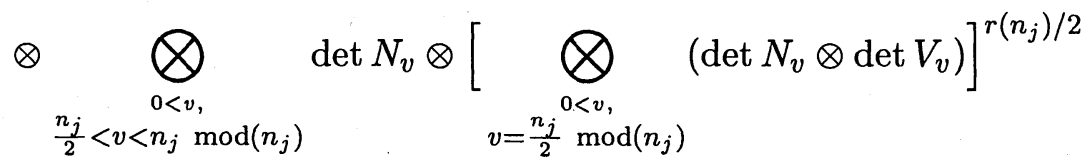

$$
\otimes L\left(n_{j}\right)^{-r\left(n_{j}\right) / 2 n_{j}}
$$

By using the same argument as in 1), one deduces that

$$
\begin{aligned}
& \bigotimes_{0<v}\left(\operatorname{det} N_{v}\right)^{-\omega(v) / n_{j}} \otimes \bigotimes_{\substack{0<v, \frac{n_{j}}{2}<v<n_{j} \bmod \left(n_{j}\right)}} \operatorname{det} N_{v} \otimes[\underbrace{}_{\substack{v=\frac{n_{j}}{2} \bmod \left(n_{j}\right)\\
}} \operatorname{det} N_{v}]^{r\left(n_{j}\right) / 2} \\
&=\bigotimes_{0<v<\frac{n_{j}}{2}}\left(\operatorname{det}\left(N\left(n_{j}\right) v\right)\right)^{-\omega(v) / n_{j}} .
\end{aligned}
$$

Note that, if there exists $m \in\left[0, p_{j}\left[\right.\right.$ with $m \in \mathbf{N}$, such that $m \leq p_{j} v / n_{j}<$ $\left(m+\frac{1}{2}\right)$, then

$$
\left[\frac{p_{j} v}{n_{j}}+\frac{1}{2}\right]=p_{j} v / n_{j}-\omega(v) / n_{j}
$$

While if there exists $\left.m \in] 0, p_{j}\right], m \in \mathbf{N}$ such that $\left(m-\frac{1}{2}\right) \leq p_{j} v / n_{j}<m$, then

$$
\left[\frac{p_{j} v}{n_{j}}+\frac{1}{2}\right]=p_{j} v / n_{j}+\left(n_{j}-\omega(v)\right) / n_{j}
$$


From (4.11), we have the following formal identity,

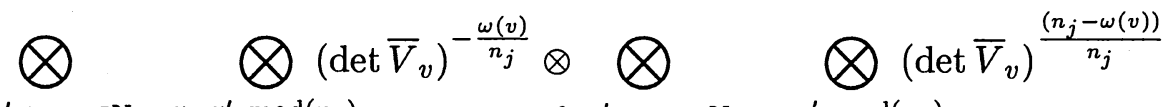

$$
\begin{aligned}
& \begin{array}{c}
0<v^{\prime}<n_{j}, m \in \mathbf{N} \\
m<p_{j} v^{\prime} / n_{j}<m+\frac{1}{2}
\end{array} \\
& =\bigotimes_{0<v^{\prime}<n_{j} / 2, m \in \mathbf{N}}\left(\bigotimes_{v=v^{\prime} \bmod \left(n_{j}\right)}\left(\operatorname{det} \bar{V}_{v}\right)^{-\frac{\omega\left(v^{\prime}\right)}{n_{j}}} \otimes \bigotimes_{v=-v^{\prime} \bmod \left(n_{j}\right)}\left(\operatorname{det} \bar{V}_{v}\right)^{\frac{\omega\left(v^{\prime}\right)}{n_{j}}}\right) \\
& m<p_{j} v^{\prime} / n_{j}<m+\frac{1}{2} \\
& \otimes \bigotimes_{\substack{0<v^{\prime}<n_{j} / 2, m \in \mathbf{N} \\
m-\frac{1}{2}<p_{j} v^{\prime} / n_{j}<m}}\left(\bigotimes_{v=-v^{\prime} \bmod \left(n_{j}\right)}\left(\operatorname{det} \bar{V}_{v}\right)^{-\frac{\left(n_{j}-\omega\left(v^{\prime}\right)\right)}{n_{j}}} \otimes \bigotimes_{\substack{v=v^{\prime} \\
\bmod \left(n_{j}\right)}}\left(\operatorname{det} \bar{V}_{v}\right)^{\frac{\left(n_{j}-\omega\left(v^{\prime}\right)\right)}{n_{j}}}\right) \\
& =\bigotimes_{0<v^{\prime}<n_{j} / 2}\left(\operatorname{det}\left(\overline{V\left(n_{j}\right)_{v^{\prime}}}\right)\right)^{-\frac{\omega\left(v^{\prime}\right)}{n_{j}}} \otimes \bigotimes_{1 \leq m \leq \frac{p_{j}}{2} m-\frac{1}{2}<p_{j} v^{\prime} / n_{j}<m} \operatorname{det}\left(\overline{V\left(n_{j}\right)_{v^{\prime}}}\right) \text {. }
\end{aligned}
$$

Recall that for $v \in \mathbf{Z}, p_{j} v / n_{j} \in \mathbf{Z}$ iff $v / n_{j} \in \mathbf{Z}$, also $p_{j} v / n_{j} \in \mathbf{Z}+\frac{1}{2}$ iff $v / n_{j} \in \mathbf{Z}+\frac{1}{2}$. From (2.10), (4.39)-(4.43), we get

$$
L\left(\beta_{j}\right)_{2}=L^{-(p-1)-\frac{p_{j}}{n_{j}}} \otimes L^{\omega^{\prime}}\left(\beta_{j}\right)^{\frac{1}{n_{j}}} \otimes \bigotimes_{1 \leq m \leq \frac{p_{j}}{2} m-\frac{1}{2}<p_{j} v^{\prime} / n_{j}<m} \operatorname{det}\left(\overline{V\left(n_{j}\right)_{v^{\prime}}}\right) .
$$

The proof of Lemma 4.3 is complete.

Let us denote by

$$
\begin{gathered}
\varepsilon_{1}=\frac{1}{2} \sum_{0<v}\left(\operatorname{dim} N_{v}-\operatorname{dim} V_{v}\right)\left[\left(\left[\frac{p_{j} v}{n_{j}}\right]+(p-1) v\right)\left(\left[\frac{p_{j} v}{n_{j}}\right]+(p-1) v+1\right)\right. \\
\left.-\left(\frac{p_{j} v}{n_{j}}+(p-1) v\right)\left(2\left(\left[\frac{p_{j} v}{n_{j}}\right]+(p-1) v\right)+1\right)\right],
\end{gathered}
$$




$$
\begin{gathered}
\varepsilon_{2}=\frac{1}{2} \sum_{0<v}\left(\operatorname{dim} N_{v}\right)\left[\left(\left[\frac{p_{j} v}{n_{j}}\right]+(p-1) v\right)\left(\left[\frac{p_{j} v}{n_{j}}\right]+(p-1) v+1\right)\right. \\
\left.-\left(\frac{p_{j} v}{n_{j}}+(p-1) v\right)\left(2\left(\left[\frac{p_{j} v}{n_{j}}\right]+(p-1) v\right)+1\right)\right] \\
-\frac{1}{2} \sum_{0<v}\left(\operatorname{dim} V_{v}\right)\left[\left(\left[\frac{p_{j} v}{n_{j}}+\frac{1}{2}\right]+(p-1) v\right)^{2}\right. \\
\left.-2\left(\frac{p_{j} v}{n_{j}}+(p-1) v\right)\left(\left[\frac{p_{j} v}{n_{j}}+\frac{1}{2}\right]+(p-1) v\right)\right] .
\end{gathered}
$$

Then $\varepsilon_{1}, \varepsilon_{2}$ are locally constant functions on $F$.

Recall that, if $E$ is a $S^{1}$-equivariant vector bundle over $M$, then the weight of the $S^{1}$ action on $\Gamma(F, E)$ is given by the action $\mathbf{J}_{H}$ (cf. Section $3.1)$.

Proposition 4.2. For $i=1,2$, the isomorphisms induced by (4.20) and (4.28):

$r_{i 1}: S\left(T Y, \otimes_{0<v}\left(\operatorname{det} N_{v}\right)^{-1}\right) \otimes \mathcal{F}_{p, j-1}(X) \otimes F_{V}^{i} \rightarrow$

$$
S\left(U_{i}, L_{i}\right)^{\prime} \otimes \mathcal{F}\left(\beta_{j}\right) \otimes F_{V}^{i}\left(\beta_{j}\right) \otimes L\left(\beta_{j}\right)_{i} \otimes \bigotimes_{\substack{0<v_{j} \\ v=0 \bmod \left(n_{j}\right)}} \operatorname{Sym}\left(\bar{N}_{v, 0}\right),
$$

$r_{i 2}: S\left(T Y, \otimes_{0<v}\left(\operatorname{det} N_{v}\right)^{-1}\right) \otimes \mathcal{F}_{p, j}(X) \otimes F_{V}^{i} \rightarrow$

$$
\begin{aligned}
S\left(U_{i}, L_{i}\right)^{\prime} \otimes \mathcal{F}\left(\beta_{j}\right) \otimes F_{V}^{i}\left(\beta_{j}\right) \otimes L\left(\beta_{j}\right)_{i} & \otimes \bigotimes_{\substack{0<v, j \\
v=0 \bmod \left(n_{j}\right)}}\left(\operatorname{Sym}\left(N_{v, 0}\right) \otimes \operatorname{det} N_{v}\right),
\end{aligned}
$$

have the following properties :

1) for $i=1,2 ; \gamma=1,2$, one has

$$
\begin{aligned}
& r_{i \gamma}^{-1} \mathbf{J}_{H} r_{i \gamma}=\mathbf{J}_{H}, \\
& r_{i \gamma}^{-1} P r_{i \gamma}=P+\left(\frac{p_{j}}{n_{j}}+(p-1)\right) \mathbf{J}_{H}+\varepsilon_{i \gamma},
\end{aligned}
$$

where

$$
\varepsilon_{i 1}=\varepsilon_{i}-e\left(p, \beta_{j-1}, N\right), \quad \varepsilon_{i 2}=\varepsilon_{i}-e\left(p, \beta_{j}, N\right) .
$$


2) Recall that $o\left(V\left(n_{j}\right)_{\frac{n_{j}}{2}}^{\mathbf{R}}\right)$ was defined in (4.25). Denote by (4.49) $\mu_{1}=-\sum_{0<v}\left[\frac{p_{j} v}{n_{j}}\right] \operatorname{dim} V_{v}+\Delta\left(n_{j}, N\right)+\Delta\left(n_{j}, V\right) \bmod (2)$,

$$
\begin{aligned}
& \mu_{2}=-\sum_{0<v}\left[\frac{p_{j} v}{n_{j}}+\frac{1}{2}\right] \operatorname{dim} V_{v}+\Delta\left(n_{j}, N\right)+o\left(V\left(n_{j}\right)_{\frac{n_{j}}{2}}^{\mathbf{R}}\right) \bmod (2), \\
& \mu_{3}=\Delta\left(n_{j}, N\right) \bmod (2) .
\end{aligned}
$$

Then, for $i=1,2, \gamma=1,2$, one has

$$
r_{i \gamma}^{-1} \tau_{e} r_{i \gamma}=(-1)^{\mu_{i}} \tau_{e}, \quad r_{i \gamma}^{-1} \tau_{s} r_{i \gamma}=(-1)^{\mu_{3}} \tau_{s}
$$

Proof: The first equality of (4.47) is trivial.

- From (2.24) and (4.30), one has

(4.51) $e\left(p, \beta_{j}, N\right)=e\left(p, \beta_{j-1}, N\right)+\sum_{0<v, v=0 \bmod \left(n_{j}\right)}\left((p-1) v+\frac{p_{j} v}{n_{j}}\right) \operatorname{dim} N_{v}$.

For $i=1,2$, denote by $\varepsilon_{i}(V)$ the contribution of $\operatorname{dim} V$ to $\varepsilon_{i}$. From (4.32), when acting on $\bigotimes_{\substack{0<v, n \in \mathbf{N} \\ 0<n \leq(p-1) v+\frac{p_{j} v}{n_{j}}}} \Lambda^{i_{n}} \bar{V}_{v, n}$, as in (3.20), we get that

$$
\begin{aligned}
r_{j *}^{-1} P r_{j *} & =P+\left((p-1)+\frac{p_{j}}{n_{j}}\right) \mathbf{J}_{H} \\
& +\sum_{\substack{0<v, n \in \mathbb{N}, 0<n \leq(p-1) v+\frac{p_{j} v}{n_{j}}}}\left(\operatorname{dim} V_{v}\right)\left(-n+(p-1) v+\frac{p_{j} v}{n_{j}}\right) \\
& =P+\left((p-1)+\frac{p_{j}}{n_{j}}\right) \mathbf{J}_{H}+\varepsilon_{1}(V)-\frac{1}{2}\left((p-1)+\frac{p_{j}}{n_{j}}\right) d^{\prime}(V) .
\end{aligned}
$$

Similarly, by (4.33), when acting on $\otimes_{\substack{0<n \leq(p-1) v+\frac{p_{j^{v}}}{n_{j}}+\frac{1}{2} \\ 0<n}} \Lambda^{i_{n}} \bar{V}_{v, n-\frac{1}{2}}$, as in (3.24), we get that

$$
\begin{aligned}
r_{j *}^{-1} P r_{j *}=P+\left((p-1)+\frac{p_{j}}{n_{j}}\right) & \mathbf{J}_{H} \\
+\sum_{\substack{0<v, n \in \mathbf{N}_{,}, 0<n \leq(p-1) v+\frac{p_{j} v}{n_{j}}+\frac{1}{2}}}\left(\operatorname{dim} V_{v}\right) & \left(-n+(p-1) v+\frac{p_{j} v}{n_{j}}+\frac{1}{2}\right) \\
& =P+\left((p-1)+\frac{p_{j}}{n_{j}}\right) \mathbf{J}_{H}+\varepsilon_{2}(V) .
\end{aligned}
$$


From (2.24), (3.21), (4.29)-(4.31), (4.51), (4.52), (4.53), and by proceeding as in the proof of Proposition 3.2, one deduces easily the second equation of (4.47).

- From (4.32), on $\bigotimes_{\substack{0<v, n \in \mathbf{N} \\ 0<n \leq(p-1) v+\frac{p_{j} v}{n_{j}}}} \Lambda^{i_{n}} \bar{V}_{v, n}$, we have

$$
r_{j *}^{-1} \tau_{e} r_{j *}=(-1)^{-\sum_{0<v}\left(\left[\frac{p_{j} v}{n_{j}}\right]+(p-1) v\right) \operatorname{dim} V_{v}} \tau_{e}
$$

From (4.33), on $\bigotimes_{\substack{0<v, n \in \mathbf{N}_{p_{j}} \\ 0<n \leq(p-1) v+\frac{p^{\prime}}{n_{j}}+\frac{1}{2}}} \Lambda^{i_{n}} \bar{V}_{v, n-\frac{1}{2}}$, we have

$$
r_{j *}^{-1} \tau_{e} r_{j *}=(-1)^{-\sum_{0<v}\left(\left[\frac{p_{j} v}{n_{j}}+\frac{1}{2}\right]+(p-1) v\right) \operatorname{dim} V_{v}} \tau_{e} .
$$

As we don't change the $\mathbf{Z}_{2}$-grading in the rest part of (4.46), from (3.30), (4.17), (4.54), (4.55) and the discussion following (4.25), we get (4.50). The proof of Proposition 4.2 is complete.

The following Lemmas 4.4 and 4.6 were essentially proved in [T, Lemmas $9.6,9.7]$.

Lemma 4.4. For each connected component $M^{\prime}$ of $M\left(n_{j}\right), \varepsilon_{1}, \varepsilon_{2}$ are independent on the connected component of $F$ in $M^{\prime}$.

Proof: From (2.12), (4.11), (4.13), (4.35) and (4.45), we get

$$
\begin{gathered}
\varepsilon_{1}=\frac{1}{2} \sum_{0 \leq v^{\prime}<n_{j}} \sum_{0<v, v=v^{\prime} \bmod \left(n_{j}\right)}\left(\operatorname{dim} N_{v}-\operatorname{dim} V_{v}\right)\left[-\left(\frac{p_{j} v}{n_{j}}+(p-1) v\right)^{2}\right. \\
\left.-\frac{\omega\left(v^{\prime}\right)\left(n_{j}-\omega\left(v^{\prime}\right)\right)}{n_{j}^{2}}\right] \\
=\left(p-1+\frac{p_{j}}{n_{j}}\right)^{2} e-\frac{1}{16}\left(\operatorname{dim}_{\mathbf{R}} N\left(n_{j}\right)_{\frac{n_{j}}{2}}^{\mathbf{R}}-\operatorname{dim}_{\mathbf{R}} V\left(n_{j}\right)_{\frac{n_{j}}{2}}^{\mathbf{R}}\right) \\
\quad-\frac{1}{2} \sum_{0<v^{\prime}<n_{j} / 2}\left(\operatorname{dim} N\left(n_{j}\right)_{v^{\prime}}-\operatorname{dim} V\left(n_{j}\right)_{v^{\prime}}\right) \frac{\omega\left(v^{\prime}\right)\left(n_{j}-\omega\left(v^{\prime}\right)\right)}{n_{j}^{2}} .
\end{gathered}
$$


By combining (4.11), (4.13), (4.16), (4.41), (4.42) and (4.45), as in (4.56), we get

$$
\begin{aligned}
& \text { (4.57) } \varepsilon_{2}=\varepsilon_{1}-\frac{1}{2} \sum_{0 \leq m<p_{p^{i}}} \sum_{m<p_{j} v^{\prime} / n_{j}<m+\frac{1}{2}}\left(\operatorname{dim} V\left(n_{j}\right)_{v^{\prime}}\right) \frac{\omega\left(v^{\prime}\right)}{n_{j}} \\
& -\frac{1}{2} \sum_{0<m \leq p_{j}} \sum_{m-\frac{1}{2}<p_{j} v^{\prime} / n_{j}<n_{i}}\left(\operatorname{dim} V\left(n_{j}\right)_{v^{\prime}}\right) \frac{\left(n_{j}-\omega\left(v^{\prime}\right)\right)}{n_{j}}-\frac{1}{8} \operatorname{dim}_{\mathbf{R}} V\left(n_{j}\right)_{\frac{n_{j}}{2}}^{\mathbf{R}} .
\end{aligned}
$$

The proof of Lemma 4.4 is complete.

The following Lemma 'was proved in [BT, Lemma 9.3].

Lemma 4.5. Let $M$ be a smooth manifold on which $S^{1}$ acts. Let $M^{\prime}$ be a connected component of $M\left(n_{j}\right)$, the fixed point set of $\mathbf{Z}_{n_{j}}$ subgroup of $S^{1}$.on $M$. Let $F$ be the fixed point set of $S^{1}$-action on $M$. Let $V \rightarrow M$ be a real, oriented, even dimensionail vector bundle to which the $S^{1}$-action on $M$ lifts. Assume that $V$ is spin over $M$. Let $\left.p_{j} \in\right] 0, n_{j}\left[, p_{j} \in \mathbf{N}\right.$ and $\left(p_{j}, n_{j}\right)=1$, then

$$
\sum_{0<v}\left(\operatorname{dim} V_{v}\right)\left[\frac{p_{j} v}{n_{j}}\right]+\Delta\left(n_{j}, V\right) \bmod (2)
$$

is independent on the connected components of $F$ in $M^{\prime}$.

Recall that the number $d^{\prime}\left(\beta_{j}, N\right)$ has been defined in (2.24).

Lemma 4.6. For each connected component $M^{\prime}$ of $M\left(n_{j}\right), d^{\prime}\left(\beta_{j}, N\right)+$ $\mu_{i} \bmod (2)(i=1,2,3)$ is independent on the connected component of $F$ in $M^{\prime}$.

Proof: The assertions for the cases $i=1,3$ follow immediately from Lemma 4.5 by replacing $V$ with $T X$. From (4.41) and (4.42), we have

$$
\begin{gathered}
o\left(V\left(n_{j}\right)_{n_{j} / 2}^{\mathbf{R}}\right)+\sum_{0<v}\left(\operatorname{dim} V_{\imath}\right)\left[\frac{p_{j} v}{n_{j}}+\frac{1}{2}\right]=\sum_{0<v}\left(\operatorname{dim} V_{v}\right)\left[\frac{p_{j} v}{n_{j}}\right]+o\left(V\left(n_{j}\right)_{n_{j} / 2}^{\mathbf{R}}\right) \\
+\sum_{\substack { 0<m \leq p_{j} \\
\begin{subarray}{c}{0<v^{\prime}<r_{j}, m-\frac{1}{2}<p_{j} v^{\prime} i_{j}<m{ 0 < m \leq p _ { j } \\
\begin{subarray} { c } { 0 < v ^ { \prime } < r _ { j } , \\
m - \frac { 1 } { 2 } < p _ { j } v ^ { \prime } i _ { j } < m } }\end{subarray}} \sum_{\substack{0<v, v=v^{\prime} \bmod \left(n_{j}\right)}} \operatorname{dim} V_{v}+\sum_{\substack{0<v \\
v=\frac{n_{j}}{2} \bmod \left(n_{j}\right)}} \operatorname{dim} V_{v} .
\end{gathered}
$$


By (4.13), the last term in (4.59), $\operatorname{dim}_{\mathbf{R}} V\left(n_{j}\right)_{n_{j} / 2}^{\mathbf{R}}$, is a locally constant function on $M\left(n_{j}\right)$.

By (4.11), the third term on the right side of (4.59) is $\bmod (2)$ equal to (4.60)

$$
\sum_{n_{j} / 2<v^{\prime}<n_{j}} \sum_{0<v, v=v^{\prime} \bmod \left(n_{j}\right)} \operatorname{dim} V_{v}+\sum_{0<m \leq p_{j}} \sum_{\substack{0<v^{\prime}<n_{j} / 2 \\ m-\frac{1}{2}<p_{j} / n_{j}<m}} \operatorname{dim} V\left(n_{j}\right)_{v^{\prime}}
$$

The last term of (4.60) is a locally constant function on $M\left(n_{j}\right)$.

By (4.25), the first term in (4.60) is $o\left(V\left(n_{j}\right)_{n_{j} / 2}^{\mathbf{R}}\right)+\Delta\left(n_{j}, V\right) \bmod (2)$. On the other hand, by Lemma 4.5 , we know that $\sum_{0<v}\left(\operatorname{dim} V_{v}\right)\left[\frac{p_{j} v}{n_{j}}\right]+$ $\Delta\left(n_{j}, V\right) \bmod (2)$ is independent on the component of $F$ in $M^{\prime}$.

By the above discussion, the left side of (4.59) is $\bmod (2)$ independent on the component of $F$ in $M^{\prime}$. The proof of Lemma 4.6 is complete.

\subsection{Proof of Theorem 2.7.}

From (2.24), (4.10), (4.13) and (4.30), we have

$$
\begin{aligned}
& \sum_{0<v} \operatorname{dim} N_{v}=\sum_{0<v<\frac{n_{j}}{2}} \operatorname{dim} N\left(n_{j}\right)_{v}+\frac{1}{2} \operatorname{dim} \mathbf{R} N\left(n_{j}\right)_{n_{j} / 2}^{\mathbf{R}}+\sum_{0<v, v=0 \bmod \left(n_{j}\right)} \operatorname{dim} N_{v}, \\
& d^{\prime}\left(\beta_{j}, N\right)=d^{\prime}\left(\beta_{j-1}, N\right)+\sum_{0<v, v=0 \bmod \left(n_{j}\right)} \operatorname{dim} N_{v} .
\end{aligned}
$$

By Lemma 4.6, and (4.61), $d^{\prime}\left(\beta_{j-1}, N\right)+\sum_{0<v} \operatorname{dim} N_{v}+\mu_{i} \bmod (2)$ is a constant function on each connected component of $M\left(n_{j}\right)$.

From Lemma 4.3, one knows that the Dirac operator $D^{X\left(n_{j}\right)} \otimes F\left(\beta_{j}\right) \otimes$ $F_{V}^{i}\left(\beta_{j}\right) \otimes L\left(\beta_{j}\right)_{i}(i=1,2)$ is well-defined on $M\left(n_{j}\right)$. Thus, by using Proposition 4.2, Lemma 4.4, (4.9), (4.18) and (4.61), for $i=1,2, h \in \mathbf{Z}, m \in \frac{1}{2} \mathbf{Z}$, $\tau=\tau_{e}$ or $\tau_{s}$, and by applying separately both the first and the second equations of Theorem 1.2 to each connected component of $M\left(n_{j}\right)$, we get the 
following identity in $K(B)$,

$$
\begin{aligned}
& \text { (4.62) } \quad \sum_{\alpha}(-1)^{d^{\prime}\left(\beta_{j-1}, N\right)+\sum_{0<v} \operatorname{dim} N_{v}} \operatorname{Ind}_{\tau}\left(D^{Y_{\alpha}} \otimes \mathcal{F}_{p, j-1}(X) \otimes F_{V}^{i},\right. \\
& \left.m+e\left(p, \beta_{j-1}, N\right), h\right) \\
& =\sum_{\beta}(-1)^{d^{\prime}\left(\beta_{j-1}, N\right)+\sum_{0<v} \operatorname{dim} N_{v}+\mu} \operatorname{Ind}_{\tau}\left(D^{X\left(n_{j}\right)} \otimes F\left(\beta_{j}\right) \otimes F_{V}^{i}\left(\beta_{j}\right) \otimes L\left(\beta_{j}\right)_{i},\right. \\
& \left.m+\varepsilon_{i}+\left(\frac{p_{j}}{n_{j}}+(p-1)\right) h, h\right) \\
& =\sum_{\alpha}(-1)^{d^{\prime}\left(\beta_{j}, N\right)+\sum_{0<v} \operatorname{din} N_{v}} \operatorname{Ind}_{\tau}\left(D^{Y_{\alpha}} \otimes \mathcal{F}_{p, j}(X) \otimes F_{V}^{i}, m+e\left(p, \beta_{j}, N\right), h\right) \text {. }
\end{aligned}
$$

Here $\sum_{\beta}$ means the sum over all the connected components of $M\left(n_{j}\right)$. In (4.62), if $\tau=\tau_{s}$, then $\mu=u_{3}$; if $\tau=\tau_{e}$, then $\mu=\mu_{i}$. The proof of Theorem 2.7 is complete.

\section{References.}

[AH] Atiyah M.F. and Hirzebruch F., Spin manifolds and groups actions, Essays on topolog! and Related Topics, Memoires dédié à Georges de Rham (ed. A. Haefliger and R. Narasimhan), Springer-Verlag, New York-Berlin (1970), 18-28.

[ASe] Atiyah M.F. and Segal G., The index of elliptic operators II, Ann. of Math.87 (1968), 531-545.

[ASi] Atiyah M.F., Singer I.M., The index of elliptic operators III, Ann. of Math. 87 (1968), 546-604.

[AS] Atiyah M.F. and Singer I.M., The index of elliptic operators IV, Ann. of Math. 93 (1971), 119-138.

[BL] Bismut J.-M. and Jebeau G., Complex immersions and Quillen metrics, Publ. Math. IHES. 74 (1991), 1-297.

[BT] Bott R. and Taubes C., On the rigidity theorems of Witten, J.A.M.S. 2 (1989), 137-186.

[De] Dessai A., The Wirten genus and $S^{3}$-actions on manifolds, Preprint, 1994. 
[DeJ] Dessai A. and Jung R., On the rigidity theorem for elliptic genera, Trans. A.M.S. 350 (1998), 4195-4220.

[E] Edmonds A.L., Orientability of fixed point sets, Proc. Amer. Math. Soc. 82 (1981), 120-124.

[H] F. Hirzebruch, Elliptic genera of level $N$ for complex manifolds, in Differential Geometric Methods in Theoretic Physics. Kluwer, Dordrecht, 1988, pp. 37-63.

[K] Krichever, I., Generalized elliptic genera and Baker-Akhiezer functions, Math. Notes 47 (1990), 132-142.

[L] Landweber P.S. (ed.), Elliptic Curves and Modular forms in Algebraic Topology (Princeton 1986), LNM 1326, Springer, Berlin, 1988.

[LS] Landweber P.S. and Stong R.E., Circle actions on spin manifolds and characteristic numbers, Topology. 27 (1988), 145-161.

[LaM] Lawson H.B. and Michelsohn M.L., Spin Geometry, Princeton Univ. Press, Princeton, 1989.

[Liu1] Liu K., On elliptic genera and theta-functions, Topology 35 (1996), 617-640.

[Liu2] Liu K., On modular invariance and rigidity theorems, J. Diff. Geom. 41 (1995), 343-396.

[LM1] Liu K. and Ma X., On family rigidity theorems I, Duke Math. J. $102(2000), 451-474$.

[LM2] Liu K. and Ma X., On family rigidity theorems for $\operatorname{Spin}^{c}$ Manifolds, Mirror Symmetry 4, AMS/IP Studies in Advanced Mathematics Vol. 28 (2002).

[LMZ1] Liu K., Ma X. and Zhang W., Rigidity and Vanishing Theorems in K-Theory, C. R. Acad. Sci. Paris, Série A 330 (2000), 301-306.

[LMZ2] Liu K., Ma X. and Zhang W., Spin ${ }^{c}$ Manifolds and Rigidity Theorems in K-Theory, Asian J. Math. 4 (2000), 933-960.

[O] Ochanine S., Sur les genres multiplicatifs définis par des intégrales elliptiques. Topology. 26 (1987), 143-151. 
[S] Segal G., Equivariant K-Theory, Publ. Math. IHES. 34 (1968), 129151.

[T] Taubes C., $S^{1}$ actions and elliptic genera, Comm. Math. Phys. 122 (1989), 455-526.

[W] Witten E., The index of the Dirac operator in loop space, in [L], 161-186.

[WuZ] Wu S. and Zhang W., Equivariant holomorphic Morse inequalities III: non-isolated fixed points, Geom. Funct. Anal. 8 (1998) 149-178.

[Z] Zhang W., Symplectic reduction and family quantization, Inter. Math. Res. Notices No.19, (1999), 1043-1055.

Department of Mathema'TiCS

UNIVERSITY OF CALIFORNIA. AT LOS ANGELES

CA 90095-1555

USA

E-mail address: liu@math.ucla.edu

CNRS UMR7640, Centre de MathÉmatiques

Ecole Polytechnique

91128 Palaiseau Cedex

FRANCE

E-mail address: ma@math.polytechnique.fr

Nankai Institute of Mathematics

NANKAI UNIVERSITY

TIANJIN 300071

People's Republic of China

E-mail address: weiping@na.zkai.edu.cn

RECEIVED JANUARY 22, 20012. 